\title{
Hole-hole Tamm-Dancoff-approximated density functional theory: a highly efficient electronic structure method incorporating dynamic and static correlation
}

Christoph Bannwarth, ${ }^{1,2}$ Jimmy K. Yu, ${ }^{1,2,3}$ Edward G. Hohenstein, ${ }^{1,2}$ and Todd J. Martínez,2,**

${ }^{1}$ Department of Chemistry and The PULSE Institute, Stanford University, Stanford, CA 94305

${ }^{2}$ SLAC National Accelerator Laboratory, 2575 Sand Hill Road, Menlo Park, CA 94025

${ }^{3}$ Biophysics Program, Stanford University, Stanford, CA 94305

*toddjmartinez@gmail.com

\begin{abstract}
The study of photochemical reaction dynamics requires accurate as well as computationally efficient electronic structure methods for the ground and excited states. While time-dependent density functional theory (TDDFT) is not able to capture static correlation, complete active space self-consistent field (CASSCF) methods neglect much of the dynamic correlation. Hence, inexpensive methods that encompass both static and dynamic electron correlation effects are of high interest. Here, we revisit hole-hole Tamm-Dancoff approximated ( $h h$-TDA) density functional theory for this purpose. The $h h$-TDA method is the hole-hole counterpart to the more established particle-particle TDA ( $p p$-TDA) method, both of which are derived from the particle-particle random phase approximation ( $p p$-RPA). In $h h$-TDA, the $N$ electron electronic states are obtained through double annihilations starting from a doubly anionic $(N+2$ electron) reference state. In this way, $h h$-TDA treats ground and excited states on equal footing, thus allowing for conical intersections to be correctly described. The treatment of dynamic correlation is introduced through the use of commonly-employed density functional approximations to the exchange-correlation potential. We show that $h h$-TDA is a promising candidate to efficiently treat the photochemistry of organic and biochemical systems that involve several low-lying excited states - particularly those with both low-lying $\pi \pi^{*}$ and $n \pi^{*}$ states where inclusion of dynamic correlation is essential to describe the relative energetics. In contrast to the existing literature on $p p$-TDA and $p p$-RPA, we employ a functional-dependent choice for the response kernel in $p p$ - and $h h$-TDA, which closely resembles the response kernels occurring in linear response and collinear spin-flip TDDFT.
\end{abstract}

Bannwarth, Yu, Hohenstein, Martínez - hh-TDA - Page 1 


\section{Introduction}

Linear response time-dependent density functional theory (TDDFT) is the most commonly used electronic structure method for excited states due to its low computational cost and relative accuracy for absorption spectra involving valence excited states. ${ }^{1}$ Unfortunately, due to the difficulties that presently available approximate exchange-correlation functionals encounter with near-degeneracies and static correlation, it is unsuitable for photochemical problems involving a conical intersection between the ground and first excited states. ${ }^{2}$ While complete active space selfconsistent field (CASSCF) methods can treat static electron correlation, they struggle to describe dynamic correlation. ${ }^{3}$ Adding a correction to recover dynamic correlation (as in multireference perturbation theory ${ }^{4}$ or multireference configuration interaction ${ }^{5}$ ) significantly increases the computational cost of the method and renders nonadiabatic dynamics simulations computationally intractable for many interesting medium to large sized molecules (although such dynamics is feasible for small molecules ${ }^{6-12}$ ).

There has long been interest in the development of inexpensive excited-state methods that can simultaneously treat dynamic and static correlation. One approach to this problem is to augment traditional multiconfigurational methods with semiempirical parameters. These parameters can be as simple as a constant scaling parameter applied to the energy ${ }^{13}$ or as complex as a full semiempirical treatment of the Hamiltonian matrix elements. ${ }^{14-15}$ While often successful, these methods may require cumbersome system-specific parameterization. ${ }^{15-17}$ Alternatively, conventional multiconfigurational wavefunctions can be combined with a Kohn-Sham density functional theory (DFT)-based treatment of dynamic electron correlation. ${ }^{18-19}$ Many of these methods use conventional multiconfigurational wavefunctions to capture static correlation and include a DFT-based treatment of dynamic correlation. Methods of this type include rangeseparated wavefunction/DFT methods, ${ }^{20-21}$ multiconfigurational pair-density functional theory ${ }^{22-}$ 23 (MC-PDFT), and combinations of DFT and configuration interaction, ${ }^{24-25}$ such as in DFT/MRCI. ${ }^{26-27}$ Alternative approaches attempt to incorporate multireference character directly in the DFT formalism by using an unconventional reference ${ }^{28}$ or appealing to ensemble densities. Spin-flip TDDFT (SF-TDDFT) $)^{29}$ is an example of a scheme based on an unconventional reference. Spin contamination problems have plagued DFT-based spin-flip methods; ${ }^{30-31}$ however, this has recently been addressed by combining SF-TDDFT with ad hoc corrections from DFT/MRCI. ${ }^{32}$ Ensemble formulations of DFT include the spin-restricted ensemble-referenced Kohn-Sham 
(REKS) methods. ${ }^{33}$ To date, REKS methods need to be formulated specifically for the chosen ensemble (defined by an active space, as in CASSCF), and a general formulation applicable to arbitrary active spaces is lacking. Current implementations including gradients and nonadiabatic couplings are only applicable to an active space of two electrons in two orbitals. ${ }^{34-35}$

The recent development of particle-particle random phase approximation ( $p p$-RPA) methods has opened new possibilities for inexpensive excited-state methods. The original aim of $p p$-RPA was to provide ground state correlation energies via an adiabatic connection fluctuation dissipation theorem (ACFDT) approach. ${ }^{36-41}$ While the particle-hole RPA ( $p h$-RPA) ACFDT approach recovers the ring channel of the correlation energy from the coupled cluster doubles (CCD) equations, ${ }^{42}$ the ladder channel of the CCD correlation energy is obtained from the $p p$-RPA ACFDT approach. ${ }^{36,41}$ Weitao Yang and coworkers also highlighted $p p$-RPA and its TammDancoff approximated $p p$-TDA variant as effective methods to compute electronic ground and excited state energies. ${ }^{43-52}$ Starting from a doubly cationic $(N-2)$-electron reference, the $N$-electron ground state and excited states generated by excitations from the highest occupied molecular orbital (HOMO) are recovered by performing two-electron attachments. ${ }^{45,49-50}$ This allows the treatment of the $N$-electron ground and excited states on equal footing (derived as simultaneous eigenvalues of a common Hamiltonian) at a computational cost comparable to the simplest excited state methods, e.g., TDDFT/ph-RPA and configuration interaction singles (CIS). Because the ground and excited states are treated on equal footing, $p p$-RPA and $p p$-TDA based on an ( $N-2)$ electron reference are able to predict the correct topography around conical intersections, as has been shown explicitly for $\mathrm{H}_{3}$ and $\mathrm{NH}_{3} .{ }^{53}$ This is a major advance over conventional $p h$-TDDFT and CIS methods, which cannot reproduce conical intersections involving the ground state. ${ }^{2}$ In 2014, Peng et al. derived the $p p$-RPA equations from linear response theory by choosing a pairing field perturbation (termed TDDFT-P, see below in Section II.A). ${ }^{54}$ This formally allowed for the combination of $p p$-RPA and DFT references. Hence, the effect of dynamic correlation on the orbitals is incorporated through the exchange-correlation $(\mathrm{XC})$ potential, while the $p p$-RPA and $p p$-TDA schemes ensure that ground and excited states are treated on equal footing and therefore can treat exact degeneracies correctly (see also Section II.D).

In a procedure complementary to $p p$-TDA and $p p$-RPA based on an ( $N-2)$-electron reference, the $N$-electron ground state and excited states can also be generated through double annihilations from a double anionic $(N+2)$-electron reference in which the lowest unoccupied 
molecular orbital (LUMO) is populated with two additional electrons. This $(N+2)$-electron $p p$ RPA scheme and its corresponding hole-hole $(h h)$ Tamm-Dancoff approximation ( $h h$-TDA) were first presented by Yang and coworkers. ${ }^{49}$ Although the $p p$-RPA and $p p$-TDA methods based on an $(N-2)$-electron reference have now become quite established, ${ }^{43-53}$ less attention has been paid to the hh-TDA method based on an $(N+2)$-electron reference. Yang and coworkers applied the $h h$ TDA method to oxygen and sulfur atoms ${ }^{49}$ (noting "relatively large errors" in the results), but we have found no published reports of further developments or applications of $h h$-TDA to molecules. In this paper, we suggest that the $h h$-TDA method is worthy of renewed attention. Similar to the $p p$-TDA method, $h h$-TDA can effectively capture dynamic and static correlation in ground and low-lying excited states, including near- and exact degeneracies. Furthermore, the active orbital space in the $(N+2)$-electron-based $h h$-TDA appears to be suitable for the description of excited states in many organic molecules that are inaccessible to ( $N-2)$-electron $p p$-TDA. Examples include molecules with low-lying $n \pi^{*}$ and $\pi \pi^{*}$ excited states that cannot be simultaneously described within $p p$-TDA (which, by construction, can only describe excited states where an electron is excited from the HOMO).

In contrast to previous work on $p p-\mathrm{RPA}$, for both $p p$ - and $h h$-TDA, we formulate the response kernel in a functional-specific way that resembles the kernels occurring in linear density matrix response theory. This new formulation of the response kernel is compared to the previously used functional-independent variant. We put particular emphasis on the utility of the $h h$-TDA method in the treatment of organic and biologically relevant systems involving both $\pi \pi^{*}$ and $n \pi^{*}$ transitions.

\section{Theory}

\section{A. Particle-particle random-phase approximation from pairing field perturbations}

The particle-particle random phase approximation ( $p p$-RPA) equations were derived by Yang and coworkers ${ }^{54}$ by means of coupled time-dependent perturbation theory in analogy to wellestablished linear response theory by choosing a pairing field perturbation within the framework of Hartree-Fock/Kohn-Sham-Bogoliubov theory (termed TDDFT-P). ${ }^{55}$ The ground state for this non-interacting particle system is defined by the zero-temperature grand potential as: ${ }^{54-57}$

$$
\Omega[\gamma, \kappa]=T_{s}[\gamma, \kappa]+V_{\text {ext }}[\gamma]+D_{\text {ext }}[\kappa]+E_{\mathrm{JXC}}[\gamma, \kappa]-\mu N
$$


Here, $T_{s}[\gamma, \kappa]$ is the independent particle kinetic energy and $V_{\text {ext }}[\gamma]$ the external potential energy, which contains the nuclear-electron attraction. $D_{\text {ext }}[\kappa]$ denotes the external pairing potential and the last term preserves the total electron number. $E_{\mathrm{JXC}}[\gamma, \kappa]$ is the mean-field potential energy due to the electrons and includes the particle-hole and particle-particle channels via the one-particle density matrix $\gamma$ and the pairing matrix $\kappa$, respectively. The indices $\mathrm{J}, \mathrm{X}$, and $\mathrm{C}$ denote the Coulomb, exchange, and correlation components of this functional, respectively. The one-particle density matrix in the canonical molecular spin orbital basis is given by

$$
\gamma_{p q}=\left\langle\Psi\left|a_{p}^{\dagger} a_{q}\right| \Psi\right\rangle=\delta_{p q}, \forall p, q \in\{\uparrow, \downarrow\},
$$

while the pairing matrix (or anomalous density matrix) is defined as

$$
\kappa_{p q}=\left\langle\Psi\left|a_{p} a_{q}\right| \Psi\right\rangle, \kappa_{p q}^{*}=\left\langle\Psi\left|a_{p}^{\dagger} a_{q}^{\dagger}\right| \Psi\right\rangle, \forall p \in\{\uparrow\} \wedge q \in\{\downarrow\}
$$

$\Psi$ refers to the single-determinant ground state wavefunction of the non-interacting Kohn-Sham (KS) system. Here and in the following $i, j, k, l$ denote occupied, $a, b, c, d$ unoccupied, and $p, q, r, s$ general molecular orbitals.

For the hypothetical true functional $E_{\mathrm{JXC}}[\gamma, \kappa]$, the real-space density and anomalous density of the non-interacting KS system are identical to the respective quantities of the true interacting system. In the work of Peng et al., ${ }^{54}$ a non-superconducting system with no external pairing field in the ground state Hamiltonian is used. In that case, $D_{\text {ext }}[\kappa]=0$. As a result, the mean-field potential energy is free from indirect electron-electron interactions (e.g., phononmediated interactions) ${ }^{55}$ and contains only direct electron-electron interaction contributions via the (anti-symmetrized) Coulomb operator and XC potential. Based on the ground state defined by Eq. 1 , Peng et al. derived the $p p$-RPA method by perturbing the ground state with an external pairing field perturbation. The TDDFT-P equations, which describe the coupled response to the pairing field perturbation, are given as: ${ }^{54}$

$$
\begin{aligned}
& -\left[\omega-\left(\epsilon_{i}+\epsilon_{k}-2 \mu\right)\right] \delta \kappa_{i k}(\omega)=\delta D_{i k}^{\mathrm{ext}}(\omega)-\delta D_{i k}^{\mathrm{JXC}}(\omega) \\
& {\left[\omega-\left(\epsilon_{a}+\epsilon_{c}-2 \mu\right)\right] \delta \kappa_{a c}(\omega)=\delta D_{a c}^{\mathrm{ext}}(\omega)-\delta D_{a c}^{\mathrm{JXC}}(\omega)}
\end{aligned}
$$

Here, $\delta \kappa_{p q}(\omega)$ are the first order changes in the pairing matrix induced by the external frequencydependent pairing field perturbation $\delta D_{p q}^{\mathrm{ext}}(\omega), \omega$ is the frequency of the perturbation, $\epsilon_{p}$ the 
orbital energy, and $\mu$ is the chemical potential or Fermi energy. The pairing matrix response $L_{p q, r s}$ of the mean field potential is contained in $\delta D_{p q}^{\mathrm{JXC}}(\omega)$.

$$
\delta D_{p q}^{\mathrm{JXC}}(\omega)=\sum_{j>l}^{N_{\text {occ }}} L_{p q, j l} \delta \kappa_{j l}(\omega)+\sum_{b>d}^{N_{\text {vit }}} L_{p q, b d} \delta \kappa_{b d}(\omega)
$$

In our formulation of $p p$-RPA, the choice of $L_{p q, r s}$ differs from the one presented by Yang and coworkers, ${ }^{44-45,54}$ as discussed in detail below.

\section{B. The adiabatic particle-particle linear-response kernel}

For a non-superconducting system in the absence of $D_{\text {ext }}[\kappa]$, the pairing matrix and its associated $\mathrm{XC}$ potential are zero in the electronic ground state. Consequently, only the $\gamma$-dependent terms in Eq. 1 survive and minimizing the zero-temperature grand potential becomes equivalent to minimizing the standard KS or Hartree-Fock (HF) energy expression. It is only after taking the second derivatives with respect to $\kappa$ that the particle-particle channels of the mean-field potential as in Eqs. 4 and 5 become non-zero. In the framework of ab initio wavefunction theory, the $p p$ RPA equations are based on a HF reference and the mean-field potential takes the form of an antisymmetrized Coulomb integral. ${ }^{41}$ In that case, the $p p$ and particle-hole $(p h)$ channels of the meanfield potential are formally equivalent. We note, however, that only the exchange-type integral survives the spin integration in the $p p$ channel (see supporting information, $\mathrm{SI}$ ).

In the context of approximate KS DFT, there may exist some liberty in choosing the explicit form of this response kernel. Given that contemporary semi-local density functional approximations are defined for $\kappa=0$, the pairing matrix response of the density functional drops out:

$$
E_{\mathrm{XC}}^{\mathrm{DFA}}[\gamma, \kappa=0]=E_{\mathrm{XC}}^{\mathrm{DFA}}[\gamma] \rightarrow \frac{\partial^{2} E_{\mathrm{XC}}[\gamma]}{\partial \kappa_{p q}^{*} \partial \kappa_{r s}}=0
$$

Yang and coworkers have already employed this approximation for the XC density functional response. Furthermore, they chose to use the HF-type mean-field response, i.e., a bare antisymmetrized Coulomb integral, as the response kernel. ${ }^{54}$ It is therefore independent of the underlying density functional approximation (DFA). With this kernel, the chosen DFA only affects the orbitals and orbital energies that enter the $p p$-RPA calculation, but not the $p p$ response expression itself. 
In this work, we employ a different choice for the $p p$ response kernel. Given the formally equivalent $p h$ and $p p$ response kernels when using a HF reference, ${ }^{41}$ which may be regarded as a special choice of the KS system, we draw analogy from the well-known ph linear response TDDFT. In spin-preserving ${ }^{1,58}$ and spin-flip (SF) ${ }^{29}$ formulations of TDDFT, the response kernel reflects the underlying ground state DFA in the way the non-local Fock exchange enters the response. Hence, we choose to use the same modification of the non-local Fock exchange, i.e., the global scaling and/or range-separation employed by the corresponding DFA.

$$
L_{p q, r s}=[p s \mid q r]-a_{X}^{\mathrm{FR}}[p r \mid q s]-a_{X}^{\mathrm{LR}}[p r \mid q s]^{\mathrm{LR}}
$$

The first term on the right-hand side corresponds to the Coulomb-type two-electron integral (Mulliken notation for spin MOs), whereas the remaining terms denote the modified exchangetype two-electron integrals. $a_{X}^{\mathrm{FR}}$ is the functional-specific scaling factor for the global (full-range, FR) exchange integral

$$
[p r \mid q s]=\iint \phi_{p}^{*}\left(\mathbf{x}_{1}\right) \phi_{r}\left(\mathbf{x}_{1}\right) \frac{1}{r_{12}} \phi_{q}^{*}\left(\mathbf{x}_{2}\right) \phi_{s}\left(\mathbf{x}_{2}\right) \mathrm{d} \mathbf{x}_{1} \mathrm{~d} \mathbf{x}_{2} .
$$

Here, $\phi$ refers to a molecular spin orbital and $\mathbf{x}_{1} / \mathbf{x}_{2}$ denote all spatial and spin coordinates of electrons $1 / 2$. The long-range (LR) part of the exchange integral employs the modified Coulomb operator and is given as

$$
[p r \mid q s]^{\mathrm{LR}}=\iint \phi_{p}^{*}\left(\mathbf{x}_{1}\right) \phi_{r}\left(\mathbf{x}_{1}\right) \frac{\operatorname{erf}\left(\omega_{\mathrm{LR}} r_{12}\right)}{r_{12}} \phi_{q}^{*}\left(\mathbf{x}_{2}\right) \phi_{s}\left(\mathbf{x}_{2}\right) \mathrm{d} \mathbf{x}_{1} \mathrm{~d} \mathbf{x}_{2} .
$$

Like Yang and coworkers, we also assume the pairing matrix response of the $\mathrm{XC}$ functional to be zero (see Eq. 7), allowing the response kernel to become frequency independent. Due to spin integration (note the definition of the pairing matrix), the Coulomb part in the $p p$ response kernel is zero, hence

$$
L_{p \bar{r}, r \bar{s}}=-a_{X}^{\mathrm{FR}}(p r \mid \overline{q s})-a_{X}^{\mathrm{LR}}(p r \mid \overline{q S})^{\mathrm{LR}} .
$$

Here, we have used the Mulliken notation for spatial MOs and overbarred orbital indices to highlight orbitals corresponding to $\beta$ spin. Our choice for the response kernel (Eq. 11) is identical to the one in collinear SF-TDDFT but with inverted sign (note that $L_{p q, r s}$ is subtracted in Eq.14 below). This can be understood from a second quantization picture in which both the spin-flip and $p p$-RPA approaches apply creation/annihilation operators acting on different spin, i.e., $a_{\uparrow}^{\dagger} a_{\downarrow}$ in 
spin-flip and $a_{\uparrow}^{\dagger} a_{\downarrow}^{\dagger} / a_{\uparrow} a_{\downarrow}$ in the $p p / h h$ channels. Therefore, only the exchange integral does not vanish upon spin integration, as in $p p$-RPA, and the flipped sign results from the commutation relation of the annihilation and creation operators (see SI for a comparison). SF-TDDFT can be derived from linear-response $p h$ TD-DFT, if changes in the density matrix that do not preserve the $\hat{S}_{z}$ expectation value are allowed. ${ }^{29}$ Due to its origin from an analogy to linear response SFTDDFT, we refer to our choice of the response kernel, Eq. 11, as the linear response-type kernel in the following to distinguish it from the DFA-independent, HF-like kernel used by Yang and

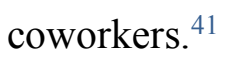

\section{The hole-hole Tamm-Dancoff approximated pp-RPA method}

Using the response kernel in Eq.11, we can rewrite Eqs.4 and 5 in matrix notation as

$$
\left[\left(\begin{array}{cc}
\mathbf{A}^{p p} & \mathbf{B}^{p h} \\
\mathbf{B}^{h p} & \mathbf{A}^{h h}
\end{array}\right)-\left(\begin{array}{cc}
\omega & 0 \\
0 & -\omega
\end{array}\right)\right]\left(\begin{array}{c}
\boldsymbol{\kappa}^{p p}(\omega) \\
\boldsymbol{\kappa}^{h h}(\omega)
\end{array}\right)=\left(\begin{array}{c}
\delta \mathbf{D}^{p p}(\omega) \\
\delta \mathbf{D}^{h h}(\omega)
\end{array}\right) .
$$

Here, the superscripts $p p$ and $h h$ define the particle-particle (double creation in the virtual space) and hole-hole (double annihilation in the occupied space) blocks, respectively. $\mathbf{A}^{p p}$ is of dimension $n_{\mathrm{virt}}^{2} \times n_{\mathrm{virt}}^{2}$ and $\mathbf{A}^{h h}$ is of dimension $n_{\mathrm{occ}}^{2} \times n_{\mathrm{occ}}^{2}$. Their elements are given as

$$
\left[\mathbf{A}^{p p}\right]_{a c, b d}=A_{a c, b d}=\delta_{a b} \delta_{c d}\left(\epsilon_{a}+\epsilon_{c}-2 \mu\right)-L_{a c, b d}
$$

and

$$
\left[\mathbf{A}^{h h}\right]_{i k, j l}=A_{i k, j l}=-\delta_{i j} \delta_{k l}\left(\epsilon_{i}+\epsilon_{k}-2 \mu\right)-L_{i k, j l} .
$$

$\mathbf{B}^{h p}=\left[\mathbf{B}^{p h}\right]^{T}$ is of dimension $n_{\mathrm{occ}}^{2} \times n_{\mathrm{vit}}^{2}$ and is given as

$$
B_{i k, b d}=-L_{i k, b d} \text {. }
$$

If we now let the perturbation (right-hand side in Eq. 12) go to zero, we find that there are nontrivial solutions to this system of equations, which correspond to the poles of the particleparticle/hole-hole response function. This leads to the non-Hermitian eigenvalue problem

$$
\left(\begin{array}{ll}
\mathbf{A}^{p p} & \mathbf{B}^{p h} \\
\mathbf{B}^{h p} & \mathbf{A}^{h h}
\end{array}\right)\left(\begin{array}{l}
\mathbf{X} \\
\mathbf{Y}
\end{array}\right)=\left(\begin{array}{c}
\mathbf{X} \\
\mathbf{Y}
\end{array}\right)\left(\begin{array}{cc}
\boldsymbol{\omega} & 0 \\
0 & -\boldsymbol{\omega}
\end{array}\right) .
$$

Employing the commonly used notation, ${ }^{54}$ the matrices $\mathbf{X}$ and $\mathbf{Y}$ contain the eigenvectors, which correspond to the $p p$ and $h h$ changes in the pairing matrix $\kappa . \omega$ is a diagonal matrix, which contains the eigenvalues of the non-Hermitian eigenvalue problem. If we neglect the coupling matrices $\mathbf{B}^{h p}$ 
and $\mathbf{B}^{p h}$ between the $p p$ and $h h$ blocks, we obtain the so-called Tamm-Dancoff approximated (TDA) eigenvalue problems:

$$
\mathbf{A}^{p p} \mathbf{X}=\mathbf{X} \omega^{p p}
$$

for the $p p$-TDA case and

$$
\mathbf{A}^{h h} \mathbf{Y}=\mathbf{Y} \boldsymbol{\omega}^{h h}
$$

for the $h h$-TDA problem.

Note that within TDA, the constant diagonal shift of $\mp 2 \mu$ in Eqs. 13 and 14 can be removed from the definition of the corresponding matrix elements. Both of these eigenvalue problems are guaranteed to have only real eigenvalues. This makes them more robust than the full $p p$-RPA case, which can have complex solutions that spoil the potential energy surfaces around conical intersections (as noted in the context of coupled cluster theory ${ }^{59-60}$ ). While the $p p$-TDA method based on an $(N-2)$-electron reference has been extensively investigated as a method to describe low-lying excited states ${ }^{45,49}$ and $\mathrm{S}_{0} / \mathrm{S}_{1}$ conical intersections, ${ }^{53}$ the $h h$-TDA method has been less thoroughly explored for molecular electronic structure. ${ }^{49}$ In this work, we suggest $h h$-TDA should be revisited as an efficient DFT-based method capable of computing low-lying excited states even in the presence of conical intersections.

Before benchmarking the $h h$-TDA method along with our choice for the response kernel (Eq.11), we will briefly discuss some practical aspects of the $p p$-TDA and $h h$-TDA methods in the next section.

\section{Practical considerations of the pp-TDA and hh-TDA methods}

In both the $p p$-TDA and $h h$-TDA methods, the $N$-electron target system is described by first solving the ground state electronic structure for a system that differs by two electrons from the $N$-electron target system. This corresponds to a double cation ( $N-2$ electrons) in the case of $p p$-TDA or a double anion $(N+2$ electrons) in the case of $h h$-TDA. The ground and excited states of the $N$-electron target system are then obtained by creation ( $p p$-TDA) or annihilation ( $h h$-TDA) of two electrons (see Figure 1).

The inability to describe either doubly excited states or conical intersections involving the ground state are well-known shortcomings of standard single-reference methods like linearresponse $(p h)$ TD-DFT and CIS. These are both rectified by $p p$ - and $h h$-TDA. Since the ground and the excited states are solutions of the same eigenvalue problem, near- and exact degeneracies 
(such as conical intersections) can be properly described. Furthermore, some doubly excited states can be computed (see Figure 2). At the same time, the preceding ground state calculation of the double ionic state is of single-reference complexity and incorporates dynamic correlation by virtue of the XC potential. Therefore, both $p p$ - and $h h$-TDA schemes are in principle able to capture static and dynamic correlation, making them promising methods for use in nonadiabatic dynamics simulations. Furthermore, in contrast to the SF-TDDFT scheme, pure eigenfunctions of $\hat{S}^{2}$ are obtained trivially in both $p p$ - and $h h$-TDA.

However, both methods also have some obvious shortcomings in common. First, the excited state expansion space is highly restricted. Taking the $N$-electron target system as reference, $p p$-TDA only includes excitations from the HOMO, while $h h$-TDA is restricted to excitations to the LUMO. Second, both methods take a detour by computing the orbitals for a reference that differs in its total charge from the target state, as SF-TDDFT does with a differing spin state. As a result, two-state degeneracies of the $N$-electron state can be treated at the cost of a single reference method. However, the orbitals are not optimized for the $N$-electron system. This may have a significant implication for dynamics simulations: a degeneracy on the $(N+2)$ or $(N-2)$ surface itself can occur and cause instabilities in the SCF procedure.

Zhang et al. noted that the choice of a $(N+2)$ reference may be hampered by the existence of unbound orbitals. ${ }^{50}$ Presumably, this is one of the reasons that the $h h$-TDA method has not received serious attention in molecular electronic structure so far. There are two distinct consequences of unbound orbitals that can be envisioned. The first consequence is quite practical, namely that it may be difficult to converge the SCF procedure for the $(N+2)$ reference. In the usual $h h$-TDA method, non-convergence of the SCF procedure for the $(N+2)$ reference would be fatal. The second consequence is somewhat more formal. Even if SCF convergence can be achieved, orbitals with positive orbital energies do not correspond to bound electrons. They can thus be very sensitive to the employed basis set. Indeed, with a sufficiently flexible basis set, they would be expected to be highly delocalized continuum functions and poor representatives of a low-lying valence excited state.

Regarding the first consideration, we note that poor convergence of the SCF procedure will be exacerbated if the long-range potential is incorrect. DFAs with 100\% long-range Fock exchange are known to capture the asymptotic potential correctly. Accordingly, using DFAs with full longrange exchange, we have not observed any instability of the $(N+2)$-electron SCF procedure for the 
molecules we tested. Even the green fluorescent protein chromophore (HBI) anion with a net charge of -3 for the reference state converges without difficulty when an asymptotically correct, range-separated DFA is used. However, the $h h$-TDA method is likely to encounter serious SCF convergence difficulties for DFAs that do not incorporate long-range exact exchange.

It has been previously observed that finite-basis set DFT is capable of providing reasonable electron affinities computed as energy differences $\mathrm{EA}=E_{\mathrm{N}}-E_{\mathrm{N}+1}$, even when the anion has unbound occupied orbitals. ${ }^{61}$ This suggests that the second consideration is largely formal. However, there are also some practical concerns. The success of $h h$-TDA in describing excited states is predicated on the HOMO orbital of the $(N+2)$-electron determinant being a good approximation to the bound orbital that predominantly contributes to the low-lying $N$-electron excited states. This will certainly not hold if the HOMO is a good approximation to a continuum orbital, which is the expected outcome in a sufficiently large basis set. We take a practical approach here and recommend that diffuse basis sets should be avoided in the context of $h h$-TDA. For the finite orbital basis sets of double- or triple-zeta quality used in this study, there are very few cases where the $h h$-TDA scheme based on an $(N+2)$-electron reference fails, in spite of the presence of occupied orbitals with positive energies. This implies that the HOMO of the $(N+2)$-electron system is a good approximation to the LUMO of the N-electron system, albeit with shifted orbital energies. This shift is effectively removed during the $h h$-TDA step, leading to a reasonable description of the relative energies between the $N$-electron ground and low-lying excited states. We will further elaborate on this issue in Section IV.E, but we emphasize again that diffuse basis sets should usually be avoided in combination with the $(N+2)$-electron reference calculation.

For $p p$-TDA, there may also exist a practical implication with respect to the underlying DFA. It was shown that $p p$-RPA based on a HF reference leads to less accurate singlet-triplet splittings, ${ }^{48}$ slower basis set convergence, ${ }^{45}$ and overall less accurate excitation energies for small molecules $^{45}$ than $p p$-RPA based on DFAs with little or no exact exchange. Similar results for excitation energies were reported for $p p$-TDA. ${ }^{45}$ A convenient test case is the $\pi \pi^{*}$ state of ethylene, where both $h h$-TDA and $p p$-TDA provide a suitable active orbital space. Accurate methods such as coupled cluster and multireference perturbation theory agree that this should be the lowest valence excited state. For ethylene, we observe a stronger dependence on the amount of Fock exchange in the DFA for $p p$-TDA (Table S13 in the SI). The $\pi \pi^{*}$ state is the fourth excited state for pp-TDA-HF, while it is the second excited state for hh-TDA-HF. It is well-known that adding 
Fock exchange to a DFA increases the differential treatment of virtual and occupied orbitals. In molecular systems, ${ }^{62}$ the virtual orbitals in HF are best suited to describe electron attachment, while virtual orbitals in (semi-)local DFT are more appropriate to describe electronic excitations. ${ }^{63}$ This implies that DFAs with large amounts of exact exchange will raise the energy of the HOMO of the $\mathrm{N}$-electron target system in $\mathrm{pp}$-TDA. Therefore, when using practical DFAs, the optimal amount of exact exchange could be different for $p p$-TDA and $h h$-TDA and this point deserves further study. In this work, we focus on DFAs for the linear-response-type kernel $h h$-TDA framework.

We will note (details in Table S13) that the ordering of the $\pi \pi^{*}$ state for ethylene in $p p$ TDA is improved by using our linear response-type kernel compared to the previously introduced functional-independent HF-type response kernel. ${ }^{45,49,54}$ For clarity, it should be noted that purely (semi-)local DFAs have a vanishing response kernel in our linear-response-type formulation, i.e., $\mathbf{A}^{p p}$ and $\mathbf{A}^{h h}$ are diagonal in that case. In the HF-type response kernel, the bare anti-symmetrized Coulomb integral is used as the response kernel, regardless of the underlying DFA.

In $h h$-TDA, within the limitations of the given basis set (see above), the LUMO (with respect to the $N$-electron target system) is generated on equal footing with the occupied orbitals for HF or any DFA. Another difference between $h h$ - and $p p$-TDA is the dimension of the A matrix to be diagonalized. In $p p$-TDA, this dimension increases strongly with the size of the atomic orbital (AO) basis. For $h h$-TDA, the dimensions are independent of the basis set size (unless effective core potentials are used).

We mention for the sake of completeness, that $p p$-TDA-HF is equivalent to full configuration interaction (FCI) for any two-electron system, while $h h$-TDA-HF is equivalent to FCI for an $N$-electron system in a basis that provides exactly two virtual molecular spin orbitals. We have confirmed this via calculations on $\mathrm{H}_{2}$ in an STO-3G basis set (see SI). Thus, both schemes have similarities to CASCI methods with a fixed active space (i.e., $N$ electrons in $N / 2+1$ orbitals for $h h$-TDA and two electrons in $n_{\text {virt }}+1$ orbitals for $p p$-TDA). One can speculate that the ability of the $p p$-TDA and $h h$-TDA to describe static correlation arises from their similarity to CASCI methods. For the same reason, however, neither $p p$-TDA nor $h h$-TDA are size consistent. As long as no degeneracy of the LUMO/LUMO+1 (hh-TDA) or the HOMO/HOMO-1 ( $p p$-TDA) is present, this is not expected to have practical implications for the description of relative energies between electronic states. 


\section{Computational Details}

The $h h$-TDA and $p p$-TDA methods have been implemented in the electronic structure code TeraChem. ${ }^{64-65}$ All $h h$-TDA and $p p$-TDA calculations in this work are performed with this development version of TeraChem. To compare with the $p p$-TDA data in the literature, we have implemented our choice for the response kernel (Eq.11) as well as the HF-type response kernel of Yang and coworkers. ${ }^{54}$ We confirmed that our implementation is correct by comparison of $p p$ TDA excitation energies with values reported in the literature ${ }^{45}$ as well as comparisons between CASCI and $h h / p p$-TDA using Hartree-Fock reference states.

In the functional assessment for vertical excitation energies with $h h$-TDA in Section IV.A, we considered the global hybrid functionals B3LYP, ${ }^{66-69}$ PBE0, ${ }^{70-71}$ and BHLYP ${ }^{66-67,72}$ as well as Hartree-Fock. Thus, we cover different amounts of non-local Fock exchange in the mean field Hamiltonian $(20 \%, 25 \%, 50 \%$, and $100 \%$, respectively). Additionally, we test different rangeseparated hybrid functionals, namely CAM-B3LYP, ${ }^{66-69,}{ }^{73} \omega \mathrm{PBEh},{ }^{74}$ and the $\mathrm{B} 97^{75}$ type functionals: $\omega \mathrm{B} 97,{ }^{76} \omega \mathrm{B} 97 \mathrm{X},{ }^{76}$ and $\omega \mathrm{B} 97 \mathrm{X}-\mathrm{D} 3 .{ }^{77}$ For clarity, it is emphasized that the latter two are different in their functional parameters (see SI), and hence, should give rise to different electronic structures. The DFA assessment is restricted to the linear response-type kernel variant of $h h$-TDA (Eq.11) For the assessment of different functionals, we employed the spherical splitvalence atomic orbital def2-SV(P) ${ }^{78-79}$ basis set by Ahlrichs and coworkers. All SCF calculations used the converged Hartree-Fock orbitals as guess orbitals. The systems considered in this work are given in the SI.

When comparing $p p$-TDA and $h h$-TDA in Section IV.B, we use the same spherical TZVP basis set ${ }^{80}$ that has been used in the original benchmark papers by Thiel and coworkers. ${ }^{81}$ Both schemes are employed in a setting, in which the occupied and active orbitals have been generated on equal footing, i.e., where these orbitals experience a mean-field potential with the same number of electrons. The $p p$-TDA is hence combined with the generalized gradient approximation (GGA) functional $\mathrm{PBE}^{70}$ (see also Section II.D). $h h$-TDA is combined with the range-separated functional $\omega \mathrm{B} 97 \mathrm{X},{ }^{76}$ which is shown to be a well-performing combination in Section IV.A (and in the SI). We consider both the linear response-type (this work) and the HF-type (Yang and coworkers) response kernels for $p p$-TDA and $h h$-TDA. Additionally, we have performed $p h$ Tamm-Dancoff approximated TDDFT calculations with the $\omega$ B97X functional. For reference, we use the best estimates for excitation energies of Thiel and coworkers ${ }^{81}$ and complement these with equation- 
of-motion singles and doubles coupled cluster (EOM-CCSD) calculations (same basis set) as implemented in Q-Chem v5.0.0. ${ }^{82}$

In Section IV.C.1, we calculate the ethylene potential energy surface (PES) with $h h$-TDA$\omega B 97 X$ (linear response kernel) using the Cartesian def2-SVP ${ }^{78-79}$ basis set. The scans along the pyramidalization and torsion angles were performed without relaxation of the remaining degrees of freedom.

The excited state energies of thymine at three critical structures are considered in Section IV.C.2. We use $h h$-TDA in combination with the $\omega$ PBE functional and the Cartesian 6-31G** basis set. ${ }^{83-85}$ A range separation parameter $\omega=0.2$ a.u. was selected after a coarse scan and comparison against high level reference values for this molecule. We calculated these reference values with EOM-CCSD/aug-cc-pVDZ with Q-Chem. While the Franck-Condon (FC), the $\mathrm{S}_{1}$ minimum, and the $\mathrm{S}_{1} / \mathrm{S}_{2}$ minimum energy conical intersection (MECI) were optimized with $h h$ TDA, we used the respective coupled cluster (CC) geometries of Ref. 86 for single-points at the EOM-CCSD/aug-cc-pVDZ level of theory. The $\mathrm{S}_{2}\left(\pi \pi^{*}\right)$ saddle point geometry therein is claimed to be "in close proximity" to the conical intersection seam. ${ }^{86}$ Due to the lack of a properly CCoptimized MECI structure, we use that structure as a substitute and estimate the MECI energy by averaging the $\mathrm{S}_{1}$ and $\mathrm{S}_{2}$ energies computed with EOM-CCSD/aug-cc-pVDZ.

In Section IV.C.3, we apply $h h$-TDA with the $\omega \mathrm{B} 97^{76}$ functional in combination with the Cartesian $6-31 \mathrm{G}^{* *}$ basis set ${ }^{83-85}$ and compare to results obtained with multireference configuration interaction methods including single and double excitations (MRSDCI). ${ }^{87}$

\section{Results}

\section{A. Functional benchmarking for hh-TDA}

The performance of density functionals for the calculation of vertical excitation energies in the linear-response $p h$ time-dependent density functional theory framework is well known ${ }^{88}$ and the role of Fock exchange is fairly well understood. ${ }^{1}$ In contrast, for the $h h$-TDA method proposed in this work, the impact of Fock exchange and the use of an anionic reference on the excitation energies is not known. Therefore, we start by benchmarking several common DFAs along with Hartree-Fock (HF) in the calculation of vertical excitation energies with $h h$-TDA. 
To gain more insight, we consider the lowest vertical excitation energies using molecules and reference data from previously published data sets where highly accurate excitation energies are available. ${ }^{81,89-90}$ We classify the excitation type into different categories: intermolecular charge-transfer (CT) and predominantly local excitations. We also consider molecules that have push-pull type excitations, i.e., excitations with partial intramolecular CT character. We find that the functionals behave similarly for this set as for the local excitations. Thus, we have added them to the latter set and distinguish only intermolecular CT and intramolecular excitations (see SI for separate results of the local and the push-pull set). In the latter set, we have selected molecules for which $h h$-TDA, across different DFAs, produces the same character for the lowest vertical excitation as the reference method (SCS-CC2 ${ }^{91}$ ).

We have not considered any purely semi-local GGA-type DFAs, since these show severe convergence problems in the self-consistent field (SCF) procedure of the double anionic reference (see also Section II.D). Though we also expect that global hybrids will not be optimal in that case, for completeness we have included some prototypical global hybrid functionals that differ in the amount of Fock exchange. All functionals considered are listed in Section III, but we restrict the discussion in this paper to the globally constant Fock-exchange DFAs B3LYP, BHLYP, and HF (these functionals contain $20 \%, 50 \%$, and $100 \%$ of Fock exchange, respectively). Furthermore, we find that all tested range-separated DFAs with $100 \%$ long-range exchange perform similarly; therefore, we restrict the current discussion to the $\omega \mathrm{B} 97 \mathrm{X}-\mathrm{D} 3$ functional. The results for the other functionals that were tested can be found in the SI.

Figure 3 shows the Gaussian error distributions based on the mean deviation (MD) and standard deviation (SD) obtained for the aforementioned reference data sets of excitation energies. While $h h$-TDA-HF yields CT excitation energies that are on average underestimated by almost 1 $\mathrm{eV}$, the excitation energies for locally excited states show a systematic overestimation of about the same magnitude. Furthermore, the error is significantly more systematic for the intermolecular CT excitations. For DFAs with low amounts of Fock exchange, i.e., B3LYP, these trends are reversed, however, with a smaller magnitude for the underestimation of local excitation energies. It is noteworthy that the DFAs behave quite different than in a $p h$ linear response TD-DFT framework. In the latter, DFAs with low amounts of Fock exchange significantly underestimate CT excitations. ${ }^{89}$ The BHLYP functional, which is in between in terms of the percentage of Fock exchange, shows more consistent errors for both sets $(\mathrm{MD} \approx 0.4-0.5 \mathrm{eV})$, as well as more narrow 
error distributions. The range-separated DFA $\omega B$ B7X-D3 behaves even slightly better than BHLYP. This, along with the fact that the $100 \%$ asymptotic Fock exchange makes these DFAs less prone to SCF convergence issues with the $(N+2)$-electron reference, suggests the use of asymptotically correct range-separated hybrid functionals in combination with the $h h$-TDA scheme.

In addition to benchmarking the DFAs for CT and local states, we investigate state splittings between $\pi \pi^{*}$ and locally excited states with different character (mostly $n \pi^{*}$ ). For this purpose, we have selected a set of systems mostly comprised of molecules from Thiel's 2008 benchmark set. ${ }^{81,90}$ Problematic systems, which are impossible to treat with $h h$-TDA due to the restricted orbital space, were discarded from the benchmark set. These are systems in which either the population of the frontier orbitals in the $(N+2)$-electron system is not clear (degenerate LUMOs in the $N$-electron determinant) or the excited states cannot predominantly be described by a single orbital-orbital transition (benzene, naphthalene, pyrrole, s-triazine). Furthermore, butadiene, hexatriene, and octatetraene are not considered here but in Section IV.B. The results are visualized in Figure 4, while the respective symmetry labels and results for other density functionals can be found in the SI. First, we stress the relevance of high amounts of Fock exchange in the DFA, which we mentioned earlier in Section II.D. We find that B3LYP and BHLYP, but also a few rangeseparated functionals (CAM-B3LYP or $\omega \mathrm{PBEh}$, see SI), show incorrect orbital occupations for some carbonyl systems in the $(N+2)$-electron SCF calculation, i.e., the HOMO of the $(N+2)$ electron system does not correspond to the LUMO obtained from an SCF calculation on the $N$ electron system. Hence, the excited states for these systems cannot be described properly with these functionals.

We also find that the global hybrid functionals perform poorly for the state splittings compared to Hartree-Fock or range-separated functionals (e.g., wB97X-D3). Except in two cases ( $\mathrm{n} \pi^{*} / \pi \pi^{*}$ in aspirin and $\sigma \pi^{*} / \pi \pi^{*}$ in cyclopropane), the latter two perform similarly. Both describe the $n \pi^{*}$ splittings reasonably well, while systematically overestimated $\pi \pi^{*}$ excitations are found for aromatic systems, regardless of which DFA is used. This seems to be caused by the absence of accessible higher lying anti-bonding $\pi$ orbitals in the expansion space, which would lower these excitations and improve the energetic splittings with respect to the $n \pi^{*}$ excitations. This is also observed for the splittings between the different $\pi \pi^{*}$ states in cyclopentadiene and norbornadiene, presumably for the same reasons. Interestingly, reduced short-range Fock exchange leads to 
increased $\sigma \pi^{*}$ excitation energies in cyclopropene, which then lead to qualitatively incorrect energy splittings with respect to the $\pi \pi^{*}$ state. This is observed for all DFAs and only HF ranks these states qualitatively correctly.

Overall the results indicate that the $h h$-TDA scheme in combination with a range-separated hybrid functional appears to be a promising method for describing the ground and low-lying excited states of organic molecules on equal footing.

B. Comparison of pp-TDA and hh-TDA with linear response-type and full exchange response kernels

In this section, we compare the $h h$-TDA and $p p$-TDA schemes as well as the two different response kernel choices. In Table 1, we have tabulated the statistical data for excitation energies computed for a subset of excitations from the Thiel benchmark set ${ }^{81}$ with the linear response (LR) and HF-type kernels for $h h$-TDA- $\omega \mathrm{B} 97 \mathrm{X}$ and $p p$-TDA-PBE. We note that our $p p$-TDA-PBE (HF) results are in agreement with the ones presented earlier by Yang and coworkers (note that we use a slightly different basis set here). ${ }^{45}$ The statistical data is listed in Table 1, while detailed values of the individual systems and states are given in the SI. A significant difference between the $h h$ TDA and $p p$-TDA schemes becomes apparent when looking at systems that involve both $\pi \pi^{*}$ and other types of excitations (i.e., $n \pi^{*}$ or $\sigma \pi^{*}$ ). Here, we find that $h h$-TDA typically provides the better option, since the configuration space in $h h$-TDA is more suited to describe both excitation types simultaneously. This manifests itself in a larger number of states on this set, which can actually be described by $h h$-TDA. The nucleobases and carbonyl systems are particularly good cases for $h h$-TDA (see SI). For $p p$-TDA-PBE, SCF convergence problems for these systems eliminate the possibility to include them in the analysis. This seems to be due to (nearly) degenerate frontier molecular orbitals in the $(\mathrm{N}-2)$ reference and was confirmed using alternative electronic structure software (Turbomole version 6.4) ${ }^{74,92}$ The HF-type variant performs better for $p p$-TDAPBE than the LR variant, the latter being equivalent to plain PBE orbital energy differences. For $h h$-TDA- $\omega \mathrm{B} 97 \mathrm{X}$, this is reversed and the LR-type kernel performs better, as reflected by smaller mean (absolute) and standard deviations. Both $p p$-TDA-PBE schemes on average show underestimated excitation energies, whereas the opposite is true for $h h$-TDA- $\omega \mathrm{B} 97 \mathrm{X}$. From all of the considered $p p / h h$ schemes, $h h$-TDA- $\omega \mathrm{B} 97 \mathrm{X}$ (LR) performs the best on this set. 
Compared to regular $p h$-TDA- $\omega \mathrm{B} 97 \mathrm{X}$ and EOM-CCSD, $h h$-TDA- $\omega \mathrm{B} 97 \mathrm{X}$ (LR) provides almost comparable accuracy, with a slightly less systematic error distribution as reflected by a SD, which is twice as large as for $p h$-TDA- $\omega \mathrm{B} 97 \mathrm{X}$. In summary, we find that $h h$-TDA provides a reasonable method typically with a broader applicability to many organic systems compared to $p p$ TDA. In the next sections, we will only consider the LR-type kernel variant of $h h$-TDA and investigate potential energy surface properties beyond the Franck-Condon point.

\section{Potential energy surfaces with hh-TDA}

1. Ethylene

As addressed in the previous sections, one of the main advantages of the $h h$-TDA method is the ability to accurately treat degeneracies involving the ground state and low-lying excited states. The topologically correct description of the potential energy surfaces in regions of degeneracy is especially important in nonadiabatic photochemical dynamics where population transfer between adiabatic potential surfaces is often mediated by these seams of conical intersection. A prototypical example of such an intersection can be accessed through torsion and pyramidalization degrees of freedom in ethylene; this particular case has previously been used as a model system for assessing the ability of electronic structure methods to describe statically correlated character and ground-excited state degeneracies. ${ }^{2}$

Typical low-cost single reference excited state methods, such as CIS and TDDFT, suffer from instability related to HOMO-LUMO orbital degeneracies as an MECI involving the ground state and an excited state is approached. As a result, these methods cannot properly describe the twisted-pyramidalized MECI in ethylene. By contrast, $h h$-TDA is well-suited to describing conical intersection topologies, as demonstrated in combination with the $\omega \mathrm{B} 97 \mathrm{X}$ functional in Figure 5a, in which the double cone topology characterizes the vicinity of the $\mathrm{S}_{0} / \mathrm{S}_{1}$ MECI. Here, the potential energy surfaces are plotted along torsion and pyramidalization coordinates, which are the branching plane coordinates corresponding to the twisted-pyramidalized minimum energy conical intersection (MECI) between the $\mathrm{S}_{0}$ ground state and the $\pi \pi^{*}$ excited state.

Moreover, CIS and TDDFT cannot describe double excitations, which then leads to a description of the $\mathrm{S}_{1}$ minimum at a purely twisted geometry $\left(90^{\circ}\right.$ torsion, no pyramidalization). ${ }^{2}$ Methods that incorporate doubly excited states describe the $\mathrm{S}_{1}$ minimum as simultaneously twisted $\left(90^{\circ}\right.$ torsion) and pyramidalized. $h h$-TDA captures this feature of the $\mathrm{S}_{1}$ potential energy surface (PES), as demonstrated by the appearance of the minimum at a twisted $\left(90^{\circ}\right)$ and pyramidalized 
$\left(60^{\circ}\right)$ geometry that lies $4.76 \mathrm{eV}$ above the ground state minimum. This is detailed in Figure $5 \mathrm{~b}$. The $\mathrm{S}_{1}$ minimum computed by the 3-state-averaged, extended multi-state complete active space second-order perturbation method (SA3-XMS-CAS(2,2)PT2) ${ }^{93}$ on the same set of ethylene coordinates is found at $90^{\circ}$ torsion and $72^{\circ}$ pyramidalization and lies $5.46 \mathrm{eV}$ above the ground state minimum (additional information regarding the SA3-XMS-CAS(2,2)PT2 comparison can be found in the SI; these potential energy surfaces can be compared to previous results obtained with quasidegenerate multi-reference perturbation theory ${ }^{2}$ ). These results indicate that $h h$-TDA describes the potential energy surfaces reasonably well, and thus, is a promising candidate for applications in efficient nonadiabatic dynamics simulations. This finding complements earlier studies on $\mathrm{H}_{3}$ and $\mathrm{NH}_{3}$, which showed that $p p$-TDA is able to describe conical interactions. ${ }^{53}$

\section{Thymine}

The thymine molecule is a biologically relevant prototype for internal conversion dynamics between $n \pi^{*}$ and $\pi \pi^{*}$ states. At the Franck-Condon point, highly accurate coupled cluster (CC) computations predict that thymine has nearly degenerate $\mathrm{S}_{1}$ and $\mathrm{S}_{2}$ states of $n \pi^{*}$ and $\pi \pi^{*}$ character, respectively. ${ }^{86}$ Further, accurate CC-based methods predict a reaction coordinate from the FranckCondon point to the MECI between the $S_{1}$ and $S_{2}$ states without barriers or the presence of minima on the $\mathrm{S}_{2}$ state. ${ }^{86}$ At lower levels of theory - particularly those that do not include dynamic electron correlation, such as CASSCF - it is common to find a larger (greater than $1 \mathrm{eV}$ ) splitting between the $\mathrm{S}_{1}$ and $\mathrm{S}_{2}$ states at the Franck-Condon point as well as a minimum on the $\mathrm{S}_{2}$ state. ${ }^{94-95}$ It remains unclear how these properties of the thymine PES affect its photodynamics; highly efficient electronic structure methods that agree more closely with the accurate CC-based methods would be required to answer these questions. We find $h h$-TDA to be a promising candidate for use in nonadiabatic dynamics simulations of thymine.

The relative excited state energies computed with $h h$-TDA with the $\omega \operatorname{PBE}(\omega=0.2 \mathrm{au})$ functional and EOM-CCSD (see Section III) at three different thymine structures are shown in Figure 6. The vertical excitation energies to the $S_{1}$ and $S_{2}$ states are underestimated by about $0.5-$ $0.8 \mathrm{eV}$ by $h h$-TDA- $\omega \operatorname{PBE}(\omega=0.2$ a.u. $)$ compared to EOM-CCSD. The latter themselves are, however, higher by about $0.2-0.3 \mathrm{eV}$ compared to higher-level CC3 data. ${ }^{86}$ The $\mathrm{S}_{1} / \mathrm{S}_{2}$ splittings are reproduced to within $0.17-0.22 \mathrm{eV}$ by $h h$-TDA. As a result, $h h$-TDA does a particularly good job describing the relative energetics of the $S_{1}$ and $S_{2}$ states at stationary points relevant to the photodynamics of thymine. The stabilization of the $\mathrm{S}_{1}\left(n \pi^{*}\right)$ minimum is correct to $0.04 \mathrm{eV}$ 
compared to both the Franck-Condon point and $\mathrm{S}_{1} / \mathrm{S}_{2}$ MECI. Further, no minimum is present on the $S_{2}$ PES of thymine between the Franck-Condon point and $S_{1} / S_{2}$ MECI. The relative energy of that intersection with respect to the $\mathrm{S}_{2}$ energy at the Franck-Condon point is also in good agreement with EOM-CCSD. Remembering that the computational cost of $h h$-TDA scales formally as $O\left(N^{4}\right)$ but in practice as $O\left(N^{2}\right)$ with system size (see Section IV. D), while EOM-CCSD formally scales as $O\left(N^{6}\right)$, the agreement between these methods is quite remarkable. What remains to be done in future studies is to apply $h h$-TDA directly in a nonadiabatic dynamics simulation of thymine and assess the performance against experimental ultrafast spectroscopic observables.

\section{Malonaldehyde}

Malonaldehyde is a molecule that is a prototype for excited-state proton transfer, $n \pi^{*} / \pi \pi^{*}$ internal conversion dynamics, and photoisomerization. Here, we apply $h h$-TDA with the $\omega \mathrm{B} 97^{76}$ functional and compare to results obtained with multireference configuration interaction methods including single and double excitations (MRSDCI, see Figure 7). ${ }^{87}$ Again, we find good agreement in the absolute excitation energies and in the splitting between $n \pi^{*}$ and $\pi \pi^{*}$ states at the FranckCondon point. However, the relative energetics of the $S_{1}$ and $S_{2}$ states at important stationary points show some potential problems. The minimum on the $S_{1}\left(n \pi^{*}\right)$ state is a bit too stable relative to both the Franck-Condon point and the $\mathrm{S}_{0} / \mathrm{S}_{1}$ MECI. This indicates that population may become spuriously trapped on the $\mathrm{S}_{1}$ state leading to longer excited-state lifetimes and perhaps increased involvement of triplet states (in view of the El-Sayed rules and the fact that the electronic wavefunction has $n \pi^{*}$ character in the region). Also, the $\mathrm{S}_{1} / \mathrm{S}_{2}$ MECI reached by proton transfer is energetically unfavorable. This may indicate that the proton transfer reaction will play a different role in the internal conversion dynamics than MRSDCI would predict. The issues encountered by $h h$-TDA for malonaldehyde seem to be related to the restriction on the orbital space to include only one $\pi^{*}$ orbital. For this molecule, a second $\pi^{*}$ orbital may be essential to an accurate description of the photodynamics. Although we expect that malonaldehyde is not an ideal application of the $h h$-TDA method, nonadiabatic dynamics simulations, in which the molecular symmetry is lifted, would provide a more definitive assessment of the performance of $h h$-TDA for this molecule.

\section{Computational cost of hh-TDA}

In Figure 8, we report the computational time required to solve for the ground and first excited state of the Nile blue chromophore in aqueous solution using the $h h$-TDA and TDA- 
TDDFT methods with the Cartesian def2-SVP basis set and the $\omega$ PBE functional with $\omega=0.8$ a.u. The principal conclusions that should be drawn from these timings are that the $h h$-TDA and TDATDDFT methods are of similar computational cost and exhibit similar scaling behavior with system size, i.e., $\mathrm{O}\left(N^{2}\right)$ in our AO direct implementation (see below). In TDDFT, the solution of the KS equations defines the ground state, whereas in $h h$-TDA, both the ground and excited states are obtained as eigenvectors of the TDA response matrix. We find that multiplication of trial vectors against the response matrix is less expensive in the $h h$-TDA method (because there are no contributions from the Coulomb-type integrals or derivatives of the exchange-correlation potential). In cases where only one or two excited states are required, it should be expected that the cost of TDA-TDDFT and $h h$-TDA will be quite similar with the particulars of a given molecule determining which method will be faster. For example, in the present case, TDDFT required extra guess vectors in the Davidson diagonalization in order to avoid solving for higher-lying charge transfer excited states rather than the locally excited state of Nile blue.

Formally, the $h h$-TDA method appears to scale as $\mathrm{O}\left(o^{6}\right)$, where $o$ represents the number of occupied orbitals. However, since only a few electronic states are actually of interest, the full diagonalization of the response matrix can be avoided and the scaling of the method would be $\mathrm{O}\left(o^{4}\right)$ - the cost of the multiplication of a trial vector by the response matrix. However, this ignores the cost of forming the response matrix in the molecular orbital basis. In practice, our implementation of $h h$-TDA is fully AO integral direct and scales, formally, as $\mathrm{O}\left(N^{4}\right)$, where $N$ is the number of $\mathrm{AO}$ basis functions. The advantage of the $\mathrm{AO}$ formulation of $h h$-TDA is that sparsity can be exploited and the contractions between trial vectors and the response matrix scale more like $\mathrm{O}\left(N^{2}\right)$. This is clearly demonstrated in Figure 8, where the apparent quadratic scaling of the method allows us to apply it to systems with more than 2100 atoms in a polarized double- $\zeta$ basis set (roughly 18000 basis functions). It should be noted that the underlying SCF procedure costs approximately the same as the solution of the eigenvalue problem (for two states) and the SCF procedure scales slightly worse with system size.

\section{E. The unbound orbital problem and basis set requirements}

Following the remarks above on the basis set requirements for $h h$-TDA due to the $(N+2)$ electron reference, all calculations in this paper used double- and triple-zeta basis sets without diffuse functions. In order to explore the basis set dependence more thoroughly, we collect the 
lowest excitation energies computed with $h h$-TDA- $\omega$ B97X and different basis sets STO-3G, def2SVP, def2-SVPD, def2-TZVP, and def2-QZVP (the latter two without $f$ and $g$ functions) for a few molecular systems (acetamide, p-benzoquinone, butadiene, ethylene, formaldehyde, norbornadiene, uracil, and water) in Table S11 of the SI. As could be expected, these results show that excitation energies obtained with the STO-3G basis set are widely disparate from larger basis sets. However, with the exception of acetamide, the excitation energies vary by less than $0.2 \mathrm{eV}$ over the other choices of basis sets without diffuse functions. This indicates that the presence of unbound orbitals need not be a significant problem (as long as diffuse basis sets are avoided, see results for def2-SVPD).

Acetamide was among the problematic systems mentioned in Section IV.A, where DFAs with insufficient exact exchange populated an incorrect orbital in the $(\mathrm{N}+2)$-electron reference. Similar occupation problems are observed for the def 2 basis sets considered here, leading to strong basis set dependence of the excitation energy. However, this seems to be distinct from the unbound orbital problem and is instead related to the limited active space in $h h$-TDA.

Based on this assessment and the promising results obtained in this work, we therefore recommend the use of $h h$-TDA in combination with asymptotically correct range-separated hybrid DFAs and double- and triple-zeta basis sets without diffuse basis functions. The latter can be added if the excited states to be probed by $h h$-TDA are actually of Rydberg character (cf. results for water). In the future, the unbound orbital issue could be addressed by using an $N$-electron reference in the orbital generation. Such an approach was already applied in one of the early works on $p p$ RPA. ${ }^{49}$ We plan to investigate this issue in order to facilitate the routine use of $h h$-TDA in geometry optimizations and nonadiabatic dynamics simulations.

\section{Conclusions}

We have shown that the hole-hole Tamm-Dancoff approximation ( $h h$-TDA) to the particleparticle random-phase approximation ( $p p$-RPA) represents an efficient DFT-based electronic structure scheme for the electronic ground and low-lying excited states. The method shares similarities with the previously presented particle-particle ( $p p$-TDA) approach. We find some advantages of the $h$-TDA scheme, particularly in its ability to describe different low-lying excitation types $\left(n \pi^{*}, \sigma \pi^{*}\right.$, and $\left.\pi \pi^{*}\right)$. Starting from an $(N+2)$-electron reference determinant, the ground and the low-lying excited states of the $N$-electron target system are obtained by a double 
annihilation of two electrons. Since the ground and excited states are obtained from the same eigenvalue problem, static correlation cases can be handled. Dynamic correlation is included in the orbitals by virtue of the density functional. We have also introduced an alternative choice for the response kernel that differs from the functional-independent Hartree-Fock-type kernel employed in the previous works on $p p$-RPA and $p p$-TDA. ${ }^{54}$ Our choice is functional-dependent and in line with the response kernels appearing in particle-hole linear response theory.

To avoid SCF convergence problems of the $(N+2)$-electron reference, the method should be combined with range-separated density functionals that have $100 \%$ asymptotic Fock exchange. We recommend avoidance of diffuse atom-centered basis sets, since these can lead to continuumlike orbitals in the $(N+2)$-electron reference. We then do not observe any SCF convergence problems and find that the $h h$-TDA can describe low-lying excited states reasonably well at a computational complexity comparable to a configuration interaction singles (CIS) calculation. As with any density functional approximation-based method, case-specific functional assessment is recommended. The range-separation parameter and amount of short-range Fock exchange can be viewed as parameters to optimize the method performance for a specific molecule (as is often done by variation of the active space in CAS methods).

On the other hand, the main limitation of the $h h$-TDA is the restricted "virtual" orbital space, which formally consists exclusively of the lowest unoccupied orbital (with respect to the $N$ electron system). This precludes its application to systems with degenerate LUMOs, such as benzene. In spite of this shortcoming, the $h h$-TDA method is well-suited for the calculation of ground and low-lying excited states for many organic molecules. It offers an inexpensive alternative to existing and widely used CASSCF methods, with the advantage that dynamic correlation effects are included at the orbital level by means of the used density functional.

The method is implemented in the GPU-accelerated electronic structure code TeraChem. Future directions will include the computation of nonadiabatic couplings and testing the method in nonadiabatic dynamics simulations. In this context, it will be important to address the problem of potentially occupying unbound orbitals in the $(N+2)$-electron SCF calculation. Generating orbitals for an $N$-electron reference with fractional orbital occupation and using them in an $(N+2)$ electron-type $h h$-TDA procedure could remove this issue entirely, while guaranteeing stability in nonadiabatic dynamics simulations. This will be explored in future work. 
Data Availability: The data that support the findings of this study are available from the corresponding author upon reasonable request.

\section{Supplemental Material}

Detailed results on the DFA assessment (Section IV.A) and the $p p / h h$-TDA benchmarks (Section IV.B), and a potential curve of ethylene (at $90^{\circ}$ torsion) are given in a separate pdf.

\section{Acknowledgments}

This work was supported by the AMOS program of the U.S. Department of Energy, Office of Science, Basic Energy Sciences, Chemical Sciences, and Biosciences Division and by the Department of Energy, Laboratory Directed Research and Development program at SLAC National Accelerator Laboratory, under contract DE-AC02-76SF00515. TJM is a co-founder of PetaChem, LLC. CB thanks the German National Academy of Sciences Leopoldina for support through the Leopoldina Fellowship Program (Project No. LPDS 2018-09). The authors thank Deniz Tuna for helpful discussions and for early testing of the method. 


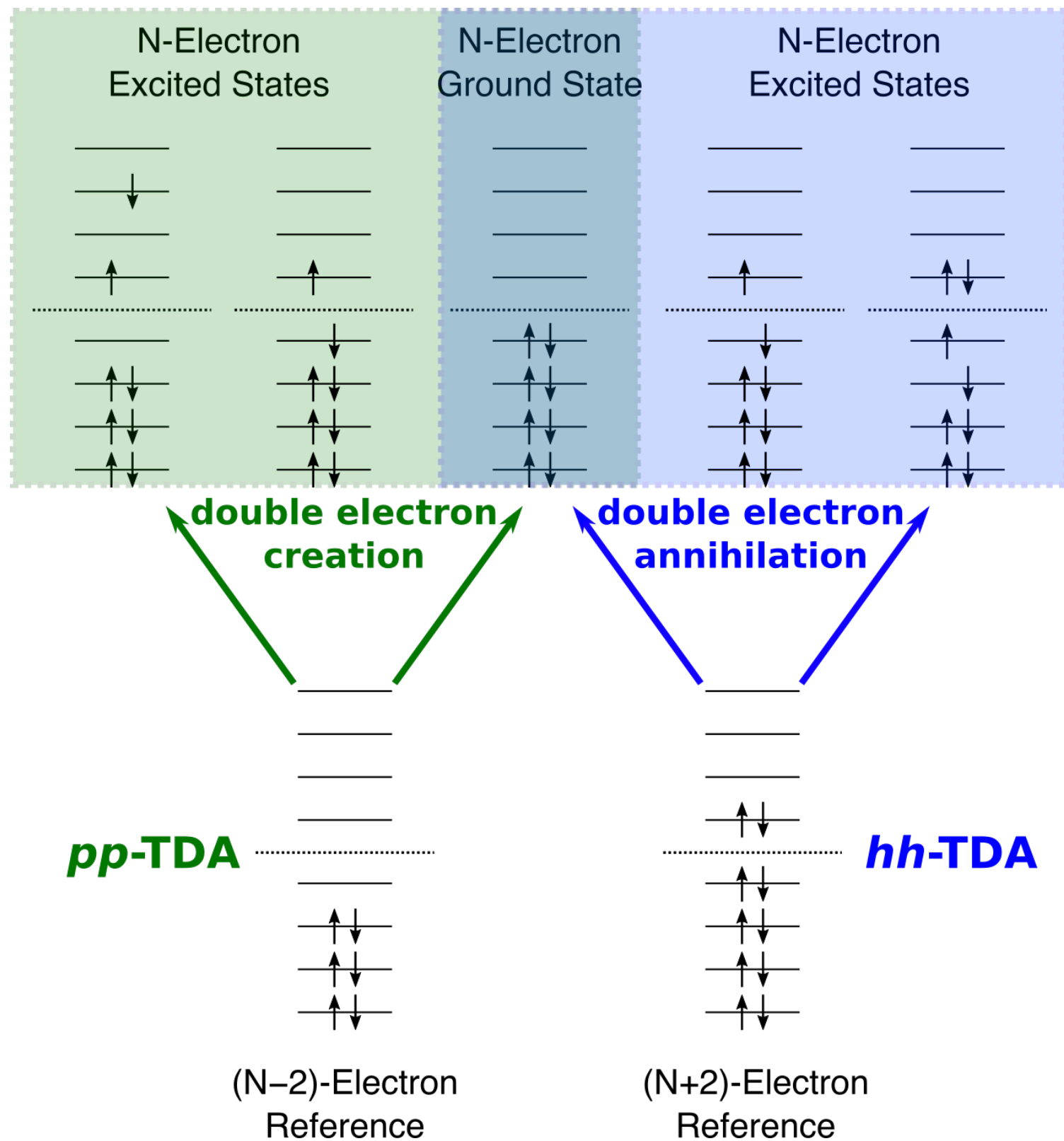

Figure 1: Graphical representation of the $p p$-TDA (left) and $h h$-TDA (right) methods to obtain the $\mathrm{N}$-electron ground state and excited states. In the $p p$-TDA case (left), the orbitals are obtained from a SCF calculation on the ( $N-2$ )-electron system. Addition (green) of two electrons into the virtual space recovers the $N$-electron ground and excited states. The closed-shell $N$-electron ground state obtained from $p p$-TDA is dominated by the configuration that corresponds to a double creation in the LUMO (with respect to the ( $N-2)$-electron system). In the $h h$-TDA case (right), the orbitals are obtained from a SCF calculation on the $(N+2)$-electron system. Annihilation (blue) of the two electrons in the occupied orbital space then recovers the $N$-electron ground and excited states. Here, the $\mathrm{N}$-electron ground state is dominated by the configuration that corresponds to a double annihilation in the HOMO (with respect to the $(N+2)$-electron reference). 


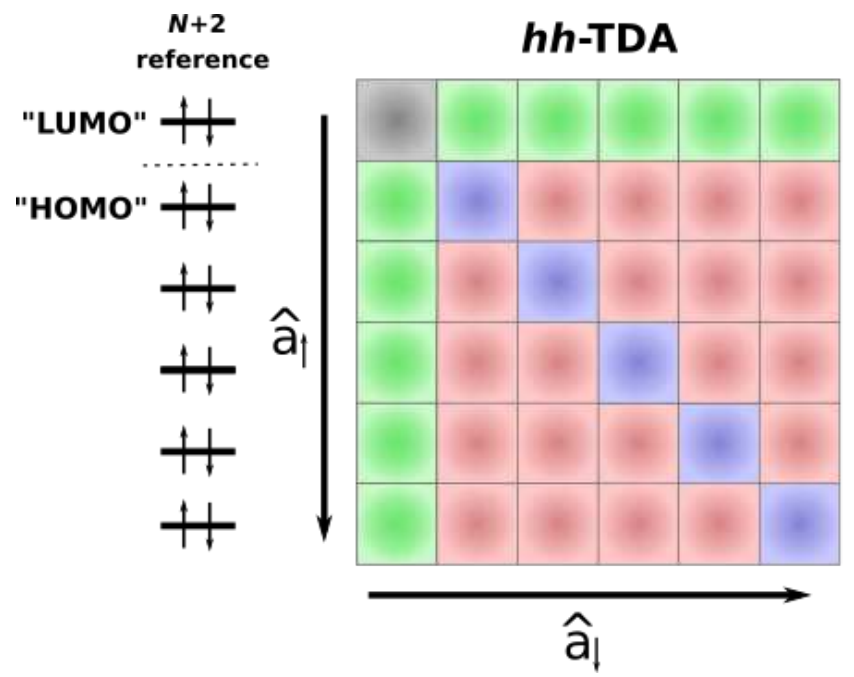

\section{$\mathrm{N}$-electron configurations}

closed-shell ground state determinant

open-shell singly excited determinant

closed-shell doubly excited determinant

open-shell doubly excited determinant

Figure 2: Schematic representation of the excitation types generated in $h h$-TDA with respect to the $N$-electron ground state. The "LUMO" and "HOMO" orbitals define this $N$-electron state.

(a)

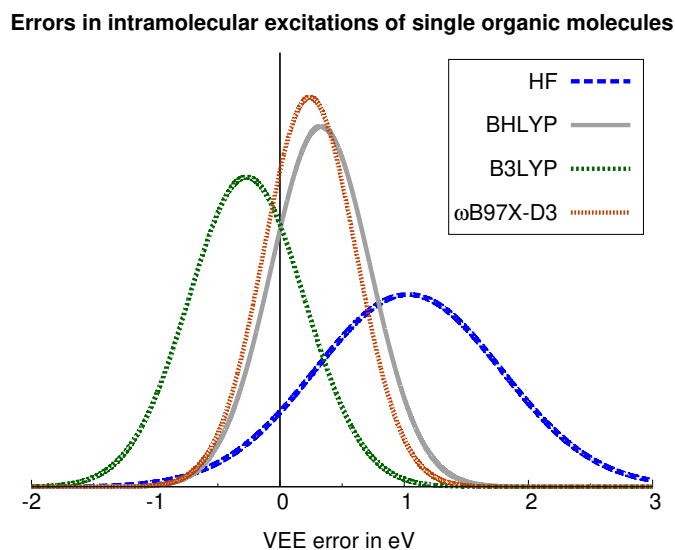

(b)

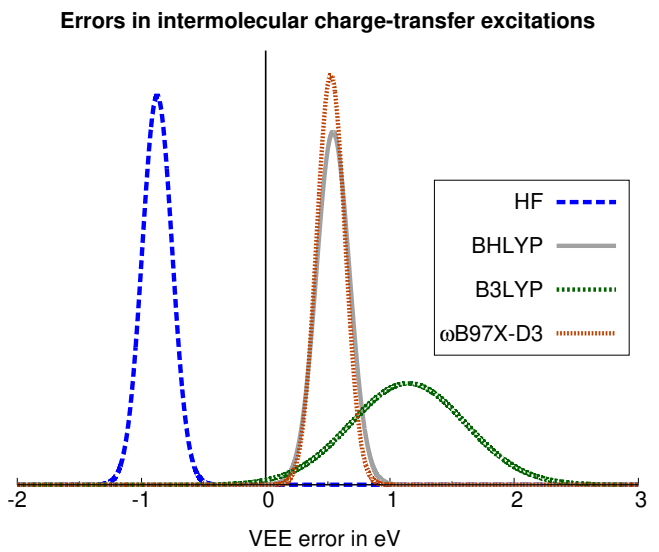

Figure 3: Gaussian error distribution functions for $h h$-TDA with different density functional approximations in the calculation of vertical excitation energies (VEEs). The spherical def2$\mathrm{SV}(\mathrm{P})^{78-79}$ basis set and the LR kernel in the $h h$-TDA calculation have been used throughout. The centers of the Gaussians correspond to the mean deviation (MD), whereas the width of the Gaussian corresponds to the standard deviation (SD), both in $\mathrm{eV}$. (a) Lowest vertical excitations of single molecules with no or little intramolecular CT character. The individual MDs and SDs in $\mathrm{eV}$ are: B3LYP $(-0.27,0.47)$, BHLYP $(0.33,0.40)$, HF $(1.03,0.75)$, and $\omega B 97 X-D 3(0.24,0.37)$, with $N=27$. (b) Intermolecular CT excitations of organic bimolecular complexes. The individual MDs and SDs in eV are: B3LYP $(1.15,0.46)$, BHLYP $(0.54,0.13), \operatorname{HF}(-0.87,0.12)$, and $\omega B 97 X-$ D3 $(0.52,0.11)$, with $N=6$. See SI for details on the benchmark sets and results with other functionals. 


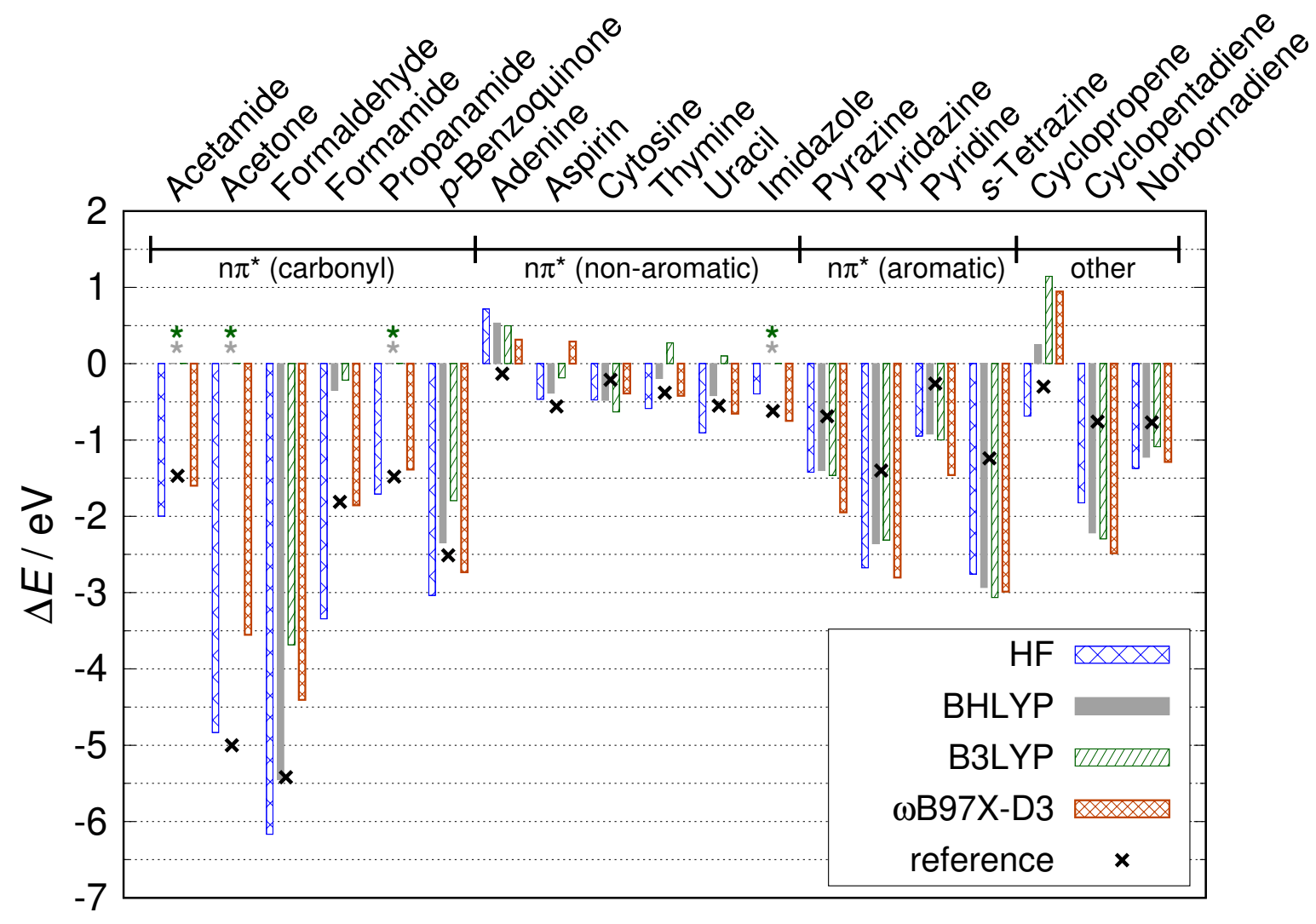

Figure 4: Energetic splitting between a low-lying $\pi \pi^{*}$ and another (usually $n \pi^{*}$ ) excited state. The excitation energies were computed at the $h h$-TDA level of theory (LR kernel) employing the spherical def2-SV(P) basis set. A $\pi$-system is classified as "aromatic," if a near-degeneracy of the $N$-electron system LUMO orbital can be expected, due to the symmetry of the molecule (cf. Frost circle representation). An asterisk marks missing data, due to an incorrect LUMO (with respect to the $N$-electron system) occupation in the $(N+2)$-electron SCF calculation. The reference values are taken from Ref. ${ }^{81}$ (aspirin from Ref. ${ }^{90}$ ). The respective state symmetries are given in the SI. 
Table 1: Statistical data for excitation energies (in $\mathrm{eV}$ ) computed with $h h$-TDA and $p p$-TDA using either the linear response (LR) type response kernel (this work) or the Hartree-Fock (HF) response kernel employed by Yang and coworkers. Particle-hole TD-DFT (Tamm-Dancoff approximated, TDA) calculations and EOM-CCSD calculations are provided for comparison. All calculations use the spherical TZVP basis set. We chose DFAs which we expect to perform best for both schemes, i.e., a range-separated one for $h h$-TDA and a GGA for $p p$-TDA. The structures and reference values are taken from Ref. ${ }^{81}$. If states could not be described by a method, they were discarded from the statistical analysis. The preceding PBE SCF calculations for the $(N-2)$-electron reference failed to converge for eight systems, precluding a total of 23 states to be considered for $p p$-TDAPBE (see SI for details).

\begin{tabular}{|c|c|c|c|c|c|c|}
\hline & EOM-CCSD & $T D A-\omega B 97 X$ & $\begin{array}{r}h h-T D A-\omega B 97 X \\
(L R) \\
\end{array}$ & $\begin{array}{r}h h-T D A-\omega B 97 X \\
(H F)\end{array}$ & $p p-T D A-P B E(L R)$ & $p p-T D A-P B E(H F)$ \\
\hline $\mathbf{M D}^{\mathrm{a}}$ & 0.43 & 0.38 & 0.36 & 0.43 & -0.25 & -0.30 \\
\hline$S T D^{\mathrm{b}}$ & 0.32 & 0.30 & 0.59 & 0.69 & 1.14 & 0.69 \\
\hline$M A D^{c}$ & 0.44 & 0.40 & 0.54 & 0.67 & 0.98 & 0.59 \\
\hline States $^{d}$ & 51 & 48 & 48 & 48 & 18 & 18 \\
\hline
\end{tabular}

${ }^{a}$ Mean deviation. ${ }^{b}$ Standard deviation. ${ }^{c}$ Mean absolute deviation. ${ }^{d}$ Number of states included in the statistical data. 

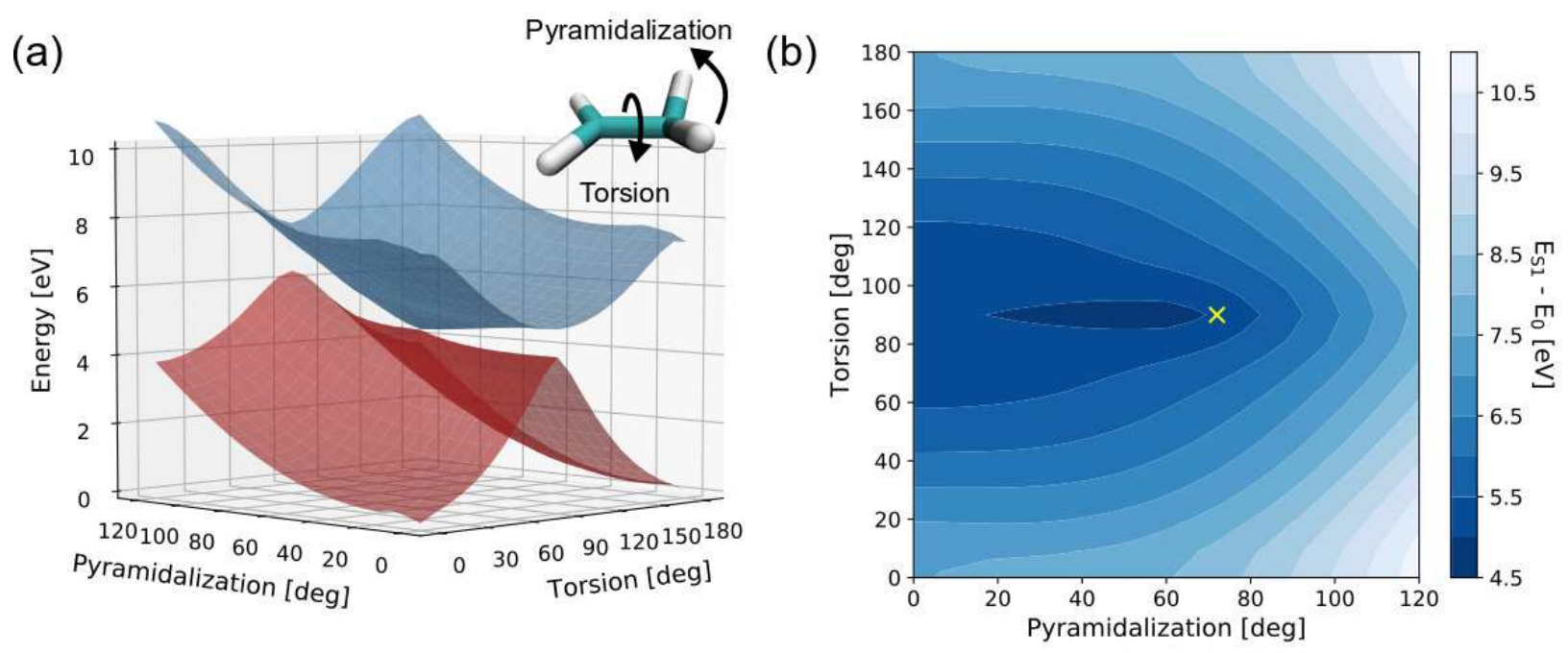

Figure 5: (a) Global features of the $\mathrm{S}_{0}$ ground (red) and $\mathrm{S}_{1}$ excited (blue) state PESs of ethylene computed at the $h h$-TDA- $\omega \mathrm{B} 97 \mathrm{X} / \mathrm{def} 2-\mathrm{SVP}$ level in a rigid scan over the branching plane coordinates of pyramidalization and torsion (visualized in the inset). The linear response-type kernel has been employed. $h h$-TDA describes the $\mathrm{S}_{0} / \mathrm{S}_{1}$ degeneracy in ethylene, as demonstrated here with the appearance of the double cone feature. Ethylene reaches the conical intersection geometry once distorted to 90 degrees torsion and at 60 degrees pyramidalization. This point coincides with the predicted $\mathrm{S}_{1}$ minimum, lying $4.76 \mathrm{eV}$ above the ground state minimum. (b) The $\mathrm{S}_{1}$ PES from (a) represented as a contour plot. The shape of the $\mathrm{S}_{1}$ PES is relatively well-described by $h h$-TDA- $\omega$ B97X compared to previously reported quasidegenerate multi-reference perturbation theory results. ${ }^{2}$ The $\mathrm{S}_{1}$ minimum appears at a simultaneously twisted and pyramidalized geometry. For comparison, the location of the $\mathrm{S}_{1}$ minimum obtained from SA3XMS-CAS(2,2)PT2 (see SI for details) that lies $5.46 \mathrm{eV}$ above the $\mathrm{S}_{0}$ ground state is marked by a light-yellow X. 


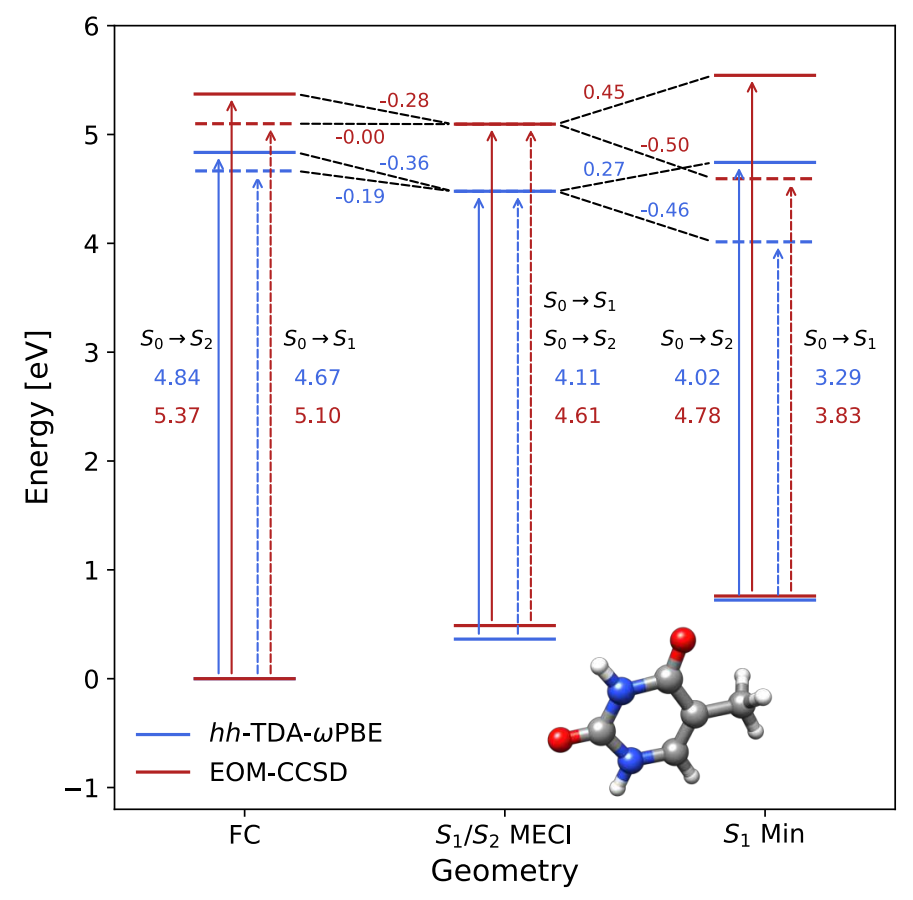

Figure 6: Relative energies (in $\mathrm{eV}$ ) of the lowest three singlet electronic states of thymine computed at the $h h-\mathrm{TDA}-\omega \mathrm{PBE}(\omega=0.2$ a.u. $) / 6-31 \mathrm{G}^{* *}$ (blue) and EOM-CCSD/aug-cc-pVDZ (red) levels of theory. Energies are computed at three critical points relevant to the excited state dynamics of thymine: the $\mathrm{S}_{0}$ minimum $(\mathrm{FC})$, the minimum energy conical intersection between the $\mathrm{S}_{1}$ and $\mathrm{S}_{2}$ states $\left(\mathrm{S}_{1} / \mathrm{S}_{2} \mathrm{MECI}\right)$, and the $\mathrm{S}_{1}$ minimum $\left(\mathrm{S}_{1} \mathrm{~min}\right)$. The arrows indicate vertical excitations to $S_{2}$ (solid) and $S_{1}$ (dashed). Geometries are optimized for $h h$-TDA and taken from Ref. ${ }^{86}$ for EOM-CCSD (see Section III for technical details). All energies are given relative to the respective $\mathrm{S}_{0}$ energy at the Franck-Condon point. Higher level vertical excitation energies at the CC3 level of theory are 0.20-0.26 eV lower compared to our EOM-CCSD/aug-cc-pVDZ (cf. Ref. $\left.{ }^{86}\right)$, and thus in better agreement with the $h h-\mathrm{TDA}-\omega \mathrm{PBE}(\omega=0.2$ a.u. $) / 6-31 \mathrm{G}^{* *}$ results. 


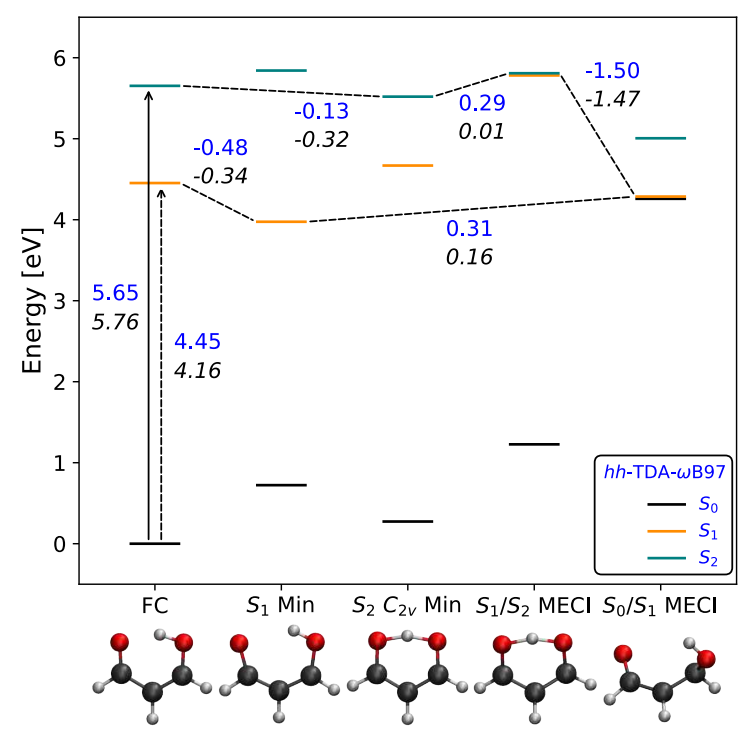

Figure 7: Relative energies (in eV) of the lowest three singlet electronic states of malonaldehyde for critical geometries computed at the $h h$-TDA- $\omega$ B $97 / 6-31 \mathrm{G}^{* *}$ (blue normal print) and MRSDCI/6-31G*//SA3-CAS(4,4)SCF/6-31G* (black italics, taken from Ref. ${ }^{87}$ ) levels of theory. In the latter, all configurations generated in a $(4,4)$ active space served as references in the MRSDCI calculations. The Franck-Condon, $\mathrm{S}_{1}$ minimum, and $\mathrm{S}_{0} / \mathrm{S}_{1}$ minimum energy conical intersection geometries are optimized without constraints. The $\mathrm{S}_{2} C_{2 v}$ minimum and $\mathrm{S}_{1} / \mathrm{S}_{2}$ MECI are minima in the $C_{2 v}$ and $C_{s}$ point groups, respectively. Only the energy levels computed at the $h h$-TDA- $\omega$ B97/6-31G** level of theory are plotted and the vertical excitations at the FranckCondon point to $S_{2}$ and $S_{1}$ are illustrated as solid and dashed arrows, respectively. 


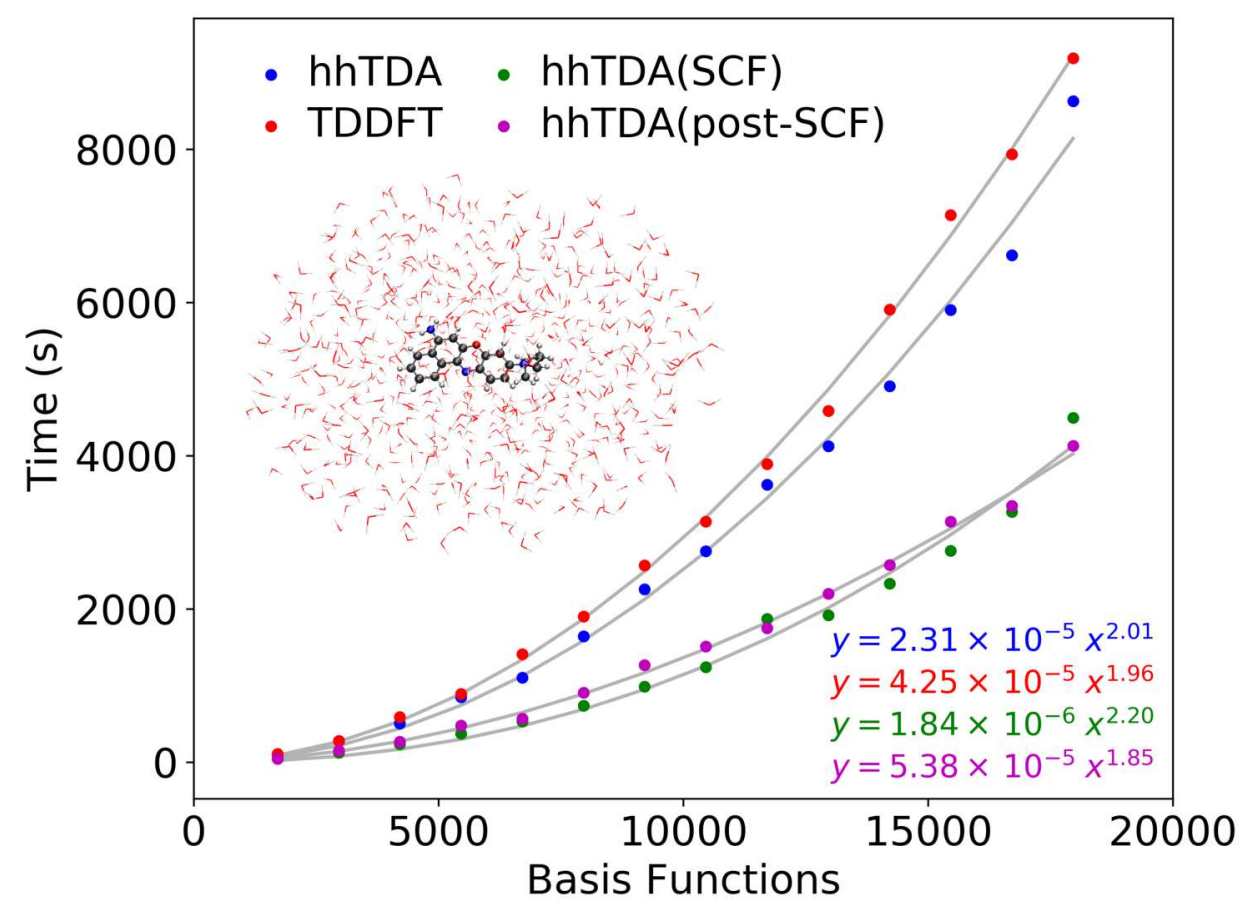

Figure 8: Timings of $h h$-TDA/def2-SVP and TDA-TDDFT/def2-SVP computations of the ground and first excited state of Nile blue including microsolvation by 50 to 700 water molecules. The $h h$ TDA/def2-SVP computation is decomposed into the time spent in the KS SCF for the $(N+2)-$ electron system and the "post-SCF" portion of the computation that comprises the Davidson diagonalization to obtain the lowest two eigenpairs as defined in Eq. 18. The largest system considered contained more than 2100 atoms and nearly 18000 basis functions. The timings were performed using 8 NVIDIA V100 GPUs and 8 Intel Xeon E5-2698 CPU cores clocked at 2.2 GHz. Best fit power series are given for each of the methods. For both methods, the $\omega$ PBE functional with a range-separation parameter of 0.8 a.u. was used. 


\section{References}

1. Dreuw, A.; Head-Gordon, M., Single-Reference ab Initio Methods for the Calculation of Excited States of Large Molecules. Chem. Rev. 2005, 105, 4009-4037.

2. Levine, B. G.; Ko, C.; Quenneville, J.; Martínez, T. J., Conical intersections and double excitations in time-dependent density functional theory. Mol. Phys. 2006, 104, 1039-1051.

3. Roos, B. O., The complete active space SCF method in a fock-matrix-based super-CI formulation. Int. J. Quantum Chem. 1980, 18, 175-189.

4. Roos, B. O., Theoretical studies of electronically excited states of molecular systems using multiconfigurational perturbation theory. Acc. Chem. Res. 1999, 32, 137-144.

5. Werner, H. J.; Knowles, P. J., An Efficient Internally Contracted Multiconfiguration Reference Configuration-Interaction Method. J. Chem. Phys. 1988, 89, 5803-5814.

6. Coe, J. D.; Levine, B. G.; Martinez, T. J., Ab Initio Molecular Dynamics of Excited State Intramolecular Proton Transfer Using Multireference Perturbation Theory. J. Phys. Chem. A 2007, $111,11302-11310$.

7. Tao, H.; Levine, B. G.; Martinez, T. J., Ab Initio Multiple Spawning Dynamics Using MultiState Second-Order Perturbation Theory. J. Phys. Chem. A 2009, 113, 13656-13662.

8. Tao, H.; Allison, T. K.; Wright, T. W.; Stooke, A. M.; Khurmi, C.; van Tilborg, J.; Liu, Y.; Falcone, R. W.; Belkacem, A.; Martinez, T. J., Ultrafast internal conversion in ethylene. I. The excited state lifetime. J. Chem. Phys. 2011, 134, 244306.

9. Mori, T.; Glover, W. J.; Schuurman, M. S.; Martinez, T. J., Role of Rydberg States in the Photochemical Dynamics of Ethylene. J. Phys. Chem. A 2012, 116, 2808-2818.

10. Liu, L.; Liu, J.; Martinez, T. J., Dynamical Correlation Effects on Photoisomerization: Ab Initio Multiple Spawning Dynamics with MS-CASPT2 for a Model trans-Protonated Schiff Base. J. Phys. Chem. B 2016, 120, 1940-1949.

11. Park, J. W.; Shiozaki, T., On-the-fly CASPT2 Surface-Hopping Dynamics. J. Chem. Theo. Comp. 2017, 13, 3676-3683.

12. Glover, W. J.; Mori, T.; Schuurman, M. S.; Boguslavskiy, A. E.; Schalk, O.; Stolow, A.; Martinez, T. J., Excited state non-adiabatic dynamics of the smallest polyene, trans 1,3-butadiene. II. Ab initio multiple spawning simulations. J. Chem. Phys. 2018, 148, 164303.

13. Snyder, J. W.; Parrish, R. M.; Martínez, T. J., $\alpha$-CASSCF: An Efficient, Empirical Correction for SA-CASSCF To Closely Approximate MS-CASPT2 Potential Energy Surfaces. J. Phys. Chem. Lett. 2017, 8, 2432-2437.

14. Koslowski, A.; Beck, M. E.; Thiel, W., Implementation of a general multireference configuration interaction procedure with analytic gradients in a semiempirical context using the graphical unitary group approach. J. Comp. Chem. 2003, 24, 714-726.

15. Toniolo, A.; Thompson, A. L.; Martinez, T. J., Excited state direct dynamics of benzene with reparameterized multi-reference semiempirical configuration interaction methods. Chem. Phys. 2004, 304, 133-145.

16. Toniolo, A.; Granucci, G.; Inglese, S.; Persico, M., Theoretical study of the photodissociation dynamics of ClOOCl. Phys. Chem. Chem. Phys. 2001, 3, 4266-4279.

17. Sastry, K.; Johnson, D. D.; Thompson, A. L.; Goldberg, D. E.; Martinez, T. J.; Leiding, J.; Owens, J., Optimization of Semiempirical Quantum Chemistry Methods via Multiobjective Genetic Algorithms: Accurate Photodynamics for Larger Molecules and Longer Time Scales. Mat. Manu. Proc. 2007, 22, 553-561. 
18. Ghosh, S.; Verma, P.; Cramer, C. J.; Gagliardi, L.; Truhlar, D. G., Combining Wave Function Methods with Density Functional Theory for Excited States. Chem. Rev. 2018, 118, 7249-7292.

19. Pijeau, S.; Hohenstein, E. G., Improved complete active space configuration interaction energies with a simple correction from density functional theory. J. Chem. Theo. Comp. 2017, 13, $1130-1146$.

20. Miehlich, B.; Stoll, H.; Savin, A., A correlation-energy density functional for multideterminantal wavefunctions. Mol. Phys. 1997, 91, 527-536.

21. Gutle, C.; Savin, A., Orbital spaces and density-functional theory. Phys. Rev. A 2007, 75.

22. Gagliardi, L.; Truhlar, D. G.; Li Manni, G.; Carlson, R. K.; Hoyer, C. E.; Bao, J. W. L., Multiconfiguration Pair-Density Functional Theory: A New Way To Treat Strongly Correlated Systems. Acc. Chem. Res. 2017, 50, 66-73.

23. Sand, A. M.; Hoyer, C. E.; Truhlar, D. G.; Gagliardi, L., State-interaction pair-density functional theory. J. Chem. Phys. 2018, 149.

24. Li, S. L.; Marenich, A. V.; Xu, X.; Truhlar, D. G., Configuration Interaction-Corrected TammDancoff Approximation: A Time-Dependent Density Functional Method with the Correct Dimensionality of Conical Intersections. J. Phys. Chem. Lett. 2014, 5, 322-328.

25. Teh, H.-H.; Subotnik, J. E., The Simplest Possible Approach for Simulating S0-S1 Conical Intersections with DFT/TDDFT: Adding One Doubly Excited Configuration. J. Phys. Chem. Lett. 2019, 10, 3426-3432.

26. Grimme, S.; Waletzke, M., A combination of Kohn-Sham density functional theory and multireference configuration interaction methods. J. Chem. Phys. 1999, 111, 5645-5655.

27. Marian, C. M.; Heil, A.; Kleinschmidt, M., The DFT/MRCI method. WIREs Comp. Mol. Sci. 2019, 9 .

28. Shu, Y.; Parker, K. A.; Truhlar, D. G., Dual-Functional Tamm-Dancoff Approximation: A Convenient Density Functional Method that Correctly Describes S1/S0 Conical Intersections. $J$. Phys. Chem. Lett. 2017, 8, 2107-2112.

29. Krylov, A. I.; Shao, Y.; Head-Gordon, M., The spin-flip approach within time-dependent density functional theory: Theory and applications to diradicals. J. Chem. Phys. 2003, 118, 48074818 .

30. Sears, J. S.; Sherrill, C. D.; Krylov, A. I., A spin-complete version of the spin-flip approach to bond breaking: What is the impact of obtaining spin eigenfunctions? J. Chem. Phys. 2003, 118, 9084-9094.

31. Casanova, D.; Head-Gordon, M., The spin-flip extended single excitation configuration interaction method. J. Chem. Phys. 2008, 129.

32. Zhang, X.; Herbert, J. M., Spin-flip, tensor equation-of-motion configuration interaction with a density-functional correction: A spin-complete method for exploring excited-state potential energy surfaces. J. Chem. Phys. 2015, 143.

33. Filatov, M., Spin-restricted ensemble-referenced Kohn-Sham method: basic principles and application to strongly correlated ground and excited states of molecules. WIREs Comp. Mol. Sci. 2015, 5, 146-167.

34. Filatov, M.; Liu, F.; Martínez, T. J., Analytical derivatives of the individual state energies in ensemble density functional theory method. I. General formalism. J. Chem. Phys. 2017, 147, 034113.

35. Liu, F.; Filatov, M.; Martínez, T. J., Analytical Derivatives of the Individual State Energies in Ensemble Density Functional Theory Method: II. Implementation on Graphical Processing Units (GPUs). ChemRxiv 2019, DOI: 10.26434/chemrxiv.7985657. 
36. Peng, D.; Steinmann, S. N.; Aggelen, H. v.; Yang, W., Equivalence of particle-particle random phase approximation correlation energy and ladder-coupled-cluster doubles. J. Chem. Phys. 2013, 139, 104112.

37. van Aggelen, H.; Yang, Y.; Yang, W., Exchange-correlation energy from pairing matrix fluctuation and the particle-particle random-phase approximation. Phys. Rev. A 2013, 88, 030501. 38. van Aggelen, H.; Yang, Y.; Yang, W., Exchange-correlation energy from pairing matrix fluctuation and the particle-particle random phase approximation. J. Chem. Phys. 2014, 140, 18A511.

39. Yang, Y.; van Aggelen, H.; Steinmann, S. N.; Peng, D.; Yang, W., Benchmark tests and spin adaptation for the particle-particle random phase approximation. J. Chem. Phys. 2013, 139, 174110.

40. Shenvi, N.; van Aggelen, H.; Yang, Y.; Yang, W., Tensor hypercontracted ppRPA: Reducing the cost of the particle-particle random phase approximation from $\mathrm{O}(\mathrm{r} 6)$ to $\mathrm{O}(\mathrm{r} 4)$. J. Chem. Phys. 2014, 141, 024119.

41. Scuseria, G. E.; Henderson, T. M.; Bulik, I. W., Particle-particle and quasiparticle random phase approximations: Connections to coupled cluster theory. J. Chem. Phys. 2013, 139, 104113. 42. Scuseria, G. E.; Henderson, T. M.; Sorensen, D. C., The ground state correlation energy of the random phase approximation from a ring coupled cluster doubles approach. J. Chem. Phys. 2008, 129, 231101.

43. Al-Saadon, R.; Sutton, C.; Yang, W., Accurate Treatment of Charge-Transfer Excitations and Thermally Activated Delayed Fluorescence Using the Particle-Particle Random Phase Approximation. J. Chem. Theo. Comp. 2018, 14, 3196-3204.

44. Jin, Y.; Yang, Y.; Zhang, D.; Peng, D.; Yang, W., Excitation energies from particle-particle random phase approximation with accurate optimized effective potentials. J. Chem. Phys. 2017, $147,134105$.

45. Yang, Y.; Peng, D.; Lu, J.; Yang, W., Excitation energies from particle-particle random phase approximation: Davidson algorithm and benchmark studies. J. Chem. Phys. 2014, 141, 124104.

46. Sutton, C.; Yang, Y.; Zhang, D.; Yang, W., Single, Double Electronic Excitations and Exciton Effective Conjugation Lengths in $\pi$-Conjugated Systems. J. Phys. Chem. Lett. 2018, 9, 4029-4036. 47. Yang, Y.; Dominguez, A.; Zhang, D.; Lutsker, V.; Niehaus, T. A.; Frauenheim, T.; Yang, W., Charge transfer excitations from particle-particle random phase approximation-Opportunities and challenges arising from two-electron deficient systems. J. Chem. Phys. 2017, 146, 124104. 48. Yang, Y.; Peng, D.; Davidson, E. R.; Yang, W., Singlet-Triplet Energy Gaps for Diradicals from Particle-Particle Random Phase Approximation. J. Phys. Chem. A 2015, 119, 4923-4932. 49. Yang, Y.; van Aggelen, H.; Yang, W., Double, Rydberg and charge transfer excitations from pairing matrix fluctuation and particle-particle random phase approximation. J. Chem. Phys. 2013, $139,224105$.

50. Zhang, D.; Peng, D.; Zhang, P.; Yang, W., Analytic gradients, geometry optimization and excited state potential energy surfaces from the particle-particle random phase approximation. Phys. Chem. Chem. Phys. 2015, 17, 1025-1038.

51. Pinter, B.; Al-Saadon, R.; Chen, Z.; Yang, W., Spin-state energetics of iron(II) porphyrin from the particle-particle random phase approximation. Eur. Phys. J. B 2018, 91, 270.

52. Yang, Y.; Davidson, E. R.; Yang, W., Nature of ground and electronic excited states of higher acenes. Proc. Natl. Acad. Sci. 2016, 113, E5098.

53. Yang, Y.; Shen, L.; Zhang, D.; Yang, W., Conical Intersections from Particle-Particle Random Phase and Tamm-Dancoff Approximations. J. Phys. Chem. Lett. 2016, 7, 2407-2411. 
54. Peng, D.; van Aggelen, H.; Yang, Y.; Yang, W., Linear-response time-dependent densityfunctional theory with pairing fields. J. Chem. Phys. 2014, 140, 18A522.

55. Oliveira, L. N.; Gross, E. K. U.; Kohn, W., Density-Functional Theory for Superconductors. Phys. Rev. Lett. 1988, 60, 2430-2433.

56. Gross, E. K. U.; Kurth, S., Density-functional theory of the superconducting state. Int. J. Quantum Chem. 1991, 40, 289-297.

57. Lüders, M.; Marques, M. A. L.; Lathiotakis, N. N.; Floris, A.; Profeta, G.; Fast, L.; Continenza, A.; Massidda, S.; Gross, E. K. U., Ab initio theory of superconductivity. I. Density functional formalism and approximate functionals. Phys. Rev. B 2005, 72, 024545.

58. Casida, M. E., Time-Dependent Density Functional Response Theory for Molecules in: Recent Advances in Density Functional Methods, Vol. 1 (D. P. Chong, Ed.). World Scientific: Singapore, 1995.

59. Kohn, A.; Tajti, A., Can coupled-cluster theory treat conical intersections? J. Chem. Phys. 2007, 127, 044105.

60. Kjonstad, E. F.; Myhre, R. H.; Martinez, T. J.; Koch, H., Crossing conditions in coupled cluster theory. J. Chem. Phys. 2017, 147, 164105.

61. Rienstra-Kiracofe, J. C.; Tschumper, G. S.; Schaefer, H. F.; Nandi, S.; Ellison, G. B., Atomic and Molecular Electron Affinities: Photoelectron Experiments and Theoretical Computations. Chem. Rev. 2002, 102, 231-282.

62. Perdew, J. P.; Yang, W.; Burke, K.; Yang, Z.; Gross, E. K. U.; Scheffler, M.; Scuseria, G. E.; Henderson, T. M.; Zhang, I. Y.; Ruzsinszky, A.; Peng, H.; Sun, J.; Trushin, E.; Görling, A., Understanding band gaps of solids in generalized Kohn-Sham theory. Proc. Natl. Acad. Sci. 2017, $114,2801$.

63. Baerends, E. J.; Gritsenko, O. V.; van Meer, R., The Kohn-Sham gap, the fundamental gap and the optical gap: the physical meaning of occupied and virtual Kohn-Sham orbital energies. Phys. Chem. Chem. Phys. 2013, 15, 16408-16425.

64. Ufimtsev, I. S.; Martínez, T. J., Quantum Chemistry on Graphical Processing Units. 1. Strategies for Two-Electron Integral Evaluation. J. Chem. Theo. Comp. 2008, 4, 222-231.

65. Ufimtsev, I. S.; Martinez, T. J., Quantum Chemistry on Graphical Processing Units. 2. Direct Self-Consistent-Field Implementation. J. Chem. Theo. Comp. 2009, 5, 1004-1015.

66. Becke, A. D., Density-functional exchange-energy approximation with correct asymptotic behaviour. Phys. Rev. A 1988, 38, 3098-3100.

67. Lee, C.; Yang, W.; Parr, R. G., Development of the Colle-Salvetti correlation-energy formula into a functional of the electron density. Phys. Rev. B 1988, 37, 785-789.

68. Becke, A. D., Density-functional thermochemistry. III. The role of exact exchange. J. Chem. Phys. 1993, 98, 5648-5652.

69. Stephens, P. J.; Devlin, F. J.; Chabalowski, C. F.; Frisch, M. J., Ab Initio Calculation of Vibrational Absorption and Circular Dichroism Spectra Using Density Functional Force Fields. $J$. Phys. Chem. 1994, 98, 11623-11627.

70. Perdew, J. P.; Burke, K.; Ernzerhof, M., Generalized Gradient Approximation Made Simple. Phys. Rev. Lett. 1996, 77, 3865-3868.

71. Adamo, C.; Barone, V., Toward reliable density functional methods without adjustable parameters: The PBE0 model. J. Chem. Phys. 1999, 110, 6158-6170.

72. Becke, A. D., A new mixing of Hartree-Fock and local density-functional theories. J. Chem. Phys. 1993, 98, 1372-1377. 
73. Yanai, T.; Tew, D. P.; Handy, N. C., A new hybrid exchange-correlation functional using the Coulomb-attenuating method (CAM-B3LYP). Chem. Phys. Lett 2004, 393, 51-57.

74. Rohrdanz, M. A.; Martins, K. M.; Herbert, J. M., A long-range-corrected density functional that performs well for both ground-state properties and time-dependent density functional theory excitation energies, including charge-transfer excited states. J. Chem. Phys. 2009, 130, 054112.

75. Becke, A. D., Density-functional thermochemistry. V. Systematic optimization of exchangecorrelation functionals. J. Chem. Phys. 1997, 107, 8554-8560.

76. Chai, J.-D.; Head-Gordon, M., Systematic optimization of long-range corrected hybrid density functionals. J. Chem. Phys. 2008, $128,084106$.

77. Lin, Y.-S.; Li, G.-D.; Mao, S.-P.; Chai, J.-D., Long-Range Corrected Hybrid Density Functionals with Improved Dispersion Corrections. J. Chem. Theo. Comp. 2013, 9, 263-272.

78. Schäfer, A.; Horn, H.; Ahlrichs, R., Fully optimized contracted Gaussian basis sets for atoms Li to Kr. J. Chem. Phys. 1992, 97, 2571-2577.

79. Weigend, F.; Ahlrichs, R., Balanced basis sets of split valence, triple zeta valence and quadruple zeta valence quality for $\mathrm{H}$ to $\mathrm{Rn}$ : Design and assessment of accuracy. Phys. Chem. Chem. Phys. 2005, 7, 3297-3305.

80. Schäfer, A.; Huber, C.; Ahlrichs, R., Fully optimized contracted Gaussian basis sets of triple zeta valence quality for atoms Li to Kr. J. Chem. Phys. 1994, 100, 5829-5835.

81. Schreiber, M.; Silva-Junior, M. R.; Sauer, S. P. A.; Thiel, W., Benchmarks for electronically excited states: CASPT2, CC2, CCSD, and CC3. J. Chem. Phys. 2008, 128, 134110.

82. Shao, Y.; Gan, Z.; Epifanovsky, E.; Gilbert, A. T. B.; Wormit, M.; Kussmann, J.; Lange, A. W.; Behn, A.; Deng, J.; Feng, X.; Ghosh, D.; Goldey, M.; Horn, P. R.; Jacobson, L. D.; Kaliman, I.; Khaliullin, R. Z.; Kuś, T.; Landau, A.; Liu, J.; Proynov, E. I.; Rhee, Y. M.; Richard, R. M.; Rohrdanz, M. A.; Steele, R. P.; Sundstrom, E. J.; Woodcock, H. L.; Zimmerman, P. M.; Zuev, D.; Albrecht, B.; Alguire, E.; Austin, B.; Beran, G. J. O.; Bernard, Y. A.; Berquist, E.; Brandhorst, K.; Bravaya, K. B.; Brown, S. T.; Casanova, D.; Chang, C.-M.; Chen, Y.; Chien, S. H.; Closser, K. D.; Crittenden, D. L.; Diedenhofen, M.; DiStasio, R. A.; Do, H.; Dutoi, A. D.; Edgar, R. G.; Fatehi, S.; Fusti-Molnar, L.; Ghysels, A.; Golubeva-Zadorozhnaya, A.; Gomes, J.; Hanson-Heine, M. W. D.; Harbach, P. H. P.; Hauser, A. W.; Hohenstein, E. G.; Holden, Z. C.; Jagau, T.-C.; Ji, H.; Kaduk, B.; Khistyaev, K.; Kim, J.; Kim, J.; King, R. A.; Klunzinger, P.; Kosenkov, D.; Kowalczyk, T.; Krauter, C. M.; Lao, K. U.; Laurent, A. D.; Lawler, K. V.; Levchenko, S. V.; Lin, C. Y.; Liu, F.; Livshits, E.; Lochan, R. C.; Luenser, A.; Manohar, P.; Manzer, S. F.; Mao, S.-P.; Mardirossian, N.; Marenich, A. V.; Maurer, S. A.; Mayhall, N. J.; Neuscamman, E.; Oana, C. M.; OlivaresAmaya, R.; O’Neill, D. P.; Parkhill, J. A.; Perrine, T. M.; Peverati, R.; Prociuk, A.; Rehn, D. R.; Rosta, E.; Russ, N. J.; Sharada, S. M.; Sharma, S.; Small, D. W.; Sodt, A.; Stein, T.; Stück, D.; Su, Y.-C.; Thom, A. J. W.; Tsuchimochi, T.; Vanovschi, V.; Vogt, L.; Vydrov, O.; Wang, T.; Watson, M. A.; Wenzel, J.; White, A.; Williams, C. F.; Yang, J.; Yeganeh, S.; Yost, S. R.; You, Z.-Q.; Zhang, I. Y.; Zhang, X.; Zhao, Y.; Brooks, B. R.; Chan, G. K. L.; Chipman, D. M.; Cramer, C. J.; Goddard, W. A.; Gordon, M. S.; Hehre, W. J.; Klamt, A.; Schaefer, H. F.; Schmidt, M. W.; Sherrill, C. D.; Truhlar, D. G.; Warshel, A.; Xu, X.; Aspuru-Guzik, A.; Baer, R.; Bell, A. T.; Besley, N. A.; Chai, J.-D.; Dreuw, A.; Dunietz, B. D.; Furlani, T. R.; Gwaltney, S. R.; Hsu, C.-P.; Jung, Y.; Kong, J.; Lambrecht, D. S.; Liang, W.; Ochsenfeld, C.; Rassolov, V. A.; Slipchenko, L. V.; Subotnik, J. E.; Van Voorhis, T.; Herbert, J. M.; Krylov, A. I.; Gill, P. M. W.; Head-Gordon, M., Advances in molecular quantum chemistry contained in the Q-Chem 4 program package. Mol. Phys. 2015, 113, 184-215. 
83. Ditchfield, R.; Hehre, W. J.; Pople, J. A., Self - Consistent Molecular - Orbital Methods. IX. An Extended Gaussian - Type Basis for Molecular - Orbital Studies of Organic Molecules. J. Chem. Phys. 1971, 54, 724-728.

84. Hariharan, P. C.; Pople, J. A., The influence of polarization functions on molecular orbital hydrogenation energies. Theo. Chim. Acta 1973, 28, 213-222.

85. Hehre, W. J.; Ditchfield, R.; Pople, J. A., Self-Consistent Molecular Orbital Methods. XII. Further Extensions of Gaussian-Type Basis Sets for Use in Molecular Orbital Studies of Organic Molecules. J. Chem. Phys. 1972, 56, 2257-2261.

86. Wolf, T. J. A.; Myhre, R. H.; Cryan, J. P.; Coriani, S.; Squibb, R. J.; Battistoni, A.; Berrah, N.; Bostedt, C.; Bucksbaum, P.; Coslovich, G.; Feifel, R.; Gaffney, K. J.; Grilj, J.; Martinez, T. J.; Miyabe, S.; Moeller, S. P.; Mucke, M.; Natan, A.; Obaid, R.; Osipov, T.; Plekan, O.; Wang, S.; Koch, H.; Guhr, M., Probing ultrafast pi pi*/n pi* internal conversion in organic chromophores via K-edge resonant absorption. Nat. Commun. 2017, 8.

87. Coe, J. D.; Martínez, T. J., Competitive Decay at Two- and Three-State Conical Intersections in Excited-State Intramolecular Proton Transfer. J. Amer. Chem. Soc. 2005, 127, 4560-4561.

88. Jacquemin, D.; Wathelet, V.; Perpète, E. A.; Adamo, C., Extensive TD-DFT Benchmark: Singlet-Excited States of Organic Molecules. J. Chem. Theo. Comp. 2009, 5, 2420-2435.

89. Risthaus, T.; Hansen, A.; Grimme, S., Excited States Using the Simplified Tamm-DancoffApproach for Range-Separated Hybrid Density Functionals: Development and Application. Phys. Chem. Chem. Phys. 2014, 28, 14408-14419.

90. Grimme, S.; Bannwarth, C., Ultra-fast computation of electronic spectra for large systems by tight-binding based simplified Tamm-Dancoff approximation (sTDA-xTB). J. Chem. Phys. 2016, $145,054103$.

91. Hellweg, A.; Grün, S. A.; Hättig, C., Benchmarking the performance of spin-component scaled CC2 in ground and electronically excited states. Phys. Chem. Chem. Phys. 2008, 10, 4119-4127.

92. Furche, F.; Ahlrichs, R.; Hättig, C.; Klopper, W.; Sierka, M.; Weigend, F., Turbomole. WIREs Comp. Mol. Sci. 2014, 4, 91-100.

93. Song, C.; Martínez, T. J., Reduced scaling CASPT2 using supporting subspaces and tensor hyper-contraction. J. Chem. Phys. 2018, 149, 044108.

94. Asturiol, D.; Lasorne, B.; Robb, M. A.; Blancafort, L., Photophysics of the $\pi, \pi^{*}$ and n, $\pi^{*}$ States of Thymine: MS-CASPT2 Minimum-Energy Paths and CASSCF on-the-Fly Dynamics. J. Phys. Chem. A 2009, 113, 10211-10218.

95. Segarra-Martí, J.; Francés-Monerris, A.; Roca-Sanjuán, D.; Merchán, M., Assessment of the Potential Energy Hypersurfaces in Thymine within Multiconfigurational Theory: CASSCF vs. CASPT2. Molecules 2016, 21. 


\section{Supporting Information:}

\section{Hole-hole Tamm-Dancoff-approximated density}

\section{functional theory: a highly efficient electronic}

structure method incorporating dynamic and

\section{static correlation}

Christoph Bannwarth, ${ }^{\dagger, \ddagger}$ Jimmy K. Yu, ${ }^{\dagger, \ddagger}, \mathbb{\uparrow}$ Edward G. Hohenstein, ${ }^{\dagger, \ddagger}$ and Todd J. Martínez $z^{* \dagger, \ddagger}$

$\dagger$ Department of Chemistry and The PULSE Institute, Stanford University, Stanford, CA 94305, United States of America.

$\ddagger S L A C$ National Accelerator Laboratory, 2575 Sand Hill Road, Menlo Park, CA 94025,

United States of America.

\Biophysics Program, Stanford University, Stanford, CA 94305, United States of America. 


\section{Contents}

List of Tables

1 Connection between spin-flip configuration interaction singles and $h h$-TDA S-4

2 Equivalence of FCI/STO-3G, $p p$-TDA-HF/STO-3G, and $h h$-TDA-HF/STO-

$3 \mathrm{G}$ for $\mathrm{H}_{2}$

3 Benchmarking vertical excitation energies at the Franck-Condon point with $h h$-TDA $\quad$ S-6

3.1 Intermolecular charge-transfer set . . . . . . . . . . . . . . . . S-7

3.2 Push-pull-type set . . . . . . . . . . . . . . . . . . . . . . . . S-9

3.3 Local excitation set . . . . . . . . . . . . . . . . . . . S-11

3.4 State-splittings . . . . . . . . . . . . . . . . . S . . . . . . . . . .

4 Basis set dependence of $h h$-TDA $\quad$ S-19

5 Comparison of $h h$-TDA and $p p$-TDA $\quad$ S-19

6 State positioning of the ethylene singlet $\pi \pi^{*}$ state by the $h h$-TDA and $p p$-TDA methods

7 Twisted-ethylene pyramidalization potential energy curve

References

S-26

\section{List of Tables}

S1 FCI, $p p$-TDA, and $h h$-TDA energies of $\mathrm{H}_{2}$ with STO-3G $\ldots \ldots . . . \quad$ S-6

S2 Mean field electronic structure methods considered . . . . . . . . . . . . . S-6

S3 Detailed results for global hybrids on the CT set . . . . . . . . . . . . S-7 
S4 Detailed results for range-separated functionals on the CT set . . . . . . . S-8

S5 Detailed results for global hybrids on the push-pull set . . . . . . . . . . S-10

S6 Detailed results for range-separated hybrids on the push-pull set . . . . . . . S-11

S7 Detailed results for global hybrids on the local excitation set . . . . . . . . S-13

S8 Detailed results for range-sparated hybrids on the local excitation set . . . . S-14

S9 Detailed results for global hybrids on the state splitting set . . . . . . . . S-16

S10 Detailed results for range-separated hybrids on the state splitting set . . . S-17

S11 Basis set dependence of excitation energies with $h h$-TDA- $\omega$ B97X . . . . . . . S-19

S12 Excitation energies computed with $h h$-TDA and $p p$-TDA . . . . . . . . . S-19

S13 Description of the ethylene singlet $\pi \pi^{*}$ state with $h h$-TDA and $p p$-TDA . . S-22 


\section{Connection between spin-flip configuration interac- tion singles and $h h$-TDA}

To see the connection between the spin-flip configuration interaction singles (SF-CIS) or spin-flip Tamm-Dancoff approximated particle-hole time-dependent density functional theory (SF-TDDFT) and $h h$-TDA, we start from a Hartree-Fock wavefunction as reference and look at the two-electron operator matrix element for two determinants generated from excitation and double annihilation. We look at the single particle-hole excitations first. In the following, $i, j, k, l$, refer to occupied, $a, b, c, d$ to virtual, and $p, q, r, s$ to either kind of orbitals. $|0\rangle$ refers to the occupation vector corresponding to the Hartree-Fock reference determinant. $[p q \mid r s]$ refers to a two-electron integral between spin-MOs and $(p q \mid r s)$ refers to a two-electron integral between spatial MOs both in chemists' notation.

$$
\begin{aligned}
\left\langle\Phi_{j}^{b}\left|\hat{g}_{p q r s}\right| \Phi_{i}^{a}\right\rangle & =\frac{1}{2} \sum_{p q, r s}[p q \mid r s]\left\langle 0\left|a_{j}^{\dagger} a_{b}\left\{a_{p}^{\dagger} a_{r}^{\dagger} a_{q} a_{s}\right\} a_{a}^{\dagger} a_{i}\right| 0\right\rangle \\
& =\frac{1}{2} \sum_{p q, r s}[p q \mid r s]\left(\delta_{p b} \delta_{q j} \delta_{r i} \delta_{s a}-\delta_{p i} \delta_{q j} \delta_{r b} \delta_{s a}-\delta_{p b} \delta_{q a} \delta_{r i} \delta_{s j}+\delta_{p i} \delta_{q a} \delta_{r b} \delta_{s j}\right) \\
& =\frac{1}{2}([b j \mid i a]-[i j \mid b a]-[b a \mid i j]+[i a \mid b j])=[i a \mid b j]-[i j \mid b a]
\end{aligned}
$$

In the spin-flip variant, $i, j \in\{\uparrow\}, a, b \equiv \bar{a}, \bar{b} \in\{\downarrow\}$, i.e., we get from Eq.1:

$$
\left\langle\Phi_{j}^{\bar{b}}\left|\hat{g}_{p q r s}\right| \Phi_{i}^{\bar{a}}\right\rangle=-(i j \mid \overline{b a})
$$

If we now look at the hole-hole case, we have

$$
\begin{aligned}
\left\langle\Phi_{j l}\left|\hat{g}_{p q r s}\right| \Phi_{i k}\right\rangle & =\frac{1}{2} \sum_{p q, r s}[p q \mid r s]\left\langle 0\left|\widehat{a_{j}^{\dagger} a_{l}^{\dagger}\left\{a_{p}^{\dagger} a_{r}^{\dagger} a_{q} a_{s}\right\}} a_{i} a_{k}\right| 0\right\rangle \\
& =\frac{1}{2} \sum_{p q, r s}[p q \mid r s]\left(\delta_{p k} \delta_{q l} \delta_{r i} \delta_{s j}-\delta_{p i} \delta_{q l} \delta_{r k} \delta_{s j}-\delta_{p k} \delta_{q j} \delta_{r i} \delta_{s l}+\delta_{p i} \delta_{q j} \delta_{r k} \delta_{s l}\right) \\
& =\frac{1}{2}([k l \mid i j]-[i l \mid k j]-[k j \mid i l]+[i j \mid k l])=[i j \mid k l]-[i l \mid k j] .
\end{aligned}
$$


In $h h$-TDA, we are annihilating two electrons of opposite spin, i.e., $i, j \in\{\uparrow\}, k, l \equiv \bar{k}, \bar{l} \in$ $\{\downarrow\}$. Hence, Eq.3 becomes

$$
\left\langle\Phi_{j \bar{l}}\left|\hat{g}_{p q r s}\right| \Phi_{i \bar{k}}\right\rangle=(i j \mid \overline{k l})
$$

Consequently, the matrix elements in $h h$-TDA-HF are of opposite sign, but otherwise equivalent to the ones in spin-flip configuration interaction singles (SF-CIS). The latter - and its density functional theory (DFT) counterpart - can be derived from linear-response theory and by applying the Tamm-Dancoff approximation. In that case, the non-vanishing two-electron integral in Eq.2 originates from the response of the Fock exchange term in the ground state ${ }^{\mathrm{S} 1}$ and, in commonly used density functional approximations (DFAs), occurs in its modified variant, i.e., with global scaling or range separation.

Due to the established connection between $h h$-TDA and SF-CIS on the one hand and the modification of the two-electron integral in collinear SF-TDDFT on the other, we choose to use accordingly modified two-electron integrals in DFT-based $h h$-TDA (and possibly $p p$ TDA).

We call this the linear response-type kernel, which enters the matrix elements with negative sign (see manuscript for a discussion). This kernel has been used in this functional assessment throughout:

$$
L_{i \bar{k}, j \bar{l}}=-a_{X}^{\mathrm{FR}}(i j \mid \overline{k l})-a_{X}^{\mathrm{LR}}(i j \mid \overline{k l})^{\mathrm{LR}}
$$




\section{Equivalence of FCI/STO-3G, $p p$-TDA-HF/STO-3G, and $h h$-TDA-HF/STO-3G for $\mathrm{H}_{2}$}

Table S1: Total energies (in Hartree) for $\mathrm{H}_{2}\left(R_{\mathrm{H}-\mathrm{H}}=0.776775 \AA\right)$ in an STO-3G basis with Hartree-Fock (HF), Full CI (FCI), $p p$-TDA-HF, and $h h$-TDA-HF.

\begin{tabular}{lrrrr}
\hline State & HF & FCI & $p p$-TDA & $h h$-TDA \\
\hline $1^{1} A_{1 g}$ & -1.1137477 & -1.1359353 & -1.1359353 & -1.1359353 \\
$1^{1} A_{2 u}$ & - & -0.2064462 & -0.2064462 & -0.2064462 \\
$2^{1} A_{1 g}$ & - & 0.4004024 & 0.4004024 & 0.4004024 \\
\hline
\end{tabular}

\section{Benchmarking vertical excitation energies at the Franck- Condon point with $h h$-TDA}

The performance of different density functionals for the calculation of vertical excitation energies in the linear-response time-dependent density functional theory framework is well known ${ }^{\mathrm{S} 2}$ and the role of Fock exchange is well understood. ${ }^{\mathrm{S}}$ This is different for $h h$-TDA, where the impact of global and range-separated Fock exchange on the lowest vertical excitation energies is not known. Hence we benchmark different literature-known density functional approximations (DFAs) along with Hartree-Fock (HF) in the calculation of vertical excitation energies for different excitation types (see below). The used functionals are given in Table S2.

Table S2: Mean field electronic structure methods considered here along with the amount of short- and long-range Fock exchange and range-sparation parameter $\omega$ (if applicable).

\begin{tabular}{lrrr}
\hline Method & $c_{\mathrm{HF}}\left(r_{12}=0\right)$ & $c_{\mathrm{HF}}\left(r_{12}=\infty\right)$ & $\omega$ \\
\hline Hartree-Fock (HF) & 1.00 & 1.00 & n.a. \\
B3LYP & 0.20 & 0.20 & n.a. \\
PBE0 & 0.25 & 0.25 & n.a. \\
BHLYP & 0.50 & 0.50 & n.a. \\
CAM-B3LYP & 0.19 & 0.65 & 0.33 \\
$\omega$ B97 & 0.00 & 1.00 & 0.40 \\
$\omega$ B97X & 0.157706 & 1.00 & 0.30 \\
$\omega$ B97X-D3 & 0.195728 & 1.00 & 0.25 \\
$\omega$ PBEh & 0.20 & 1.00 & 0.20 \\
\hline
\end{tabular}


We consider previously published benchmark sets ${ }^{\mathrm{S} 4-\mathrm{S} 6}$ that were re-grouped into intermolecular charge-transfer (CT), intramolecular CT (mostly push-pull systems), non-CT, as well as a local state-ordering set (mostly based on Ref. S6). The sets are discussed individually below. All $h h$-TDA calculations were carried out with a development version of the GPU-accelerated electronic structure code TeraChem. ${ }^{\mathrm{S} 7, \mathrm{~S} 8}$

\subsection{Intermolecular charge-transfer set}

For charge-transfer excitations, we use the non-covalent complexes in the set from Ref. S4. Here, the lowest vertical excitations correspond to intermolecular charge transfer (CT) excitations with the reference method, i.e., approximate coupled-cluster with singles and doubles (CC2). The considered molecules are given in Fig. S1. To identify the CT states, which are often not the lowest states with DFA-based $h h$-TDA, we checked for changes in the static dipole moments. Excitations with static dipole changes larger than 8 Debye were classified as CT states.

Table S3: Vertical excitations computed with $h$-TDA for different molecules with chargetransfer-type excitations (set taken from Ref. S4). Hartree Fock and different global hybrid functionals are considered and the spherical def2-SV $(\mathrm{P})^{\mathrm{S} 9, \mathrm{~S} 10}$ basis set is used throughout. The values are given in $\mathrm{eV}$ (dimensionless oscillator strength in parentheses). If the state of interest is not $S_{1}$, it is also denoted in parentheses.

\begin{tabular}{|c|c|c|c|c|c|}
\hline system & B3LYP & PBE0 & BHLYP & $\mathrm{HF}$ & ref. ${ }^{a}$ \\
\hline Chloranil-diphenylene & $3.14\left(0.034, S_{3}\right)$ & $3.20\left(0.035, S_{2}\right)$ & $3.26(0.057)$ & $1.90(0.140)$ & 2.81 \\
\hline Chloranil-hexamethylbenzene & $4.15\left(0.028, S_{5}\right)$ & $2.72\left(0.000, S_{5}\right)$ & $3.21(0.000)$ & $2.15(0.000)$ & 2.87 \\
\hline TCNE-benzene & $5.13\left(0.000, S_{4}\right)$ & $5.04\left(0.000, S_{3}\right)$ & $4.34(0.000)$ & $2.86(0.000)$ & 3.78 \\
\hline TCNE-diphenylene & $-b$ & $3.12(0.008)$ & $3.00(0.014)$ & $1.22(0.045)$ & 2.28 \\
\hline TCNE-hexamethylbenzene & $3.73\left(0.001, S_{2}\right)$ & $3.61(0.001)$ & $2.94(0.000)$ & $1.59(0.000)$ & 2.36 \\
\hline TCNE-o-xylene & $4.57\left(0.012, S_{2}\right)$ & $4.46\left(0.011, S_{2}\right)$ & $3.77(0.011)$ & $2.31(0.017)$ & 3.17 \\
\hline MD: & 1.15 & 1.03 & 0.54 & -0.87 & - \\
\hline MAD: & 1.15 & 1.03 & 0.54 & 0.87 & - \\
\hline RMSD: & 1.22 & 1.08 & 0.55 & 0.88 & - \\
\hline SD: & 0.46 & 0.36 & 0.13 & 0.12 & - \\
\hline MAX: & 1.40 & 1.29 & 0.72 & 1.06 & - \\
\hline
\end{tabular}


<smiles>O=C1C(Cl)=C(Cl)C(=O)C(Cl)=C1Cl</smiles>

Chloranil-diphenylene<smiles>N#CC(C#N)=C(C#N)C#N</smiles><smiles>Cc1c(C)c(C)c(C)c(C)c1C</smiles>

TCNE-hexamethylbenzene<smiles>Cc1c(C)c(C)c(C)c(C)c1C</smiles>

Chloranil-hexamethylbenzene<smiles>CC(C#N)=C(C#N)C#N</smiles><smiles>c1ccc2c(c1)-c1ccccc1-2</smiles>

TCNE-diphenylene<smiles>CC(C#N)=C(C#N)C#N</smiles><smiles>Cc1ccccc1C</smiles>

TCNE-o-Xylene

Figure S1: Molecules considered in the intermolecular charge-transfer set. TCNE = tetracyanoethylene.

Table S4: Vertical excitations computed with $h h$-TDA for different molecules with chargetransfer-type excitations (set taken from Ref. S4). Different range-separated hybrid functionals are considered and the spherical def2-SV $(\mathrm{P})^{\mathrm{S} 9, \mathrm{~S} 10}$ basis set is used throughout. The values are given in $\mathrm{eV}$ (dimensionless oscillator strength in parentheses). If the state of interest is not $S_{1}$, it is also denoted in parentheses.

\begin{tabular}{|c|c|c|c|c|c|c|}
\hline system & CAM-B3LYP & $\omega \mathrm{PBEh}$ & $\omega \mathrm{B} 97$ & $\omega \mathrm{B} 97 \mathrm{X}$ & $\omega \mathrm{B} 97 \mathrm{X}-\mathrm{D} 3$ & ref. $^{a}$ \\
\hline Chloranil-diphenylene & $3.49\left(0.054, S_{4}\right)$ & $3.39\left(0.053, S_{4}\right)$ & $3.05\left(0.076, S_{2}\right)$ & $3.09\left(0.069, S_{2}\right)$ & $3.17\left(0.063, S_{3}\right)$ & 2.81 \\
\hline Chloranil-hexamethylbenzene & $3.56\left(0.039, S_{4}\right)$ & $3.47\left(0.038, S_{4}\right)$ & $3.04\left(0.000, S_{2}\right)$ & $3.05\left(0.000, S_{2}\right)$ & $3.28\left(0.045, S_{4}\right)$ & 2.87 \\
\hline TCNE-benzene & $4.63\left(0.000, S_{2}\right)$ & $4.60\left(0.000, S_{2}\right)$ & $4.25(0.000)$ & $4.30(0.000)$ & $4.37\left(0.000, S_{2}\right)$ & 3.78 \\
\hline TCNE-diphenylene & $3.27(0.013)$ & $3.20(0.014)$ & $2.71(0.020)$ & $2.80(0.018)$ & $2.91(0.017)$ & 2.28 \\
\hline TCNE-hexamethylbenzene & $3.17(0.001)$ & $3.15(0.001)$ & $2.78(0.000)$ & $2.84(0.000)$ & $2.92(0.000)$ & 2.36 \\
\hline TCNE-o-xylene & $-\dot{b}$ & $3.99(0.012)$ & $3.62(0.014)$ & $3.68(0.013)$ & $3.76(0.013)$ & 3.17 \\
\hline MD: & 0.80 & 0.76 & 0.36 & 0.41 & 0.52 & - \\
\hline MAD: & 0.80 & 0.76 & 0.36 & 0.41 & 0.52 & - \\
\hline RMSD: & 0.81 & 0.76 & 0.38 & 0.43 & 0.53 & - \\
\hline SD: & 0.13 & 0.14 & 0.12 & 0.15 & 0.11 & - \\
\hline MAX: & 0.99 & 0.92 & 0.47 & 0.52 & 0.63 & - \\
\hline
\end{tabular}


(a)

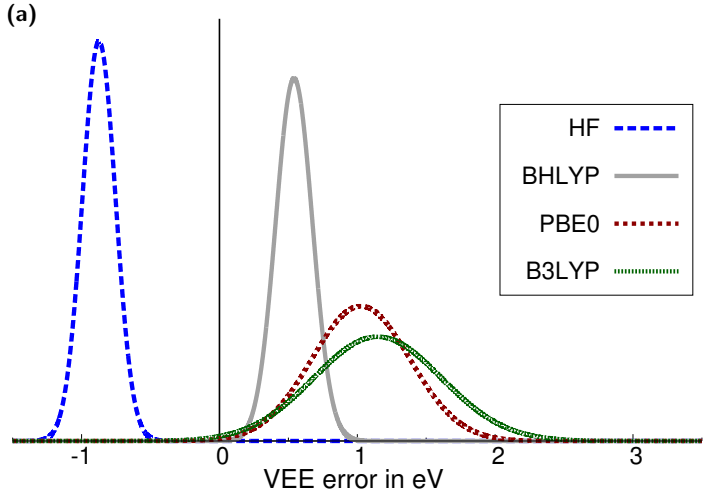

(b)

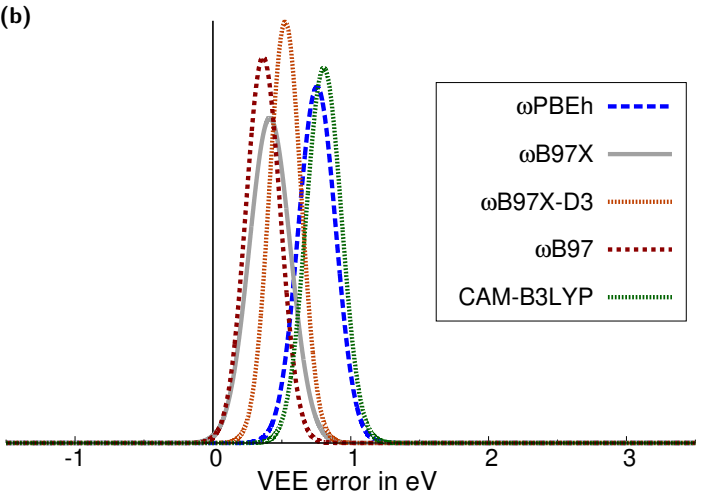

Figure S2: Gaussian error distribution functions for $h h$-TDA with different standard density functionals in the calculation of intermolecular charge-transfer (CT) excitation energies. The spherical def2-SV $(\mathrm{P})^{\mathrm{S} 9, \mathrm{~S} 10}$ basis set is used throughout. The centers of the Gaussians correspond to the mean deviation (MD) and the width of the Gaussian corresponds to the standard deviation (SD) for the vertical excitation energies on the CT set (see Tables S3 and S4 for details).

\subsection{Push-pull-type set}

For monomolecular systems with dipole-allowed excitations, that have some intramolecular charge-transfer character, we use the monomolecular systems from the set in Ref. S4 and added a few large molecules ( $>20$ atoms) from the set presented in Ref. S5. From the latter set, such systems were chosen if the TDA-PBE0/def2-SV(P) were lower than the reference value, i.e., we take this underestimation as a measure for $\mathrm{CT}$ character in that excitation. These dipole-allowed, intramolecular CT excitations are commonly found in so-called pushpull systems, hence, we refer to this set as the "push-pull-type" excitations in the following. The considered molecules are given in Fig. S1. 
<smiles>CN(C)c1ccc2c(C(F)(F)F)cc(=O)oc2c1</smiles>

Coumarin-152

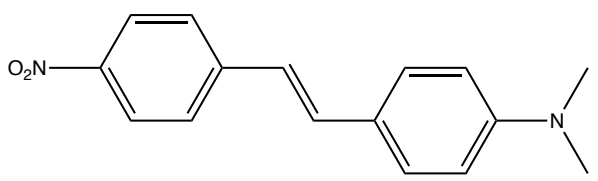

DANS

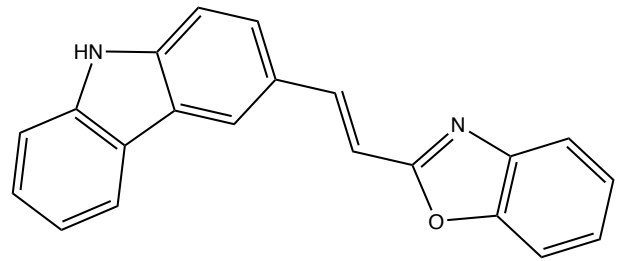

S0904
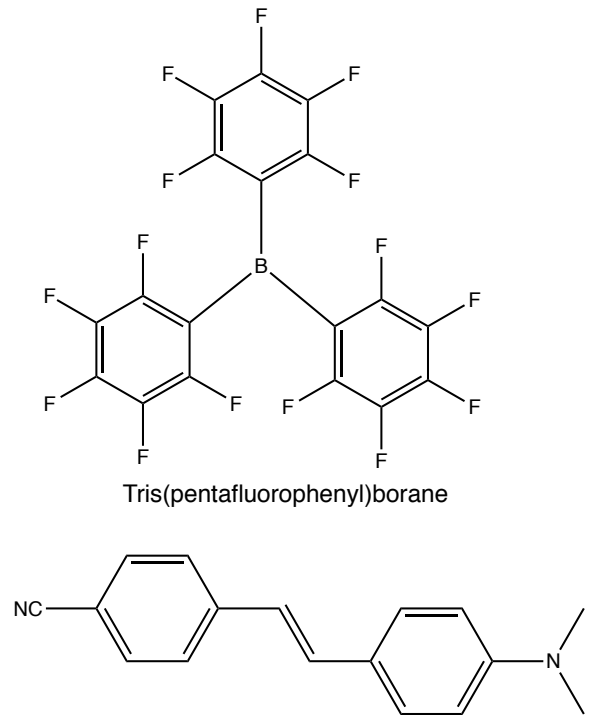

DCS

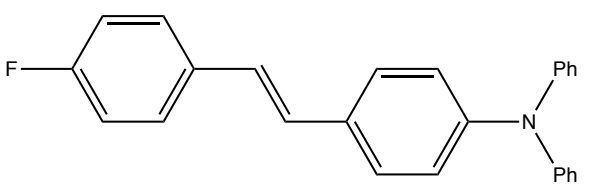

S2127

Figure S3: Molecules considered in the push-pull-type excitation set. DANS: 4Dimethylamino-4'-nitrostilbene, DCS: 4-Dimethylamino-4'-cyanostilbene, S0904: (E)-1-(2Carbazyl)-2-(2-benzoxazyl)-ethylene, S2127: 4-Diphenylamino-4'-fluorostilbene.

Table S5: Vertical excitations computed with hh-TDA for push-pull-type molecules with partial intramolecular charge-transfer-type excitations (systems taken from Ref. S4 and S5). Hartree Fock and different global hybrid functionals are considered and the spherical def2$\mathrm{SV}(\mathrm{P})^{\mathrm{S} 9, \mathrm{~S} 10}$ basis set is used throughout. The values are given in $\mathrm{eV}$ (dimensionless oscillator strength in parentheses). If not denoted otherwise, the reference values are taken from Ref. S4.

\begin{tabular}{lrrrrr}
\hline system & B3LYP & PBE0 & BHLYP & HF & ref. $^{a}$ \\
\hline $\mathrm{B}\left(\mathrm{C}_{6} \mathrm{~F}_{6}\right)_{3}$ & $4.02(0.109)$ & $4.07(0.108)$ & $4.44(0.107)$ & $4.82(0.093)$ & $4.10^{b}$ \\
Coumarin-152 & $3.52(1.167)$ & $3.64(1.236)$ & $4.10(1.423)$ & $4.75(1.611)$ & 3.69 \\
DANS & $2.85(2.093)$ & $2.97(2.231)$ & $3.46(2.719)$ & $3.89(2.930)$ & 3.42 \\
DCS & $2.93(2.258)$ & $3.06(2.395)$ & $3.58(2.914)$ & $4.16(3.250)$ & 3.56 \\
S0904 & $3.24(1.491)$ & $3.39(1.666)$ & $3.93(2.535)$ & $4.26(3.407)$ & $3.81^{b}$ \\
S2127 & $3.12(1.647)$ & $3.27(1.852)$ & $3.94(2.698)$ & $4.50(2.683)$ & $3.66^{b}$ \\
\hline MD: & -0.43 & -0.31 & 0.20 & 0.69 & - \\
MAD: & 0.43 & 0.31 & 0.20 & 0.69 & - \\
RMSD: & 0.48 & 0.36 & 0.25 & 0.72 & - \\
SD: & 0.23 & 0.21 & 0.16 & 0.23 & - \\
MAX: & 0.63 & 0.50 & 0.41 & 1.06 & - \\
\hline
\end{tabular}

${ }^{a}$ SCS-CC2/def2-TZVP(-f) from Ref. S4. ${ }^{b}$ SCS-CC2/aug-cc-pVDZ from Ref. S5. DANS:

4-Dimethylamino-4'-nitrostilbene, DCS: 4-Dimethylamino-4'-cyanostilbene, S0904:

(E)-1-(2-Carbazyl)-2-(2-benzoxazyl)-ethylene, $\$ 2$ 12 6 : 4-Diphenylamino-4'-fluorostilbene. 
(a)

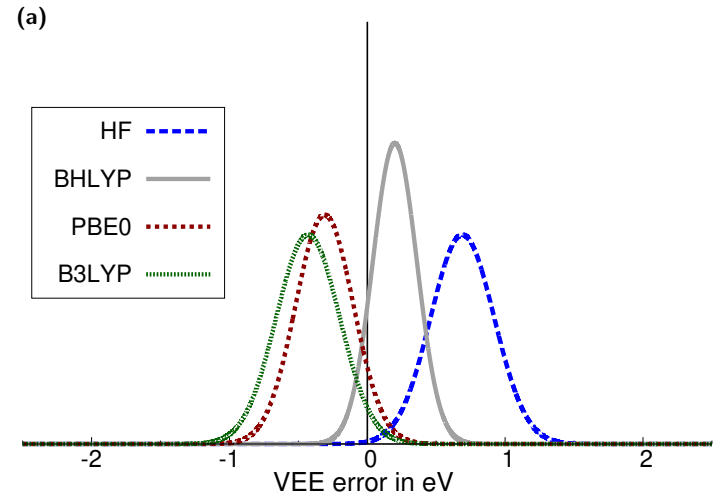

(b)

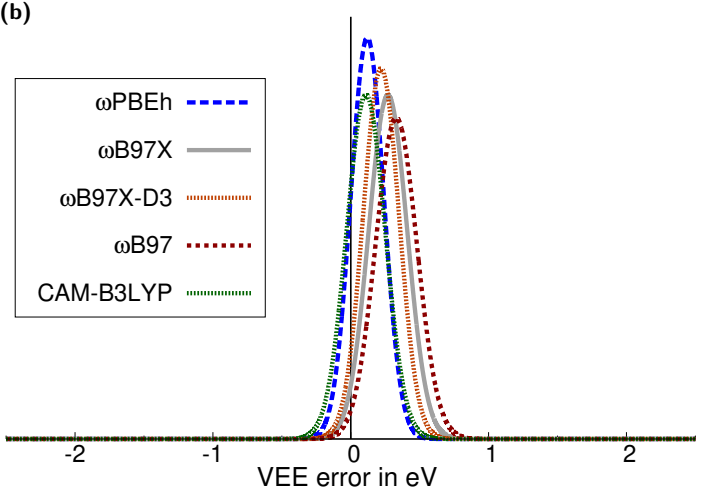

Figure S4: Gaussian error distribution functions for $h h$-TDA with different standard density functionals in the calculation of vertical push-pull-type excitation energies. The spherical def2-SV $(\mathrm{P})^{\mathrm{S} 9, \mathrm{~S} 10}$ basis set is used throughout. The center of the Gaussian corresponds to the mean deviation (MD) and the width of the Gaussian corresponds to the standard deviation (SD) for the vertical excitation energies on the push-pull set (see Tables S5 and S6 for details).

Table S6: Vertical excitations computed with $h h$-TDA for push-pull-type molecules with partial intramolecular charge-transfer-type excitations (set taken from Ref. S4 and S5). Different range-separated hybrid functionals are considered and the spherical def2-SV $(\mathrm{P})^{\mathrm{S} 9, \mathrm{~S} 10}$ basis set is used throughout. The values are given in $\mathrm{eV}$ (dimensionless oscillator strength in parentheses). If not denoted otherwise, the reference values are taken from Ref. S4.

\begin{tabular}{|c|c|c|c|c|c|c|}
\hline system & CAM-B3LYP & $\omega \mathrm{PBEh}$ & $\omega \mathrm{B} 97$ & $\omega \mathrm{B} 97 \mathrm{X}$ & $\omega \mathrm{B} 97 \mathrm{X}-\mathrm{D} 3$ & ref. $^{a}$ \\
\hline $\mathrm{B}\left(\mathrm{C}_{6} \mathrm{~F}_{6}\right)_{3}$ & $4.29(0.103)$ & $4.22(0.096)$ & $4.42(0.094)$ & $4.37(0.094)$ & $4.32(0.095)$ & $4.10^{b}$ \\
\hline Coumarin-152 & $3.98(1.447)$ & 3.97 (1.468) & $4.26(1.569)$ & $4.16(1.529)$ & $4.08(1.503)$ & 3.69 \\
\hline DANS & $3.43(2.784)$ & $3.47(2.815)$ & $3.67(2.954)$ & $3.60(2.877)$ & $3.55(2.843)$ & 3.42 \\
\hline DCS & 3.49 (2.979) & $3.53(3.018)$ & $3.73(3.235)$ & $3.67(3.137)$ & $3.62(3.082)$ & 3.56 \\
\hline S0904 & $3.85(2.583)$ & $3.88(2.768)$ & $4.04(3.116)$ & $3.99(3.011)$ & $3.95(2.928)$ & $3.81^{b}$ \\
\hline $\mathrm{S} 2127$ & $3.85(2.859)$ & 3.91 (3.039) & $4.11(3.135)$ & $4.07(3.090)$ & $4.02(3.077)$ & $3.66^{b}$ \\
\hline MD: & 0.11 & 0.12 & 0.33 & 0.27 & 0.22 & - \\
\hline MAD: & 0.13 & 0.13 & 0.33 & 0.27 & 0.22 & - \\
\hline RMSD: & 0.57 & 0.17 & 0.36 & 0.30 & 0.25 & - \\
\hline SD: & 0.14 & 0.12 & 0.15 & 0.14 & 0.13 & - \\
\hline MAX: & 0.29 & 0.28 & 0.57 & 0.47 & 0.39 & - \\
\hline
\end{tabular}

${ }^{a}$ SCS-CC2/def2-TZVP(-f) from Ref. S4. ${ }^{b}$ SCS-CC2/aug-cc-pVDZ from Ref. S5. DANS:

4-Dimethylamino-4'-nitrostilbene, DCS: 4-Dimethylamino-4'-cyanostilbene, S0904:

(E)-1-(2-Carbazyl)-2-(2-benzoxazyl)-ethylene, S2127: 4-Diphenylamino-4'-fluorostilbene.

\subsection{Local excitation set}




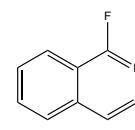

fluorisocholine

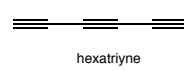

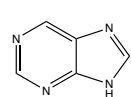

purine
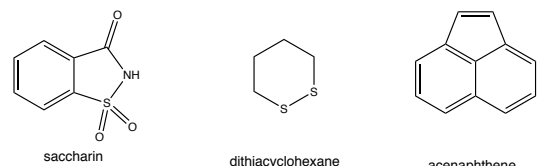

dithiacyclohexane

acenaphthene

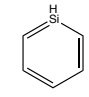

silabenzene
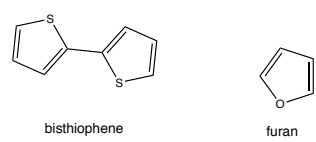

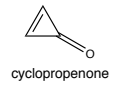

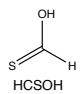

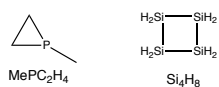

$\underset{\mathrm{P}_{2} \mathrm{P}}{\mathrm{H}_{4}}-\mathrm{PH}_{2}$

$\overbrace{\mathrm{CF}_{3} \mathrm{COOH}}^{\mathrm{OH}}$
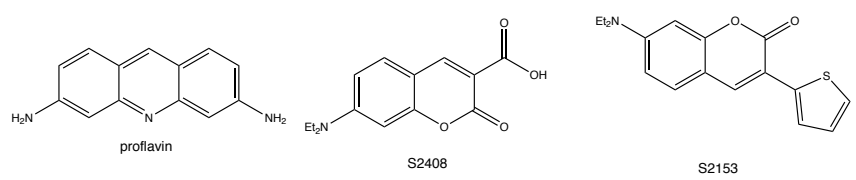

S2153
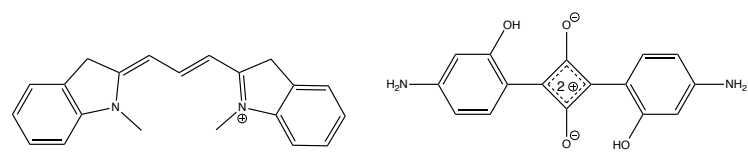

Figure S5: Molecules considered in the local excitation set. S2408: 7-diethylamino-2oxo-2H-chromene-3-carboxylic acid. S0491: 1-methyl-indole based Cy3 dye. S2153: 7diethylamino-3-thiophen-2-yl-chromen-2-one S2084: 1,3-bis[4-amino-2-hydroxyphenyl]-2,4dioxy-cyclobutene
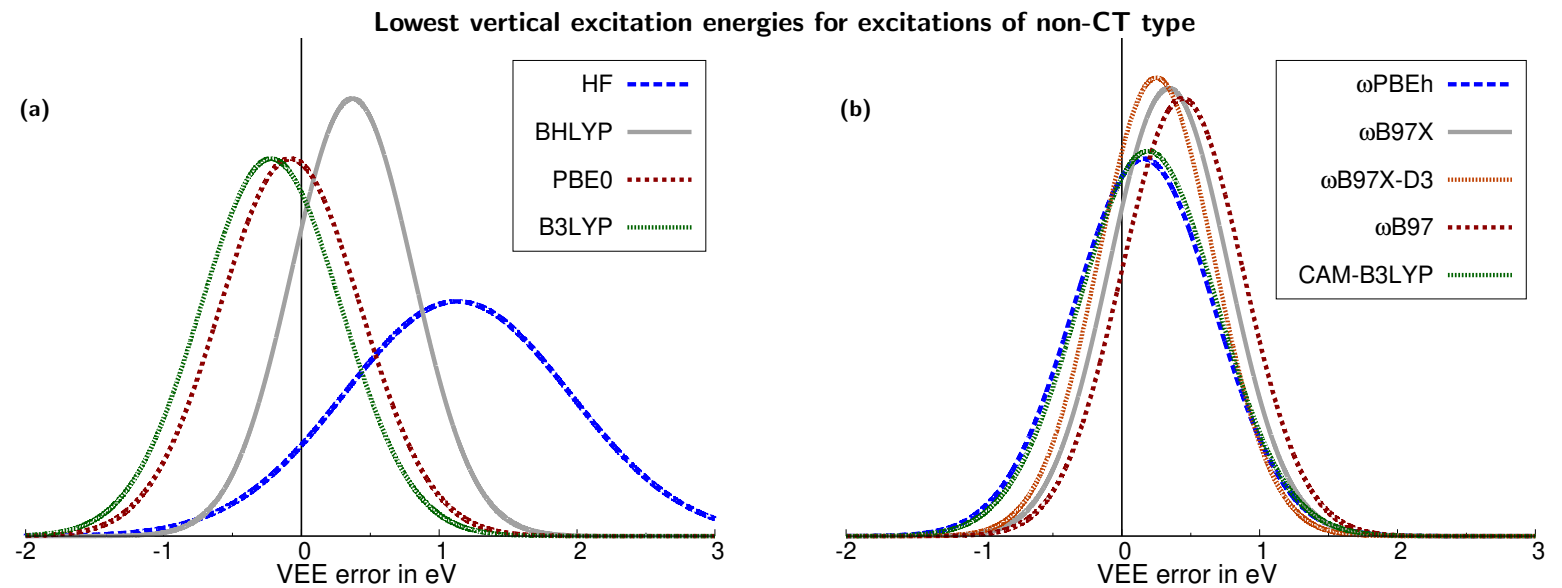

Figure S6: Gaussian error distribution functions for $h h$-TDA with different standard density functionals in the calculation of lowest vertical excitation energies with no (or little) chargetransfer character. The spherical def2-SV $(\mathrm{P})^{\mathrm{S} 9, \mathrm{~S} 10}$ basis set is used throughout. The center of the Gaussian corresponds to the mean deviation (MD) and the width of the Gaussian corresponds to the standard deviation (SD) for the vertical excitation energies on the nonCT set (see Tables S7 and S8 for details). 
Table S7: Lowest vertical excitations computed with $h h$-TDA for different molecules with predominantly local lowest vertical excitations (unless noted otherwise, systems are taken from Ref. S5). Hartree Fock and different global hybrid functionals are considered and the spherical def2-SV $(\mathrm{P})^{\mathrm{S} 9, \mathrm{~S} 10}$ basis set is used throughout. The values are given in $\mathrm{eV}$ (dimensionless oscillator strength in parentheses).

\begin{tabular}{|c|c|c|c|c|c|}
\hline system & B3LYP & PBE0 & BHLYP & $\mathrm{HF}$ & ref. $^{a}$ \\
\hline Acenaphthylene & $3.02(0.071)$ & $3.13(0.066)$ & $3.54(0.040)$ & $4.26(0.021)$ & $3.65(0.000)$ \\
\hline Bisthiophen & $3.59(1.033)$ & $3.76(1.091)$ & $4.39(1.329)$ & $5.48(1.738)$ & $4.48(0.395)$ \\
\hline $\mathrm{CF}_{3} \mathrm{COOH}$ & $6.21(0.040)$ & $6.39(0.039)$ & $6.34(0.007)$ & $6.72(0.006)$ & $5.95(0.000)$ \\
\hline Cyclopropenon & $4.77(0.008)$ & $4.73(0.008)$ & $4.61(0.006)$ & $4.47(0.004)$ & $4.42(0.000)$ \\
\hline Dithiacyclohexan & $4.88(0.077)$ & $5.04(0.077)$ & $5.58(0.105)$ & $5.86(0.045)$ & $4.52(0.009)$ \\
\hline Ethylene & $6.31(0.563)$ & $6.60(0.578)$ & $7.94(0.622)$ & $10.69(0.714)^{c}$ & $7.80(0.410)^{b}$ \\
\hline Fluorisochinolin & $4.16(0.432)$ & $4.32(0.444)$ & $4.97(0.486)$ & $5.98(0.504)$ & $4.50(0.028)$ \\
\hline Furan & $5.65(0.208)$ & $5.69(0.367)$ & $6.43(0.387)$ & $8.11(0.466)$ & $6.32(0.159)^{b}$ \\
\hline $\mathrm{HCSOH}$ & $4.22(0.001)$ & $4.21(0.001)$ & $3.77(0.000)$ & $3.43(0.000)$ & $3.57(0.000)$ \\
\hline Hexatriyne & $4.13(0.000)$ & $4.22(0.000)$ & $4.38(0.000)$ & $4.73(0.000)$ & $4.85(0.000)$ \\
\hline $\mathrm{MePC}_{2} \mathrm{H}_{4}$ & $7.05(0.047)$ & $7.24(0.054)$ & $8.13(0.051)$ & $8.92(0.224)$ & $6.53(0.040)$ \\
\hline $\mathrm{P}_{2} \mathrm{H}_{4}$ & $6.21(0.027)$ & $6.38(0.028)$ & $6.94(0.763)$ & $7.48(0.021)$ & $6.25(0.050)$ \\
\hline Proflavin & $3.01(0.177)$ & $3.11(0.175)$ & $3.56(0.176)$ & $4.23(0.151)$ & $3.54(0.234)$ \\
\hline Purine & $4.52(0.002)$ & $4.63(0.002)$ & $5.39(0.002)$ & $6.77(0.004)$ & $4.69(0.003)$ \\
\hline S0491 & $2.61(1.964)$ & $2.73(2.088)$ & $3.03(2.350)$ & $2.82(2.191)$ & $2.70(1.548)$ \\
\hline S2084 & $2.22(1.902)$ & $2.31(2.022)$ & $2.69(2.470)$ & $3.40(2.705)$ & $2.37(1.423)$ \\
\hline S2153 & $3.04(1.671)$ & $3.17(1.783)$ & $3.61(2.162)$ & $4.18(2.606)$ & $3.48(1.004)$ \\
\hline S2408 & $3.48(1.464)$ & $3.61(1.543)$ & $4.12(1.798)$ & $4.98(2.151)$ & $3.64(0.838)$ \\
\hline Saccharin & $4.56(0.002)$ & $4.69(0.002)$ & $5.37(0.002)$ & $6.46(0.003)$ & $4.91(0.004)$ \\
\hline $\mathrm{Si}_{4} \mathrm{H}_{8}$ & $5.10(0.000)$ & $5.14(0.000)$ & $5.51(0.000)$ & $6.13(0.000)$ & $5.22(0.000)$ \\
\hline Silabenzene & $4.19(0.559)$ & $4.82(0.550)$ & $5.00(0.653)$ & $6.27(0.793)$ & $4.23(0.059)$ \\
\hline MD: & -0.22 & -0.08 & 0.37 & 1.13 & - \\
\hline MAD: & 0.43 & 0.40 & 0.43 & 1.15 & - \\
\hline RMSD: & 0.54 & 0.50 & 0.57 & 1.39 & - \\
\hline SD: & 0.51 & 0.51 & 0.44 & 0.82 & - \\
\hline MAX: & 1.49 & 1.20 & 1.60 & 2.89 & - \\
\hline
\end{tabular}

${ }^{a}$ Structures and reference values (SCS-CC2/aug-cc-pVXZ $(\mathrm{X}=\mathrm{D}, \mathrm{T})$ ) from Ref. S5. ${ }^{b}$ Best estimate energy value from Ref. S6 (CCSD value for oscillator strength). ${ }^{c}$ Second lowest excited state. 
Table S8: Lowest vertical excitations computed with $h$-TDA for different molecules with predominantly local lowest vertical excitations (unless noted otherwise, systems are taken from Ref. S5). Different range-sparated hybrid functionals are considered and the spherical def2-SV $(\mathrm{P})^{\mathrm{S} 9, \mathrm{~S} 10}$ basis set is used throughout. The values are given in $\mathrm{eV}$ (dimensionless oscillator strength in parentheses).

\begin{tabular}{|c|c|c|c|c|c|c|}
\hline system & CAM-B3LYP & $\omega \mathrm{PBEh}$ & $\omega \mathrm{B} 97$ & $\omega \mathrm{B} 97 \mathrm{X}$ & $\omega \mathrm{B} 97 \mathrm{X}-\mathrm{D} 3$ & ref. $^{a}$ \\
\hline Acenaphthylene & $3.45(0.067)$ & $3.45(0.066)$ & $3.78(0.076)$ & $3.68(0.068)$ & $3.59(0.065)$ & $3.65(0.000)$ \\
\hline Bisthiophen & $4.22(1.326)$ & $4.27(1.355)$ & $4.69(1.564)$ & $4.57(1.491)$ & $4.45(1.435)$ & $4.48(0.395)$ \\
\hline $\mathrm{CF}_{3} \mathrm{COOH}$ & $6.17(0.009)$ & $6.15(0.010)$ & $6.29(0.010)$ & $6.23(0.009)$ & $6.20(0.009)$ & $5.95(0.000)$ \\
\hline Cyclopropenon & $4.50(0.006)$ & $4.53(0.007)$ & $4.25(0.006)$ & $4.34(0.006)$ & $4.42(0.006)$ & $4.42(0.000)$ \\
\hline Dithiacyclohexan & $5.39(0.111)$ & $5.18(0.094)$ & $5.59(0.079)$ & $5.53(0.092)$ & $5.38(0.092)$ & $4.52(0.009)$ \\
\hline Ethylene & $7.03(0.602)$ & $6.85(0.597)$ & $7.51(0.643)$ & $7.32(0.623)$ & $7.13(0.611)$ & $7.80(0.410)^{b}$ \\
\hline Fluorisochinolin & $4.70(0.474)$ & $4.65(0.467)$ & $5.10(0.504)$ & $4.97(0.489)$ & $4.84(0.480)$ & $4.50(0.028)$ \\
\hline Furan & $5.98(0.381)$ & $5.90(0.381)$ & $6.37(0.412)$ & $6.20(0.397)$ & $6.07(0.389)$ & $6.32(0.159)^{b}$ \\
\hline $\mathrm{HCSOH}$ & $4.09(0.001)$ & $4.21(0.001)$ & $4.25(0.001)$ & $4.13(0.001)$ & $4.12(0.001)$ & $3.57(0.000)$ \\
\hline Hexatriyne & $4.39(0.000)$ & $4.41(0.000)$ & $4.65(0.000)$ & $4.54(0.000)$ & $4.47(0.000)$ & $4.85(0.000)$ \\
\hline $\mathrm{MePC}_{2} \mathrm{H}_{4}$ & $8.08(0.059)$ & $8.06(0.106)$ & $7.87(0.231)$ & $7.65(0.219)$ & $7.47(0.209)$ & $6.53(0.040)$ \\
\hline $\mathrm{P}_{2} \mathrm{H}_{4}$ & $6.76(0.813)$ & $6.70(0.842)$ & $7.07(0.014)$ & $7.01(0.017)$ & $6.89(0.800)$ & $6.25(0.050)$ \\
\hline Proflavin & $3.40(0.163)$ & $3.35(0.138)$ & $3.65(0.140)$ & $3.55(0.136)$ & $3.47(0.137)$ & $3.54(0.234)$ \\
\hline Purine & $5.02(0.002)$ & $4.87(0.002)$ & $5.36(0.003)$ & $5.20(0.003)$ & $5.08(0.002)$ & $4.69(0.003)$ \\
\hline S0491 & $3.09(2.401)$ & $3.13(2.451)$ & $3.19(2.418)$ & $3.16(2.415)$ & $3.15(2.425)$ & $2.70(1.548)$ \\
\hline S2084 & $2.70(2.479)$ & $2.82(2.693)$ & $3.07(2.853)$ & $2.98(2.829)$ & $2.92(2.790)$ & $2.37(1.423)$ \\
\hline S2153 & $3.53(2.169)$ & $3.56(2.248)$ & $3.82(2.509)$ & $3.74(2.418)$ & $3.66(2.347)$ & $3.48(1.004)$ \\
\hline S2408 & $4.02(1.822)$ & $4.02(1.864)$ & $4.38(2.029)$ & $4.25(1.974)$ & $4.16(1.928)$ & $3.64(0.838)$ \\
\hline Saccharin & $4.86(0.002)$ & $4.70(0.002)$ & $4.94(0.002)$ & $4.88(0.002)$ & $4.83(0.002)$ & $4.91(0.004)$ \\
\hline $\mathrm{Si}_{4} \mathrm{H}_{8}$ & $5.53(0.000)$ & $5.46(0.000)$ & $5.81(0.000)$ & $5.70(0.000)$ & $5.61(0.000)$ & $5.22(0.000)$ \\
\hline Silabenzene & $4.76(0.647)$ & $4.71(0.644)$ & $5.22(0.729)$ & $5.06(0.697)$ & $4.92(0.675)$ & $4.23(0.059)$ \\
\hline MD: & 0.19 & 0.16 & 0.44 & 0.34 & 0.25 & - \\
\hline MAD: & 0.40 & 0.41 & 0.50 & 0.43 & 0.40 & - \\
\hline RMSD: & 0.52 & 0.52 & 0.61 & 0.54 & 0.48 & - \\
\hline SD: & 0.50 & 0.51 & 0.44 & 0.43 & 0.42 & - \\
\hline MAX: & 1.55 & 1.53 & 1.34 & 1.12 & 0.94 & - \\
\hline
\end{tabular}

\subsection{State-splittings}

Except for aspirin (taken from Ref. S5), the systems considered in this set are taken from Thiel's 2008 benchmark set. ${ }^{\text {S6 }}$ From these, problematic systems and states were discarded: aromatic systems, where the population of the LUMO in the $(N+2)$ reference is not clear (i.e., benzene, naphthalene, pyrrole, s-triazine) or if the excited states cannot predominantly be described by a occupied orbital-to-LUMO transition, according to TD-BHLYP/TZVP calculations (i.e., if $\left.\left(X^{2}-Y^{2}\right)_{i \rightarrow \text { LUMO }}<0.50\right)$. Furthermore, butadiene, hexatriene, and octatetraene are not considered here, because we are interested in the state splitting between singly excited states. It is noted here, that $h h$-TDA can describe the doubly excited states, 
but that these are blue-shifted in the linear-response-type response kernel formulation with any $h h$-TDA functional combination (i.e., the state ordering for hexatriene and octatetraene is incorrect). Ethylene and furan (lowest state) in Thiel's set have been moved to the lowest vertical local excitation set (see above).

In the state-splitting set below, two states are included for each molecule. The only exception is pyrimidine where four states are considered. The states were selected by means of TDBHLYP/TZVP calculations and identified to be dominated by excitations to the $\pi^{*}$ LUMO. The latter calculations were done in Turbomole ${ }^{\mathrm{S} 11-\mathrm{S} 13}$ and also used to confirm the state characters. 
Table S9: Vertical excitation energies computed with $h h$-TDA for different states on small molecule. Unless noted otherwise, systems are taken from Ref. S6. Hartree Fock and different global hybrid functionals are considered and the spherical def2-SV $(\mathrm{P})^{\mathrm{S} 9, \mathrm{~S} 10}$ basis set is used throughout. The values are given in $\mathrm{eV}$ (dimensionless oscillator strength in parentheses).

\begin{tabular}{|c|c|c|c|c|c|c|c|}
\hline \multirow{2}{*}{$\frac{\text { system }}{\text { Acetamide }}$} & \multicolumn{2}{|c|}{ state } & \multirow{2}{*}{$\begin{array}{r}\text { B3LYP } \\
-^{c}\end{array}$} & \multirow{2}{*}{$\begin{array}{r}\text { PBE0 } \\
-^{c}\end{array}$} & \multirow{2}{*}{$\frac{\text { BHLYP }}{-^{c}}$} & \multirow{2}{*}{$\begin{array}{r}\mathrm{HF} \\
8.11(0.018)\end{array}$} & \multirow{2}{*}{$\begin{array}{r}\text { ref. }^{a} \\
5.80(0.001)\end{array}$} \\
\hline & $1^{1} A^{\prime \prime}$ & $n \rightarrow \pi^{*}$ & & & & & \\
\hline & $2^{1} A^{\prime}$ & $\pi \rightarrow \pi^{*}$ & $-^{c}$ & $-{ }^{c}$ & $-^{c}$ & $10.10(0.370)$ & $7.27(0.223)$ \\
\hline \multirow[t]{2}{*}{ Acetone } & $1^{1} A_{2}$ & $n \rightarrow \pi^{*}$ & $-^{c}$ & $-^{c}$ & $-^{c}$ & $6.67(0.000)$ & $4.40(0.000)$ \\
\hline & $2^{1} A_{1}$ & $\pi \rightarrow \pi^{*}$ & $-^{c}$ & $-^{c}$ & $-^{c}$ & $11.51(1.093)$ & $9.40(0.256)$ \\
\hline \multirow[t]{2}{*}{ Adenine } & $1^{1} A^{\prime \prime}$ & $n \rightarrow \pi^{*}$ & $5.25(0.000)$ & $5.40(0.000)$ & $6.24(0.000)$ & $7.87(0.001)$ & $5.12(0.001)$ \\
\hline & $3^{1} A^{\prime}$ & $\pi \rightarrow \pi^{*}$ & $4.75(0.811)$ & $4.94(0.839)$ & $5.71(0.941)$ & $7.15(1.123)$ & $5.25(0.297)$ \\
\hline \multirow[t]{2}{*}{ Aspirin } & $2^{1} A$ & $\pi \rightarrow \pi^{*}$ & $4.84(0.318)$ & $5.01(0.303)$ & $5.65(0.371)$ & $6.63(0.454)$ & $4.80(0.022)^{b}$ \\
\hline & $3^{1} A$ & $n / \pi \rightarrow \pi^{*}$ & $5.03(0.149)$ & $5.19(0.141)$ & $6.03(0.030)$ & $7.10(0.019)$ & $5.36(0.001)^{b}$ \\
\hline \multirow[t]{2}{*}{ Cyclopentadiene } & $1^{1} B_{2}$ & $\pi \rightarrow \pi^{*}$ & $4.24(0.191)$ & $4.45(0.203)$ & $5.30(0.238)$ & $6.96(0.328)$ & $5.55(0.097)$ \\
\hline & $2^{1} A_{1}$ & $\pi \rightarrow \pi^{*}$ & $6.54(0.696)$ & $6.76(0.711)$ & $7.52(0.663)$ & $8.78(0.562)$ & $6.31(0.648)$ \\
\hline \multirow[t]{2}{*}{ Cyclopropene } & $1^{1} B_{1}$ & $\sigma \rightarrow \pi^{*}$ & $7.18(0.025)$ & $7.32(0.004)$ & $7.23(0.004)$ & $7.21(0.003)$ & $6.76(0.001)$ \\
\hline & $1^{1} B_{2}$ & $\pi \rightarrow \pi^{*}$ & $6.03(0.247)$ & $6.19(0.359)$ & $6.98(0.302)$ & $7.90(0.140)$ & $7.06(0.083)$ \\
\hline \multirow[t]{2}{*}{ Cytosine } & $2^{1} A^{\prime}$ & $\pi \rightarrow \pi^{*}$ & $4.91(0.235)$ & $4.89(0.278)$ & $5.10(0.170)$ & $5.32(0.083)$ & $4.66(0.058)$ \\
\hline & $1^{1} A^{\prime \prime}$ & $n \rightarrow \pi^{*}$ & $5.54(0.023)$ & $5.42(0.001)$ & $5.58(0.000)$ & $5.80(0.000)$ & $4.87(0.002)$ \\
\hline \multirow[t]{2}{*}{ Formaldehyde } & $1^{1} A_{2}$ & $n \rightarrow \pi^{*}$ & $4.34(0.000)$ & $4.32(0.000)$ & $4.16(0.000)$ & $3.93(0.000)$ & $3.88(0.000)$ \\
\hline & $2^{1} A_{1}$ & $\pi \rightarrow \pi^{*}$ & $8.03(0.490)$ & $8.37(0.493)$ & $9.61(0.269)$ & $10.10(0.002)$ & $9.30(0.374)$ \\
\hline \multirow[t]{2}{*}{ Formamide } & $1^{1} A^{\prime \prime}$ & $n \rightarrow \pi^{*}$ & $6.80(0.003)$ & $6.99(0.004)$ & $7.83(0.004)$ & $5.92(0.005)$ & $5.63(0.001)$ \\
\hline & $2^{1} A^{\prime}$ & $\pi \rightarrow \pi^{*}$ & $7.02(0.171)$ & $7.19(0.174)$ & $8.18(0.168)$ & $9.26(0.612)$ & $7.44(0.371)$ \\
\hline \multirow[t]{2}{*}{ Imidazole } & $2^{1} A^{\prime}$ & $\pi \rightarrow \pi^{*}$ & $-{ }^{c}$ & $-{ }^{c}$ & $-{ }^{c}$ & $8.57(0.577)$ & $6.19(0.088)$ \\
\hline & $1^{1} A^{\prime \prime}$ & $n \rightarrow \pi^{*}$ & $-^{c}$ & $-^{c}$ & $-^{c}$ & $8.96(0.005)$ & $6.81(0.005)$ \\
\hline \multirow[t]{2}{*}{ Norbornadiene } & $1^{1} A_{2}$ & $\pi \rightarrow \pi^{*}$ & $4.43(0.000)$ & $4.60(0.000)$ & $5.45(0.000)$ & $7.00(0.000)$ & $5.34(0.000)$ \\
\hline & $1^{1} B_{2}$ & $\pi \rightarrow \pi^{*}$ & $5.52(0.366)$ & $5.71(0.367)$ & $6.67(0.383)$ & $8.37(0.368)$ & $6.11(0.029)$ \\
\hline \multirow[t]{2}{*}{$p$-Benzoquinone } & $1^{1} B_{1 g}$ & $n \rightarrow \pi^{*}$ & $2.31(0.000)$ & $2.78(0.000)$ & $2.96(0.000)$ & $3.96(0.000)$ & $2.78(0.000)$ \\
\hline & $1^{1} B_{1 u}$ & $\pi \rightarrow \pi^{*}$ & $4.10(1.300)$ & $4.33(1.339)$ & $5.31(1.498)$ & $7.00(1.808)$ & $5.29(0.558)$ \\
\hline \multirow[t]{2}{*}{ Propanamide } & $1^{1} A^{\prime \prime}$ & $n \rightarrow \pi^{*}$ & $-^{c}$ & $-^{c}$ & $--^{c}$ & $8.47(0.020)$ & $5.72(0.001)$ \\
\hline & $2^{1} A^{\prime}$ & $\pi \rightarrow \pi^{*}$ & $-^{c}$ & $-^{c}$ & $-^{c}$ & $10.18(0.296)$ & $7.20(0.108)$ \\
\hline \multirow[t]{2}{*}{ Pyrazine } & $1^{1} B_{3 u}$ & $n \rightarrow \pi^{*}$ & $4.04(0.011)$ & $4.08(0.010)$ & $4.47(0.011)$ & $5.19(0.012)$ & $3.95(0.008)$ \\
\hline & $1^{1} B_{2 u}$ & $\pi \rightarrow \pi^{*}$ & $5.50(0.503)$ & $5.60(0.518)$ & $5.87(0.557)$ & $6.61(0.631)$ & $4.64(0.067)$ \\
\hline \multirow[t]{2}{*}{ Pyridazine } & $1^{1} B_{1}$ & $n \rightarrow \pi^{*}$ & $3.52(0.010)$ & $3.62(0.010)$ & $4.18(0.011)$ & $5.07(0.013)$ & $3.78(0.007)$ \\
\hline & $2^{1} A_{1}$ & $\pi \rightarrow \pi^{*}$ & $5.83(0.544)$ & $5.99(0.561)$ & $6.54(0.618)$ & $7.75(0.740)$ & $5.18(0.014)$ \\
\hline Pyridine & $1^{1} B_{1}$ & $n \rightarrow \pi^{*}$ & $4.76(0.007)$ & $4.86(0.007)$ & $5.36(0.008)$ & $6.21(0.009)$ & $4.59(0.006)$ \\
\hline & $1^{1} B_{2}$ & $\pi \rightarrow \pi^{*}$ & $5.76(0.523)$ & $5.89(0.538)$ & $6.28(0.583)$ & $7.16(0.673)$ & $4.85(0.022)$ \\
\hline Pyrimidine & $1^{1} B_{1}$ & $n \rightarrow \pi^{*}$ & $4.45(0.011)$ & $4.53(0.011)$ & $5.02(0.012)$ & $5.84(0.014)$ & $4.55(0.007)$ \\
\hline & $1^{1} A_{2}$ & $n \rightarrow \pi^{*}$ & $5.80(0.000)$ & $5.91(0.000)$ & $6.41(0.000)$ & $7.26(0.000)$ & $4.91(0.000)$ \\
\hline & $1^{1} B_{2}$ & $\pi \rightarrow \pi^{*}$ & $5.97(0.548)$ & $6.13(0.566)$ & $6.67(0.625)$ & $7.88(0.751)$ & $5.44(0.022)$ \\
\hline & $2^{1} A_{1}$ & $\pi \rightarrow \pi^{*}$ & $6.40(0.520)$ & $6.64(0.531)$ & $7.47(0.563)$ & $8.96(0.628)$ & $6.95(0.038)$ \\
\hline$s$-Tetrazine & $1^{1} B_{3 u}$ & $n \rightarrow \pi^{*}$ & $2.38(0.015)$ & $2.42(0.015)$ & $2.82(0.017)$ & $3.41(0.019)$ & $2.24(0.009)$ \\
\hline & $1^{1} A_{u}$ & $\pi \rightarrow \pi^{*}$ & $5.44(0.000)$ & $5.50(0.000)$ & $5.76(0.000)$ & $6.17(0.000)$ & $3.48(0.000)$ \\
\hline Thymine & $1^{1} A^{\prime \prime}$ & $n \rightarrow \pi^{*}$ & $5.13(0.003)$ & $5.20(0.002)$ & $5.51(0.002)$ & $5.94(0.001)$ & $4.82(0.000)$ \\
\hline & $2^{1} A^{\prime}$ & $\pi \rightarrow \pi^{*}$ & $4.86(0.745)$ & $5.03(0.764)$ & $5.70(0.811)$ & $6.53(0.421)$ & $5.20(0.222)$ \\
\hline Uracil & $1^{1} A^{\prime \prime}$ & $n \rightarrow \pi^{*}$ & $5.02(0.002)$ & $5.10(0.002)$ & $5.38(0.002)$ & $5.78(0.001)$ & $4.80(0.000)$ \\
\hline & $2^{1} A^{\prime}$ & $\pi \rightarrow \pi^{*}$ & $4.92(0.693)$ & $5.11(0.718)$ & $5.80(0.793)$ & $6.69(0.356)$ & $5.35(0.224)$ \\
\hline & & MD: & $0.01^{d}$ & $0.14^{d}$ & $0.72^{d}$ & 1.72 & - \\
\hline & & MAD: & $0.58^{d}$ & $0.56^{d}$ & $0.74^{d}$ & 1.72 & - \\
\hline & & RMSD: & $0.73^{d}$ & $0.70^{d}$ & $0.92^{d}$ & 1.87 & - \\
\hline & & SD: & $0.74^{d}$ & $0.70^{d}$ & $0.57^{d}$ & 0.74 & - \\
\hline & & MAX: & $1.96^{d}$ & $2.02^{d}$ & $2.28^{d}$ & 2.98 & - \\
\hline
\end{tabular}

$a$ Best estimate energy value from Ref. S6 (CCSD value for oscillator strength). ${ }^{b}$ Structure and reference values (SCS-CC2/aug-cc-pVDZ) from Ref. S5. ${ }^{c}$ A $\sigma^{*}$ orbital is populated in the $(N+2)$ electron reference instead of the $\pi^{*}$ orbital, hence no comparison is made. ${ }^{d}$ Statistical data without acetamide, acetone, imidazole, and propanamide. 
Table S10: Vertical excitation energies computed with $h h$-TDA for different states on small molecule. Unless noted otherwise, systems are taken from Ref. S6. Different range-separated hybrid functionals are considered and the spherical def2-SV $(\mathrm{P})^{\mathrm{S} 9, \mathrm{~S} 10}$ basis set is used throughout. The values are given in $\mathrm{eV}$ (dimensionless oscillator strength in parentheses).

\begin{tabular}{|c|c|c|c|c|c|c|c|c|}
\hline system & & ate & CAM-B3LYP & $\omega$ PBEh & $\omega \mathrm{B} 97$ & $\omega \mathrm{B} 97 \mathrm{X}$ & $\omega \mathrm{B} 97 \mathrm{X}-\mathrm{D} 3$ & ref. $^{a}$ \\
\hline \multirow[t]{2}{*}{ Acetamide } & $1^{1} A^{\prime \prime}$ & $n \rightarrow \pi^{*}$ & $-^{c}$ & $-c$ & $6.79(0.018)$ & $6.64(0.018)$ & $6.52(0.018)$ & $5.80(0.001)$ \\
\hline & $2^{1} A^{\prime}$ & $\pi \rightarrow \pi^{*}$ & $-^{c}$ & $-^{c}$ & $8.42(0.439)$ & $8.27(0.413)$ & $8.12(0.401)$ & $7.27(0.223)$ \\
\hline \multirow[t]{2}{*}{ Acetone } & $1^{1} A_{2}$ & $n \rightarrow \pi^{*}$ & $-^{c}$ & $5.06(0.000)$ & $5.53(0.000)$ & $5.39(0.000)$ & $5.26(0.000)$ & $4.40(0.000)$ \\
\hline & $2^{1} A_{1}$ & $\pi \rightarrow \pi^{*}$ & ${ }^{c} c$ & $8.50(0.769)$ & $9.11(0.866)$ & $9.00(0.831)$ & $8.81(0.805)$ & $9.40(0.256)$ \\
\hline \multirow[t]{2}{*}{ Adenine } & $1^{1} A^{\prime \prime}$ & $n \rightarrow \pi^{*}$ & $5.87(0.000)$ & $5.76(0.000)$ & $6.35(0.000)$ & $6.16(0.000)$ & $6.00(0.000)$ & $5.12(0.001)$ \\
\hline & $3^{1} A^{\prime}$ & $\pi \rightarrow \pi^{*}$ & $5.46(0.933)$ & $5.45(0.933)$ & $6.02(1.037)$ & $5.84(0.997)$ & $5.69(0.971)$ & $5.25(0.297)$ \\
\hline \multirow[t]{2}{*}{ Aspirin } & $2^{1} A$ & $\pi \rightarrow \pi^{*}$ & $5.59(0.311)$ & $5.52(0.409)$ & $6.04(0.459)$ & $5.86(0.426)$ & $5.72(0.414)$ & $4.80(0.022)^{b}$ \\
\hline & $3^{1} A$ & $n / \pi \rightarrow \pi^{*}$ & $5.44(0.127)$ & $5.30(0.028)$ & $5.53(0.009)$ & $5.49(0.013)$ & $5.43(0.020)$ & $5.36(0.001)^{b}$ \\
\hline \multirow[t]{2}{*}{ Cyclopentadiene } & $1^{1} B_{2}$ & $\pi \rightarrow \pi^{*}$ & $4.78(0.225)$ & $4.68(0.222)$ & $5.16(0.262)$ & $5.02(0.245)$ & $4.88(0.235)$ & $5.55(0.097)$ \\
\hline & $2^{1} A_{1}$ & $\pi \rightarrow \pi^{*}$ & $7.22(0.695)$ & $7.13(0.713)$ & $7.78(0.687)$ & $7.54(0.690)$ & $7.36(0.698)$ & $6.31(0.648)$ \\
\hline \multirow[t]{2}{*}{ Cyclopropene } & $1^{1} B_{1}$ & $\sigma \rightarrow \pi^{*}$ & $7.33(0.004)$ & $7.35(0.004)$ & $7.49(0.003)$ & $7.38(0.003)$ & $7.34(0.003)$ & $6.76(0.001)$ \\
\hline & $1^{1} B_{2}$ & $\pi \rightarrow \pi^{*}$ & $6.37(0.347)$ & $6.26(0.351)$ & $6.55(0.332)$ & $6.48(0.335)$ & $6.40(0.342)$ & $7.06(0.083)$ \\
\hline \multirow[t]{2}{*}{ Cytosine } & $2^{1} A^{\prime}$ & $\pi \rightarrow \pi^{*}$ & $4.88(0.168)$ & $4.84(0.170)$ & $4.82(0.111)$ & $4.82(0.123)$ & $4.83(0.140)$ & $4.66(0.058)$ \\
\hline & $1^{1} A^{\prime \prime}$ & $n \rightarrow \pi^{*}$ & $5.34(0.000)$ & $5.28(0.000)$ & $5.10(0.000)$ & $5.16(0.000)$ & $5.23(0.000)$ & $4.87(0.002)$ \\
\hline \multirow[t]{2}{*}{ Formaldehyde } & $1^{1} A_{2}$ & $n \rightarrow \pi^{*}$ & $4.21(0.000)$ & $4.23(0.000)$ & $4.19(0.000)$ & $4.18(0.000)$ & $4.20(0.000)$ & $3.88(0.000)$ \\
\hline & $2^{1} A_{1}$ & $\pi \rightarrow \pi^{*}$ & $8.56(0.502)$ & $8.41(0.502)$ & $8.66(0.530)$ & $8.69(0.496)$ & $8.60(0.501)$ & $9.30(0.374)$ \\
\hline \multirow[t]{2}{*}{ Formamide } & $1^{1} A^{\prime \prime}$ & $n \rightarrow \pi^{*}$ & $7.51(0.004)$ & $6.09(0.006)$ & $6.06(0.006)$ & $6.02(0.006)$ & $6.04(0.006)$ & $5.63(0.001)$ \\
\hline & $2^{1} A^{\prime}$ & $\pi \rightarrow \pi^{*}$ & $7.58(0.178)$ & $7.77(0.548)$ & $8.00(0.580)$ & $7.96(0.564)$ & $7.89(0.556)$ & $7.44(0.371)$ \\
\hline \multirow[t]{2}{*}{ Imidazole } & $2^{1} A^{\prime}$ & $\pi \rightarrow \pi^{*}$ & $-c$ & $6.40(0.484)$ & $6.89(0.523)$ & $6.72(0.505)$ & $6.58(0.495)$ & $6.19(0.088)$ \\
\hline & $1^{1} A^{\prime \prime}$ & $n \rightarrow \pi^{*}$ & $-^{c}$ & $7.22(0.004)$ & $7.54(0.004)$ & $7.40(0.004)$ & $7.33(0.004)$ & $6.81(0.005)$ \\
\hline \multirow[t]{2}{*}{ Norbornadiene } & $1^{1} A_{2}$ & $\pi \rightarrow \pi^{*}$ & $5.01(0.000)$ & $4.86(0.000)$ & $5.45(0.000)$ & $5.26(0.000)$ & $5.09(0.000)$ & $5.34(0.000)$ \\
\hline & ${ }^{1} B_{2}$ & $\pi \rightarrow \pi^{*}$ & $6.25(0.388)$ & $6.10(0.380)$ & $6.80(0.396)$ & $6.57(0.390)$ & $6.38(0.386)$ & $6.11(0.029)$ \\
\hline \multirow[t]{2}{*}{$p$-Benzoquinone } & $1^{1} B_{1 g}$ & $n \rightarrow \pi^{*}$ & $2.52(0.000)$ & $2.33(0.000)$ & $2.59(0.000)$ & $2.50(0.000)$ & $2.44(0.000)$ & $2.78(0.000)$ \\
\hline & $1^{1} B_{1 u}$ & $\pi \rightarrow \pi^{*}$ & $4.93(1.523)$ & $4.89(1.533)$ & $5.51(1.727)$ & $5.35(1.651)$ & $5.18(1.601)$ & $5.29(0.558)$ \\
\hline \multirow[t]{2}{*}{ Propanamide } & $1^{1} A^{\prime \prime}$ & $n \rightarrow \pi^{*}$ & $6.83(0.024)$ & $-c$ & $7.12(0.022)$ & $6.99(0.023)$ & $6.85(0.023)$ & $5.72(0.001)$ \\
\hline & $2^{1} A^{\prime}$ & $\pi \rightarrow \pi^{*}$ & $8.14(0.325)$ & $-^{c}$ & $8.55(0.377)$ & $8.41(0.350)$ & $8.24(0.340)$ & $7.20(0.108)$ \\
\hline \multirow[t]{2}{*}{ Pyrazine } & $1^{1} B_{3 u}$ & $n \rightarrow \pi^{*}$ & $4.17(0.011)$ & $4.06(0.011)$ & $4.22(0.011)$ & $4.17(0.011)$ & $4.13(0.011)$ & $3.95(0.008)$ \\
\hline & $1^{1} B_{2 u}$ & $\pi \rightarrow \pi^{*}$ & $5.97(0.535)$ & $5.92(0.532)$ & $6.52(0.567)$ & $6.25(0.552)$ & $6.08(0.543)$ & $4.64(0.067)$ \\
\hline \multirow[t]{2}{*}{ Pyridazine } & $1^{1} B_{1}$ & $n \rightarrow \pi^{*}$ & $3.77(0.011)$ & $3.65(0.010)$ & $3.90(0.011)$ & $3.83(0.011)$ & $3.77(0.011)$ & $3.78(0.007)$ \\
\hline & $2^{1} A_{1}$ & $\pi \rightarrow \pi^{*}$ & $6.44(0.609)$ & $6.36(0.602)$ & $7.05(0.681)$ & $6.77(0.647)$ & $6.58(0.567)$ & $5.18(0.014)$ \\
\hline \multirow[t]{2}{*}{ Pyridine } & $1^{1} B_{1}$ & $n \rightarrow \pi^{*}$ & $4.97(0.008)$ & $4.87(0.008)$ & $5.08(0.008)$ & $5.01(0.008)$ & $4.96(0.008)$ & $4.59(0.006)$ \\
\hline & $1^{1} B_{2}$ & $\pi \rightarrow \pi^{*}$ & $6.29(0.560)$ & $6.24(0.556)$ & $6.86(0.597)$ & $6.59(0.580)$ & $6.42(0.570)$ & $4.85(0.022)$ \\
\hline \multirow[t]{4}{*}{ Pyrimidine } & $1^{1} B_{1}$ & $n \rightarrow \pi^{*}$ & $4.69(0.012)$ & $4.57(0.011)$ & $4.82(0.012)$ & $4.74(0.012)$ & $4.68(0.012)$ & $4.55(0.007)$ \\
\hline & $1^{1} A_{2}$ & $n \rightarrow \pi^{*}$ & $6.01(0.000)$ & $5.93(0.000)$ & $6.15(0.000)$ & $6.07(0.000)$ & $6.03(0.000)$ & $4.91(0.000)$ \\
\hline & $1^{1} B_{2}$ & $\pi \rightarrow \pi^{*}$ & $6.57(0.613)$ & $6.49(0.606)$ & $7.19(0.684)$ & $6.90(0.651)$ & $6.71(0.630)$ & $5.44(0.022)$ \\
\hline & $2^{1} A_{1}$ & $\pi \rightarrow \pi^{*}$ & $6.92(0.549)$ & $6.84(0.546)$ & $7.24(0.577)$ & $7.14(0.564)$ & $7.02(0.556)$ & $6.95(0.038)$ \\
\hline \multirow[t]{2}{*}{$s$-Tetrazine } & $1^{1} B_{3 u}$ & $n \rightarrow \pi^{*}$ & $2.54(0.016)$ & $2.43(0.015)$ & $2.59(0.016)$ & $2.55(0.016)$ & $2.51(0.016)$ & $2.24(0.009)$ \\
\hline & $1^{1} A_{u}$ & $\pi \rightarrow \pi^{*}$ & $5.52(0.000)$ & $5.46(0.000)$ & $5.56(0.000)$ & $5.52(0.000)$ & $5.50(0.000)$ & $3.48(0.000)$ \\
\hline \multirow[t]{2}{*}{ Thymine } & $1^{1} A^{\prime \prime}$ & $n \rightarrow \pi^{*}$ & $5.14(0.002)$ & $5.05(0.002)$ & $5.02(0.001)$ & $5.03(0.002)$ & $5.05(0.002)$ & $4.82(0.000)$ \\
\hline & $2^{1} A^{\prime}$ & $\pi \rightarrow \pi^{*}$ & $5.37(0.819)$ & $5.30(0.812)$ & $5.70(0.788)$ & $5.58(0.806)$ & $5.47(0.815)$ & $5.20(0.222)$ \\
\hline \multirow[t]{7}{*}{ Uracil } & $1^{1} A^{\prime \prime}$ & $n \rightarrow \pi^{*}$ & $5.01(0.002)$ & $4.93(0.002)$ & $4.89(0.001)$ & $4.90(0.001)$ & $4.92(0.001)$ & $4.80(0.000)$ \\
\hline & $2^{1} A^{\prime}$ & $\pi \rightarrow \pi^{*}$ & $5.46(0.796)$ & $5.39(0.791)$ & $5.84(0.862)$ & $5.70(0.838)$ & $5.58(0.820)$ & $5.35(0.224)$ \\
\hline & & MD: & $0.43^{d}$ & $0.26^{d}$ & 0.66 & 0.54 & 0.45 & - \\
\hline & & MAD: & $0.61^{d}$ & $0.53^{d}$ & 0.75 & 0.66 & 0.61 & - \\
\hline & & RMSD: & $0.80^{d}$ & $0.69^{d}$ & 0.95 & 0.84 & 0.76 & - \\
\hline & & SD: & $0.68^{d}$ & $0.65^{d}$ & 0.70 & 0.64 & 0.63 & - \\
\hline & & MAX: & $2.04^{d}$ & $1.98^{d}$ & 2.08 & 2.04 & 2.02 & - \\
\hline
\end{tabular}

${ }^{a}$ Best estimate energy value from Ref. S6 (CCSD value for oscillator strength). ${ }^{b}$ Structure and reference values (SCS-CC2/aug-cc-pVDZ) from Ref. S5. ${ }^{c}$ A $\sigma^{*}$ orbital is populated in the $(N+2)$ electron reference instead of the $\pi^{*}$ orbital, hence no comparison is made. ${ }^{d}$ Statistical data without missing data points. 

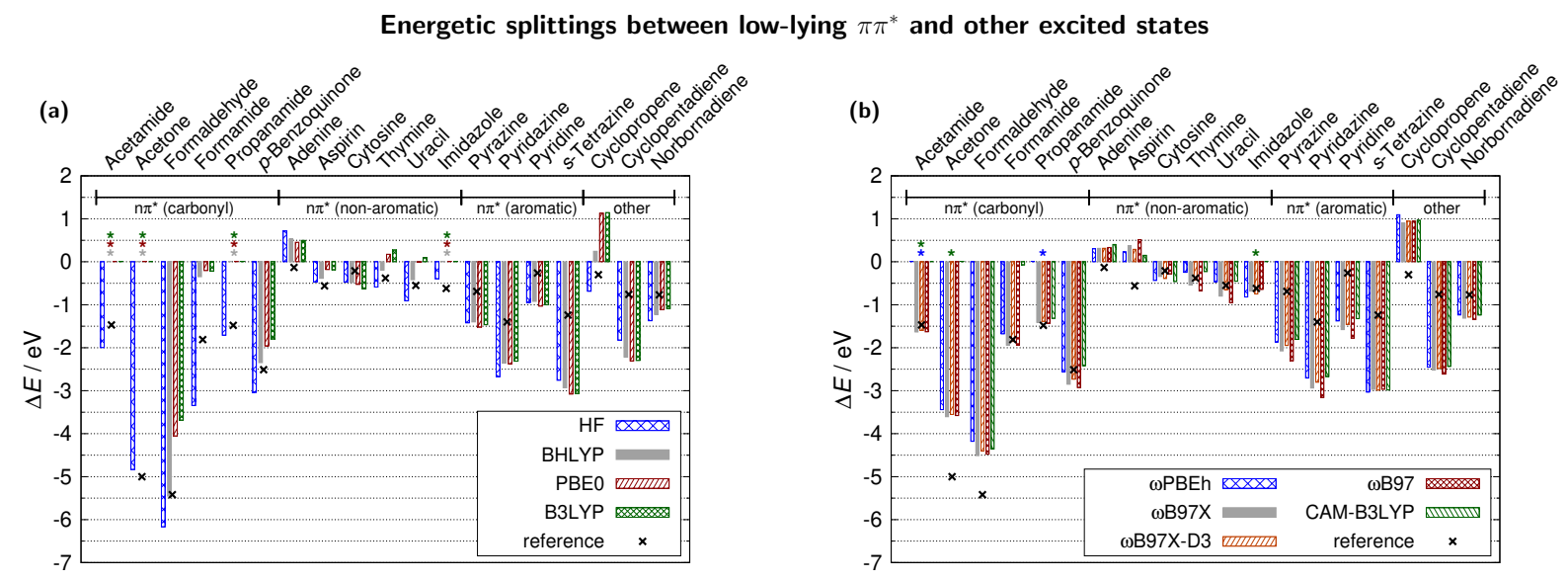

Figure S7: Excited state energy splittings for the excited states given in Tables S9 and S10 (pyrimidine, which has four states, is not included in this figure). Global hybrid functionals are grouped in a), while range-separated hybrids are in grouped in b). Entries for which no state assignment could be made, are indicated by an asterisk (see Tables S9 and S10 for details). 


\section{Basis set dependence of $h h$-TDA}

Table S11: Vertical excitation energies computed with $h h$-TDA- $\omega$ B97X with different basis sets for some small molecules. Geometries are taken from Ref. S6, while the water geometry is taken from Ref. S14. Oscillator strengths are given in parentheses. If the state ordering differs, the respective state label is also given in parentheses. The EOM-CCSD energies (for comparison) are obtained with the TZVP ${ }^{\mathrm{S} 15}$ basis set.

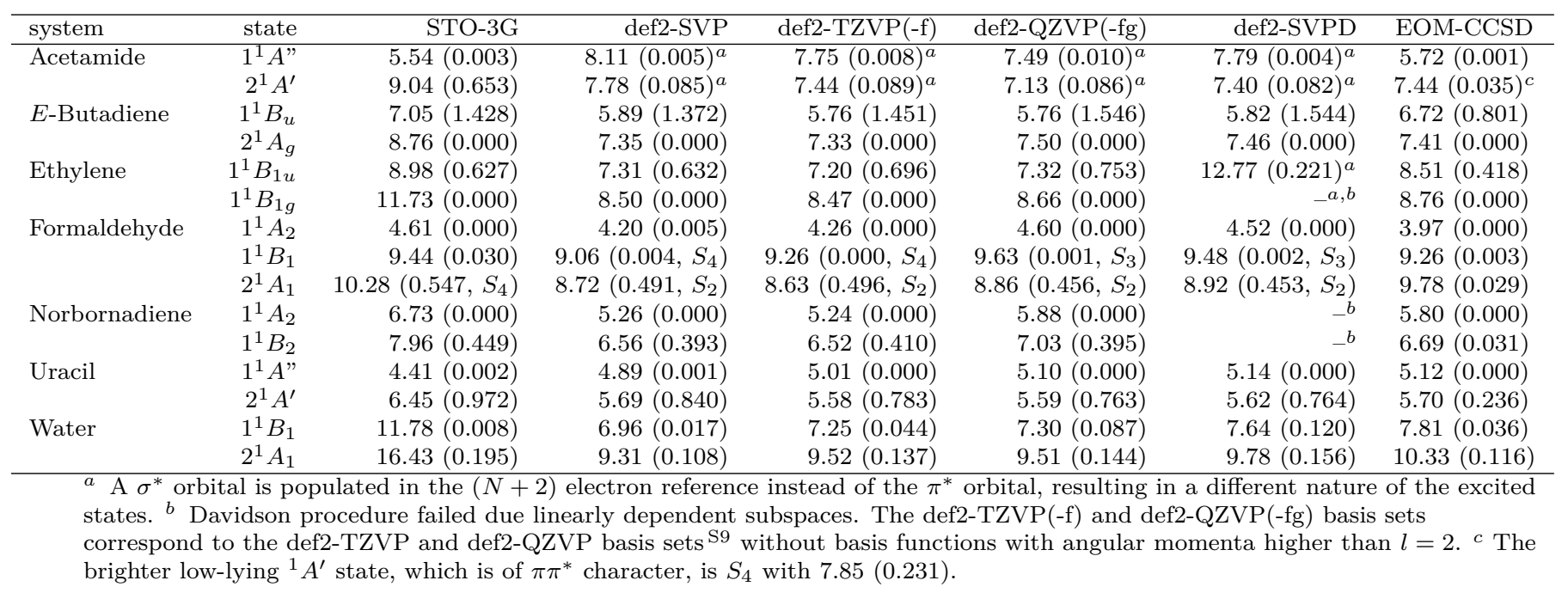

\section{Comparison of $h h$-TDA and $p p$-TDA}

Table S12: Excitation energies (in eV) computed with $h$-TDA and $p p$-TDA with the linear response (LR) type response kernel (this work) and the Hartree-Fock (HF) response kernel employed by Yang and coworkers (see main text for details). Particle-hole TDDFT (TammDancoff approximated, TDA) calculations and EOM-CCSD calculations were conducted for comparison. All calculations use the spherical TZVP ${ }^{\mathrm{S} 15}$ basis set. We chose DFAs, which we expect to perform best for both schemes, i.e., a range-separated for $h h$-TDA and a GGA for $p p$-TDA. Oscillator strengths and state ordering are denoted in parentheses. The state labels are taken from Ref. S6. ${ }^{a}$ SCF for the (N-2)-reference calculation did not converge.

\begin{tabular}{|c|c|c|c|c|c|c|c|c|}
\hline Molecule & State & Ref. & EOM-CCSD & TDA- $\omega$ B $97 X$ & $h h$-TDA- $\omega$ B97X (LR) & $h h$-TDA- $\omega$ B97X (HF) & $p p$-TDA-PBE (LR) & $p p$-TDA-PBE (HF) \\
\hline Ethene & $1 B_{1 u}(\pi \pi *)$ & 7.8 & $8.51\left(0.418, S_{1}\right)$ & $8.30\left(0.571, S_{2}\right)$ & $7.20\left(0.694, S_{1}\right)$ & $9.30\left(0.720, S_{2}\right)$ & $6.64\left(0.558, S_{1}\right)$ & $9.12\left(0.512, S_{2}\right)$ \\
\hline \multirow[t]{2}{*}{ Butadiene } & $1 B_{u}(\pi \pi *)$ & 6.18 & $6.72\left(0.801, S_{1}\right)$ & $6.44\left(1.053, S_{1}\right)$ & $5.77\left(1.452, S_{1}\right)$ & $7.07\left(1.472, S_{2}\right)$ & $4.30\left(0.969, S_{1}\right)$ & $6.39\left(1.000, S_{2}\right)$ \\
\hline & $2 A_{g}(\pi \pi *)$ & 6.55 & $7.41\left(0.000, S_{2}\right)$ & - & $7.35\left(0.000, S_{2}\right)$ & $7.05\left(0.000, S_{1}\right)$ & $7.09\left(0.000, S_{2}\right)$ & $5.98\left(0.000, S_{1}\right)$ \\
\hline \multirow[t]{2}{*}{ Hexatriene } & $1 B_{u}(\pi \pi *)$ & 5.10 & $5.72\left(1.257, S_{1}\right)$ & $5.39\left(1.613, S_{1}\right)$ & $4.93\left(2.316, S_{1}\right)$ & $5.74\left(2.204, S_{2}\right)$ & $3.22\left(1.374, S_{1}\right)$ & $4.93\left(1.436, S_{2}\right)$ \\
\hline & $2 A_{g}(\pi \pi *)$ & 5.09 & $6.61\left(0.000, S_{2}\right)$ & - & $5.95\left(0.000, S_{2}\right)$ & $5.59\left(0.000, S_{1}\right)$ & $5.64\left(0.000, S_{2}\right)$ & $4.33\left(0.000, S_{1}\right)$ \\
\hline \multirow[t]{2}{*}{ Octatetraene } & $2 A_{g}(\pi \pi *)$ & 4.47 & $5.98\left(0.000, S_{2}\right)$ & - & $5.08\left(0.000, S_{2}\right)$ & $4.77\left(0.000, S_{1}\right)$ & $4.59\left(0.000, S_{2}\right)$ & $3.37\left(0.000, S_{1}\right)$ \\
\hline & $1 B_{u}(\pi \pi *)$ & 4.66 & $5.07\left(1.727, S_{1}\right)$ & $4.72\left(2.168, S_{1}\right)$ & $4.34\left(3.192, S_{1}\right)$ & $4.89\left(2.961, S_{2}\right)$ & $2.60\left(1.773, S_{1}\right)$ & $4.09\left(1.948, S_{2}\right)$ \\
\hline Cyclopropene & $1 B_{1}(\sigma \pi *)$ & 6.76 & $6.96\left(0.087, S_{1}\right)$ & $6.65\left(0.001, S_{1}\right)$ & $7.24\left(0.003, S_{2}\right)$ & $6.93\left(0.002, S_{1}\right)$ & - & - \\
\hline
\end{tabular}




\begin{tabular}{|c|c|c|c|c|c|c|c|c|}
\hline & $1 B_{2}(\pi \pi *)$ & 7.06 & $7.24\left(0.001, S_{2}\right)$ & $6.85\left(0.122, S_{2}\right)$ & $6.35\left(0.343, S_{1}\right)$ & $7.08\left(0.152, S_{2}\right)$ & $5.72\left(0.284, S_{1}\right)$ & $7.30\left(0.262, S_{1}\right)$ \\
\hline \multirow[t]{2}{*}{ Cyclopentadiene } & $1 B_{2}(\pi \pi *)$ & 5.55 & $5.86\left(0.103, S_{1}\right)$ & $5.62\left(0.151, S_{1}\right)$ & $5.02\left(0.280, S_{1}\right)$ & $6.20\left(0.296, S_{1}\right)$ & $4.49\left(0.260, S_{1}\right)$ & $5.34\left(0.161, S_{1}\right)$ \\
\hline & $2 A_{1}(\pi \pi *)$ & 6.31 & $7.05\left(0.008, S_{2}\right)$ & $7.25\left(0.015, S_{2}\right)$ & $7.52\left(0.735, S_{2}\right)$ & $7.58\left(0.476, S_{2}\right)$ & $6.99\left(0.473, S_{2}\right)$ & $6.08\left(0.220, S_{2}\right)$ \\
\hline \multirow[t]{2}{*}{ Norbornadiene } & $1 A_{2}(\pi \pi *)$ & 5.34 & $5.80\left(0.000, S_{1}\right)$ & $5.52\left(0.000, S_{1}\right)$ & $5.24\left(0.000, S_{1}\right)$ & $6.11\left(0.000, S_{1}\right)$ & $4.61\left(0.000, S_{1}\right)$ & $5.08\left(0.000, S_{1}\right)$ \\
\hline & $1 B_{2}(\pi \pi *)$ & 6.11 & $6.69\left(0.031, S_{2}\right)$ & $6.36\left(0.035, S_{2}\right)$ & $6.53\left(0.404, S_{2}\right)$ & $7.04\left(0.307, S_{2}\right)$ & $5.81\left(0.439, S_{2}\right)$ & $6.90\left(0.364, S_{3}\right)$ \\
\hline \multirow[t]{2}{*}{ Pyridine } & $1 B_{2}(\pi \pi *)$ & 4.85 & $5.27\left(0.024, S_{2}\right)$ & $5.72\left(0.042, S_{2}\right)$ & $6.46\left(0.624, S_{2}\right)$ & $6.50\left(0.619, S_{2}\right)$ & $-^{a}$ & $-^{a}$ \\
\hline & $1 B_{1}(n \pi *)$ & 4.59 & $5.26\left(0.006, S_{1}\right)$ & $5.25\left(0.007, S_{1}\right)$ & $5.14\left(0.011, S_{1}\right)$ & $4.90\left(0.010, S_{1}\right)$ & $-^{a}$ & $-^{a}$ \\
\hline \multirow[t]{3}{*}{ Formaldehyde } & $1 A_{2}(n \pi *)$ & 3.88 & $3.97\left(0.000, S_{1}\right)$ & $3.92\left(0.000, S_{1}\right)$ & $4.26\left(0.000, S_{1}\right)$ & $3.99\left(0.000, S_{1}\right)$ & $5.25\left(0.000, S_{1}\right)$ & $3.72\left(0.000, S_{1}\right)$ \\
\hline & $1 B_{1}(\sigma \pi *)$ & 9.1 & $9.26\left(0.003, S_{2}\right)$ & $9.14\left(0.001, S_{3}\right)$ & $9.23\left(0.000, S_{4}\right)$ & $9.34\left(0.001, S_{2}\right)$ & - & - \\
\hline & $2 A_{1}(\pi \pi *)$ & 9.30 & $9.78\left(0.029, S_{3}\right)$ & $9.95\left(0.052, S_{5}\right)$ & $8.65\left(0.501, S_{2}\right)$ & $10.65\left(0.500, S_{5}\right)$ & - & - \\
\hline \multirow[t]{3}{*}{ Acetone } & $1 A_{2}(n \pi *)$ & 4.40 & $4.44\left(0.000, S_{1}\right)$ & $4.44\left(0.000, S_{1}\right)$ & $5.60\left(0.000, S_{1}\right)$ & $5.70\left(0.000, S_{1}\right)$ & $5.27\left(0.000, S_{1}\right)$ & $4.36\left(0.000, S_{1}\right)$ \\
\hline & $1 B_{1}(\sigma \pi *)$ & 9.1 & $9.26\left(0.000, S_{4}\right)$ & $9.03\left(0.001, S_{4}\right)$ & $9.96\left(0.029, S_{3}\right)$ & $10.09\left(0.067, S_{3}\right)$ & - & - \\
\hline & $2 A_{1}(\pi \pi *)$ & 9.4 & $9.13\left(0.074, S_{3}\right)$ & $9.34\left(0.182, S_{5}\right)$ & $9.06\left(0.858, S_{2}\right)$ & $10.16\left(1.009, S_{4}\right)$ & - & - \\
\hline \multirow[t]{4}{*}{ Benzoquinone } & $1 A_{u}(n \pi *)$ & 2.8 & $3.19\left(0.000, S_{2}\right)$ & $3.17\left(0.000, S_{2}\right)$ & $2.71\left(0.000, S_{2}\right)$ & $2.24\left(0.000, S_{2}\right)$ & - & - \\
\hline & $1 B_{1 g}(n \pi *)$ & 2.78 & $3.07\left(0.000, S_{1}\right)$ & $2.98\left(0.000, S_{1}\right)$ & $2.63\left(0.000, S_{1}\right)$ & $2.16\left(0.000, S_{1}\right)$ & $2.33\left(0.000, S_{1}\right)$ & $1.86\left(0.000, S_{1}\right)$ \\
\hline & $1 B_{3 g}(\pi \pi *)$ & 4.25 & $4.93\left(0.000, S_{3}\right)$ & $4.45\left(0.000, S_{3}\right)$ & $4.14\left(0.000, S_{3}\right)$ & $4.70\left(0.000, S_{4}\right)$ & - & - \\
\hline & $1 B_{1 u}(\pi \pi *)$ & 5.29 & $5.89\left(0.596, S_{4}\right)$ & $5.72\left(0.623, S_{4}\right)$ & $5.31\left(1.650, S_{5}\right)$ & $6.06\left(1.560, S_{6}\right)$ & - & - \\
\hline \multirow[t]{2}{*}{ Formamide } & $1 A "(n \pi *)$ & 5.63 & $5.66\left(0.001, S_{1}\right)$ & $5.63\left(0.001, S_{1}\right)$ & $6.12\left(0.010, S_{1}\right)$ & $5.71\left(0.006, S_{1}\right)$ & $-^{a}$ & $-^{a}$ \\
\hline & $2 A^{\prime}(\pi \pi *)$ & 7.44 & $7.52\left(0.070, S_{2}\right)$ & $7.98\left(0.086, S_{3}\right)$ & $7.79\left(0.504, S_{2}\right)$ & $7.93\left(0.502, S_{2}\right)$ & $-^{a}$ & $-^{a}$ \\
\hline \multirow[t]{2}{*}{ Acetamide } & $1 A "(n \pi *)$ & 5.8 & $5.72\left(0.001, S_{1}\right)$ & $5.71\left(0.001, S_{1}\right)$ & $6.82\left(0.011, S_{1}\right)$ & $6.74\left(0.008, S_{1}\right)$ & $-^{a}$ & $-^{a}$ \\
\hline & $2 A^{\prime}(\pi \pi *)$ & 7.27 & $7.85\left(0.231, S_{4}\right)$ & $8.29\left(0.230, S_{4}\right)$ & $8.28\left(0.405, S_{2}\right)$ & $8.29\left(0.315, S_{2}\right)$ & $-^{a}$ & $-^{a}$ \\
\hline \multirow[t]{2}{*}{ Propanamide } & $1 A^{\prime \prime}(n \pi *)$ & 5.72 & $5.74\left(0.000, S_{1}\right)$ & $5.75\left(0.001, S_{1}\right)$ & $7.13\left(0.013, S_{1}\right)$ & $7.10\left(0.010, S_{1}\right)$ & $-^{a}$ & $-{ }^{a}$ \\
\hline & $2 A^{\prime}(\pi \pi *)$ & 7.2 & $7.80\left(0.186, S_{4}\right)$ & $7.94\left(0.065, S_{3}\right)$ & $8.37\left(0.324, S_{2}\right)$ & $8.39\left(0.246, S_{2}\right)$ & $-^{a}$ & $-^{a}$ \\
\hline \multirow[t]{2}{*}{ s-Tetrazine } & $1 B_{3 u}(n \pi *)$ & 2.24 & $2.72\left(0.009, S_{1}\right)$ & $2.63\left(0.012, S_{1}\right)$ & $2.60\left(0.021, S_{1}\right)$ & $2.24\left(0.017, S_{1}\right)$ & $2.11\left(0.011, S_{1}\right)$ & $1.96\left(0.009, S_{1}\right)$ \\
\hline & $1 A_{u}(\pi \pi *)$ & 3.48 & $4.08\left(0.000, S_{2}\right)$ & $4.05\left(0.000, S_{2}\right)$ & $5.01\left(0.000, S_{2}\right)$ & $4.84\left(0.000, S_{4}\right)$ & - & - \\
\hline \multirow[t]{3}{*}{ Pyridazine } & $1 B_{1}(n \pi *)$ & 3.78 & $4.12\left(0.007, S_{1}\right)$ & $4.04\left(0.009, S_{1}\right)$ & $4.01\left(0.015, S_{1}\right)$ & $3.78\left(0.013, S_{1}\right)$ & $4.65\left(0.012, S_{1}\right)$ & $2.49\left(0.003, S_{1}\right)$ \\
\hline & $1 A_{2}(n \pi *)$ & 4.32 & $4.76\left(0.000, S_{2}\right)$ & $4.75\left(0.000, S_{2}\right)$ & - & - & $5.89\left(0.000, S_{2}\right)$ & $2.90\left(0.000, S_{2}\right)$ \\
\hline & $2 A_{1}(\pi \pi *)$ & 5.18 & $5.35\left(0.015, S_{3}\right)$ & $5.84\left(0.025, S_{3}\right)$ & $6.66\left(0.606, S_{3}\right)$ & $6.89\left(0.631, S_{3}\right)$ & - & - \\
\hline \multirow[t]{4}{*}{ Cytosine } & $2 A^{\prime}(\pi \pi *)$ & 4.66 & $4.98\left(0.062, S_{1}\right)$ & $5.33\left(0.096, S_{1}\right)$ & $4.86\left(0.135, S_{1}\right)$ & $4.18\left(0.050, S_{2}\right)$ & $-^{a}$ & $-^{a}$ \\
\hline & $1 A "(n \pi *)$ & 4.87 & $5.45\left(0.002, S_{2}\right)$ & $5.45\left(0.002, S_{2}\right)$ & $5.28\left(0.000, S_{2}\right)$ & $4.11\left(0.000, S_{1}\right)$ & $-^{a}$ & $-^{a}$ \\
\hline & $2 A "(n \pi *)$ & 5.26 & $6.00\left(0.000, S_{4}\right)$ & $6.05\left(0.000, S_{3}\right)$ & $5.68\left(0.005, S_{3}\right)$ & $4.96\left(0.005, S_{3}\right)$ & $-^{a}$ & $-^{a}$ \\
\hline & $3 A^{\prime}(\pi \pi *)$ & 5.62 & $5.95\left(0.186, S_{3}\right)$ & $6.33\left(0.163, S_{4}\right)$ & $5.88\left(0.644, S_{4}\right)$ & $5.56\left(0.555, S_{4}\right)$ & $-^{a}$ & $-^{a}$ \\
\hline \multirow[t]{4}{*}{ Thymine } & $1 A "(n \pi *)$ & 4.82 & $5.15\left(0.000, S_{1}\right)$ & $5.20\left(0.000, S_{1}\right)$ & $5.12\left(0.000, S_{1}\right)$ & $4.41\left(0.000, S_{1}\right)$ & $-^{a}$ & $-^{a}$ \\
\hline & $2 A^{\prime}(\pi \pi *)$ & 5.2 & $5.60\left(0.234, S_{2}\right)$ & $5.65\left(0.268, S_{2}\right)$ & $5.46\left(0.746, S_{2}\right)$ & $5.30\left(0.222, S_{2}\right)$ & $-^{a}$ & $-^{a}$ \\
\hline & $3 A^{\prime}(\pi \pi *)$ & 6.27 & $6.78\left(0.075, S_{4}\right)$ & $7.08\left(0.081, S_{4}\right)$ & $6.22\left(0.105, S_{3}\right)$ & $5.90\left(0.543, S_{4}\right)$ & $-^{a}$ & $-^{a}$ \\
\hline & $2 A "(n \pi *)$ & 6.16 & $6.58\left(0.000, S_{3}\right)$ & $6.53\left(0.000, S_{3}\right)$ & $6.47\left(0.000, S_{4}\right)$ & $5.38\left(0.000, S_{3}\right)$ & $-^{a}$ & $-^{a}$ \\
\hline \multirow[t]{3}{*}{ Uracil } & $1 A "(n \pi *)$ & 4.8 & $5.12\left(0.000, S_{1}\right)$ & $5.17\left(0.000, S_{1}\right)$ & $5.06\left(0.000, S_{1}\right)$ & $4.29\left(0.000, S_{1}\right)$ & $-^{a}$ & $-^{a}$ \\
\hline & $2 A^{\prime}(\pi \pi *)$ & 5.35 & $5.70\left(0.236, S_{2}\right)$ & $5.83\left(0.258, S_{2}\right)$ & $5.63\left(0.812, S_{2}\right)$ & $5.84\left(0.618, S_{4}\right)$ & $-^{a}$ & $-^{a}$ \\
\hline & $3 A^{\prime}(\pi \pi *)$ & 6.26 & $6.76\left(0.064, S_{3}\right)$ & $7.02\left(0.079, S_{4}\right)$ & $6.21\left(0.034, S_{3}\right)$ & $5.46\left(0.128, S_{3}\right)$ & $-^{a}$ & $-^{a}$ \\
\hline \multirow[t]{4}{*}{ Adenine } & $2 A^{\prime}(\pi \pi *)$ & 5.25 & $5.37\left(0.003, S_{1}\right)$ & $5.66\left(0.011, S_{2}\right)$ & - & - & $-^{a}$ & $-^{a}$ \\
\hline & $3 A^{\prime}(\pi \pi *)$ & 5.25 & $5.61\left(0.315, S_{2}\right)$ & $5.74\left(0.384, S_{3}\right)$ & $5.78\left(1.002, S_{1}\right)$ & $5.94\left(0.804, S_{2}\right)$ & $-^{a}$ & $-^{a}$ \\
\hline & $1 A "(n \pi *)$ & 5.12 & $5.58\left(0.001, S_{3}\right)$ & $5.54\left(0.001, S_{1}\right)$ & $6.22\left(0.001, S_{2}\right)$ & $5.85\left(0.001, S_{1}\right)$ & $-^{a}$ & $-^{a}$ \\
\hline & $2 A "(n \pi *)$ & 5.75 & $6.19\left(0.002, S_{4}\right)$ & $6.16\left(0.002, S_{4}\right)$ & - & - & $-^{a}$ & $-^{a}$ \\
\hline \multicolumn{3}{|c|}{ Mean deviation (MD) } & 0.43 & 0.38 & 0.36 & 0.43 & -0.25 & -0.30 \\
\hline \multicolumn{3}{|c|}{ Standard deviation (SD) } & 0.32 & 0.30 & 0.59 & 0.69 & 1.14 & 0.69 \\
\hline \multicolumn{3}{|c|}{ Mean absolute deviation (MAD) } & 0.44 & 0.40 & 0.54 & 0.67 & 0.98 & 0.59 \\
\hline \multicolumn{3}{|c|}{ Number of data points $(N)$} & 51 & 48 & 48 & 48 & 18 & 18 \\
\hline
\end{tabular}

In Table S12, we have tabulated the excitation energies computed for a subset of excitations from the Thiel benchmark set ${ }^{\mathrm{S} 6}$ computed with linear response (LR) and HF-type kernels for $h h$-TDA- $\omega$ B97X and $p p$-TDA-PBE. We note that our $p p$-TDA-PBE (HF) results are in 
agreement with the ones presented earlier by Yang and coworkers (note that we are using a slightly different basis set here). ${ }^{\text {S16 }}$ We emphasize furthermore that, due to the lack of Fock exchange, the $p p$-TDA-PBE (LR) simply corresponds to orbital energy differences at the PBE level. A significant difference between the $h h$-TDA and $p p$-TDA schemes becomes apparent when looking at systems that involve both $\pi \pi^{*}$ and other types of excitations (i.e., $n \pi^{*}$ or $\left.\sigma \pi^{*}\right)$. Here, we find that $h h$-TDA typically provides the better option, since the configuration space in $h h$-TDA is more suited to describe both excitation types simultaneously. The nucleobases and carbonyl systems are particularly good cases for $h h$-TDA, which can describe most low-lying excitation types reasonably well. For $p p$-TDA-PBE, we encounter problems for many of these systems, since the SCF of the double cation fails to converge. In the amide systems, we find that the lowest state $\left(n \pi^{*}\right)$ is properly described by $h h$-TDA as the lowest one. But the lack of higher lying virtual orbitals in $h h$-TDA results in the bright state being the second lowest state, while it is ranked as $S_{4}$ in TD-DFT and EOM-CCSD, which provide the required virtual orbital space. Regarding the choice of the response kernel, we observe that the HF-type response kernel typically leads to higher excitation energies of singly excited $\pi \pi^{*}$ states, while the other states, i.e., $n \pi^{*}, \sigma \pi^{*}$ or doubly excited $\pi \pi^{*}$ states (i.e., $2 A_{g}$ in the polyenes) are often lowered. This can improve the state splitting energies (e.g., hexatriene, octatetraene, formaldehyde, acetone), but can negatively affect the overall state ordering. This is seen in formaldehyde and acetone, where other states become lowered such that the $\pi \pi^{*}$ then corresponds to $S_{5}$ and $S_{4}$, respectively. From the series of small polyenes (butadiene through octatetraene), we see that the $h h$-TDA and $p p$ TDA schemes are able to describe the $1 B_{u}$ and $2 A_{g}$ excited states. Linear-response TDDFT, which we employ in the Tamm-Dancoff approximated scheme, cannot properly describe the latter state due to its significant double excitation character. The LR kernel variants of $h h$-TDA and $p p$-TDA lead to underestimated excitation energies for the $1 B_{u}$ state, while the $2 A_{g}$ excited states are somewhat overestimated. The latter can be rationalized by the insufficient flexibility in the orbital space, since the $2 A_{g}$ state has contributions from the 
$\mathrm{HOMO} \rightarrow(\mathrm{LUMO}+1)$ and (HOMO-1) $\rightarrow$ LUMO singly excited configurations, which cannot be described by $h h$-TDA and $p p$-TDA, respectively. As mentioned before, the use of the HFresponse kernel raises the $1 B_{u}$ excitation energies while lowering the $2 A_{g}$ state. This leads to improved energy splittings for the excitations in these polyene systems. Here, $h h$-TDA and $p p$-TDA perform equally well, with the latter showing mostly underestimated and the former mostly overestimated excitation energies compared to the best estimate reference values of Thiel and coworkers. ${ }^{S 6}$ For the different singly excited $\pi \pi^{*}$ states in cyclopentadiene and norbornadiene, the LR response kernel appears to provide better absolute excitation energies, whereas the splitting becomes better with the HF-response kernel. In summary, the best overall performance among the $p p$-RPA-derived methods is found for $h h$-TDA- $\omega$ B97X in combination with the LR-type kernel, which provides accuracy that is comparable to $p h$ TDA-DFT and is applicable to more states/systems than $p p$-TDA.

\section{State positioning of the ethylene singlet $\pi \pi^{*}$ state by the $h h$-TDA and $p p$-TDA methods}

Table S13: Description of the ethylene singlet $\pi \pi^{*}$ state with the $h h$-TDA and $p p$-TDA methods using the linear-response-type kernel (this work) and the Hartree-Fock-type kernel (Yang and coworkers) ${ }^{\mathrm{S} 16-\mathrm{S} 18}$. Three different functionals with different amounts of Fock exchange were used. All calculations were performed with the spherical def2-SV(P) ${ }^{\mathrm{S} 9, \mathrm{~S} 10}$ basis set. Energies are given in $\mathrm{eV}$, oscillator strengths given in parentheses as well as the state label indicating the positioning among the other excited states. High-level EOM-CCSD methods (excluding diffuse or Rydberg orbitals) describe this state to be the lowest excited state, i.e., as $S_{1}$ (see Table S12).

\begin{tabular}{lrrrrr}
\hline functional & amount of Fock exchange & $h h$-TDA (LR) & $h h$-TDA (HF) & $p p$-TDA (LR) & $p p$-TDA (HF) \\
\hline B3LYP & $20 \%$ & $6.32\left(0.563, \boldsymbol{S}_{\mathbf{1}}\right)$ & $9.47\left(0.565, \boldsymbol{S}_{\mathbf{2}}\right)$ & $7.39\left(0.583, \boldsymbol{S}_{\mathbf{1}}\right)$ & $9.32\left(0.529, \boldsymbol{S}_{\mathbf{3}}\right)$ \\
BHLYP & $50 \%$ & $7.94\left(0.622, \boldsymbol{S}_{\mathbf{1}}\right)$ & $9.95\left(0.621, \boldsymbol{S}_{\mathbf{2}}\right)$ & $8.16\left(0.599, \boldsymbol{S}_{\mathbf{1}}\right)$ & $9.11\left(0.555, \boldsymbol{S}_{\mathbf{4}}\right)$ \\
HF & $100 \%$ & $10.69\left(0.714, \boldsymbol{S}_{\mathbf{2}}\right)$ & $10.69\left(0.714, \boldsymbol{S}_{\mathbf{2}}\right)$ & $8.80\left(0.589, \boldsymbol{S}_{\mathbf{4}}\right)$ & $8.80\left(0.589, \boldsymbol{S}_{\mathbf{4}}\right)$ \\
\hline
\end{tabular}


(a)

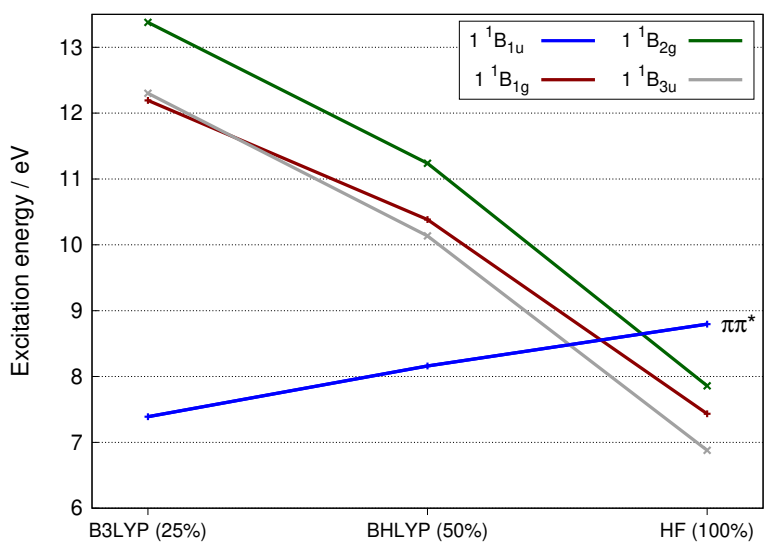

(c)

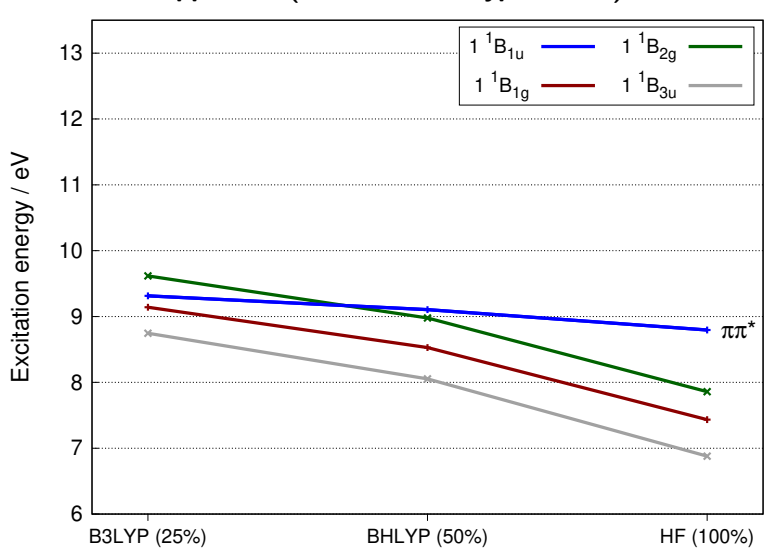

(b)

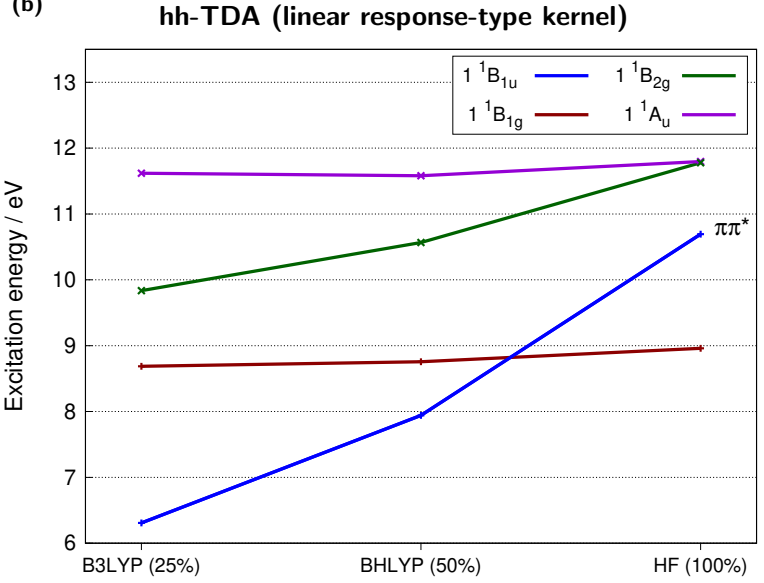

(d)

hh-TDA (Hartree-Fock-type kernel)

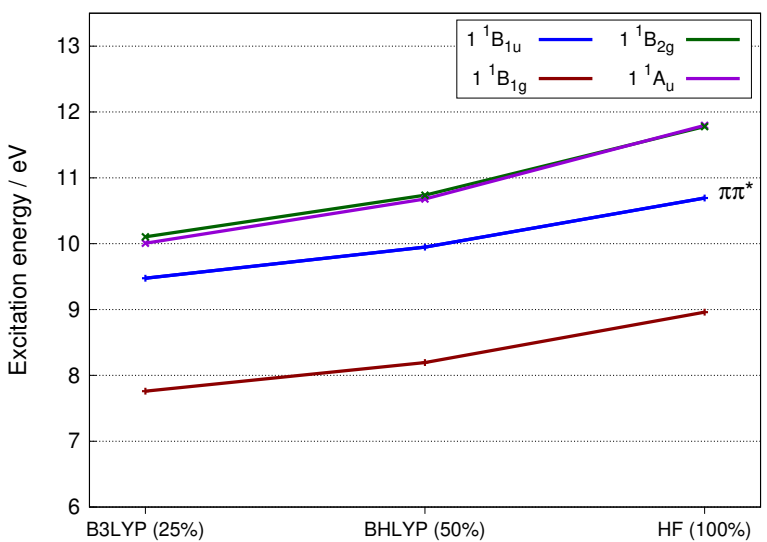

Figure S8: State energies of the four lowest excited singlet states of ethylene computed with $p p$-TDA ((a) and (c)) and $h h$-TDA ((b) and (d)) with the Hartree-Fock-type kernel of Yang and coworkers ${ }^{\mathrm{S} 16-\mathrm{S} 18}((\mathrm{c})$ and $(\mathrm{d}))$ and the linear-response-type kernel ((a) and (b), this work). Three different functionals with different amounts of Fock exchange (in parentheses) were used. All calculations were performed with the spherical def2-SV $(\mathrm{P})^{\mathrm{S} 9, \mathrm{~S} 10}$ basis set. The $1{ }^{1} \mathrm{~B}_{1 u}\left(\pi \pi^{*}\right)$ state is shown in blue and also labeled in each plot.

\section{Twisted-ethylene pyramidalization potential energy}

\section{curve}

We investigated the potential energy surfaces of the ground and first excited state of ethylene along the torsion and pyramidalization coordinates (see main text for details and results). 
hh-TDA-wB97X with a Cartesian def2-SVP basis was used for this purpose. In Figure S9, we show the potential energy curves for a rigid scan along the pyramidalization angle at a fixed torsion of 90 degrees. In addition to $h h$-TDA, we have computed energies with 3-stateaveraged, extended multi-state complete active space second-order perturbation method with a $(2,2)$ active space (abbreviated as SA3-XMS-CAS(2,2)PT2 in the following). The leastsquares tensor hypercontraction (LS-THC) ${ }^{\text {S19 }}$ scheme along with the cc-pVDZ density fitting basis set ${ }^{\mathrm{S} 20}$ was used to accelerate the computation of two-electron integrals. 


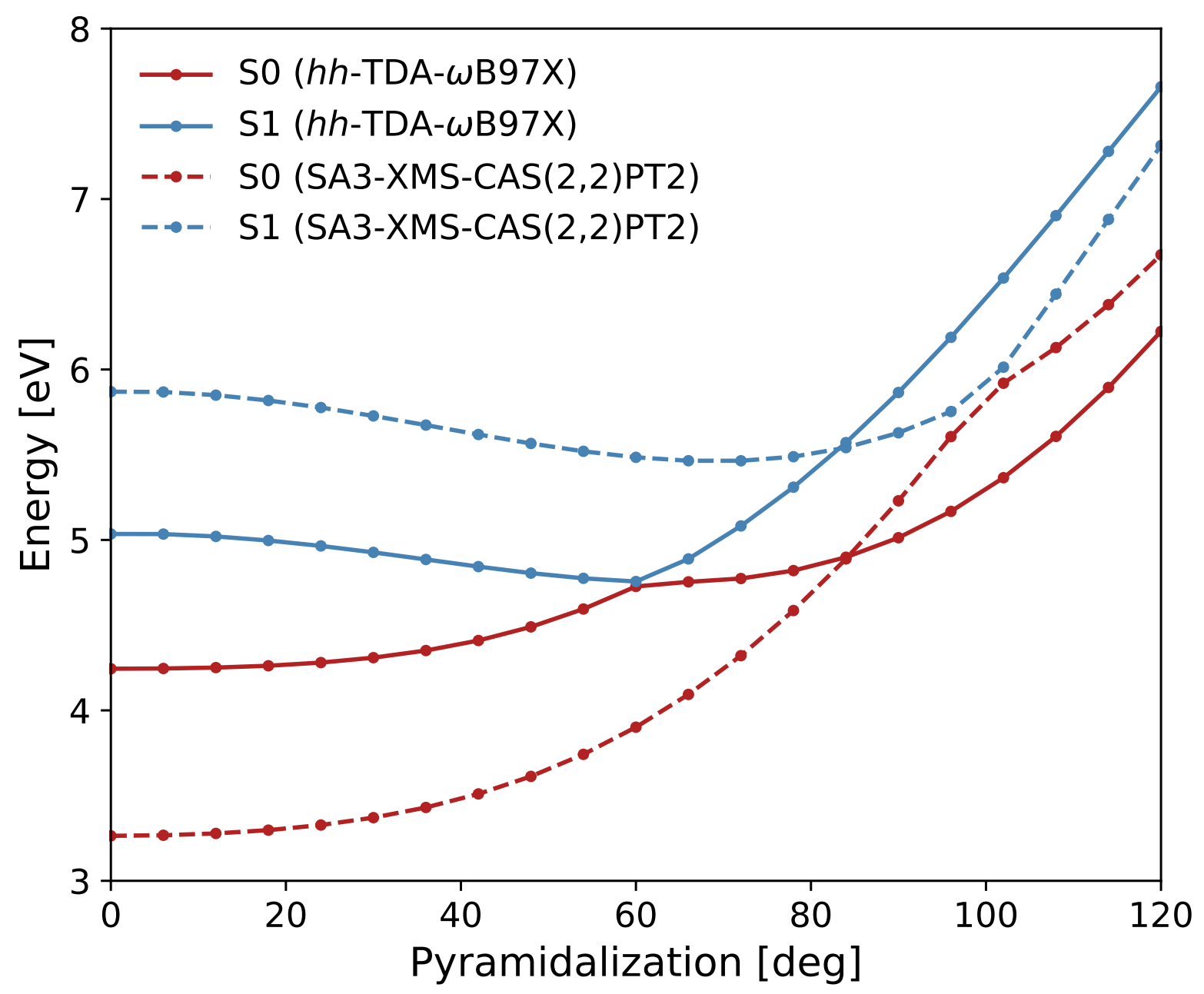

Figure S9: Potential energy curves for the $S_{1}$ (blue) and $S 0$ (red) states of ethylene along the pyramidalization coordinate at fixed 90 degree torsion computed by $h h$-TDA- $\omega$ B97X (solid lines) SA3-XMS-CAS(2,2)PT2 ${ }^{\mathrm{S} 21}$ (dashed lines). The Cartesian def2-SVP ${ }^{\mathrm{S} 9, \mathrm{~S} 10}$ basis set was used for both. This accompanies Figure 5 from the main text, where the full $h h$ TDA- $\omega$ B97X/def2-SVP potential energy surface computed by for ethylene is shown in the torsion and pyramidalization coordinates. All energies are given relative to the $S_{0}$ energy at the Franck-Condon point.

In Figure S9, it is observed that the SA3-XMS-CAS(2,2)PT2 and $h h$-TDA- $\omega$ B97X potential energy curves are in reasonably good agreement. In particular, comparison of the states in a diabatic picture reveals quite some parallelity between the two levels of theory. Compared to SA3-XMS-CAS $(2,2) \mathrm{PT} 2, h h$-TDA- $\omega \mathrm{B} 97 \mathrm{X}$ produces underestimated energies for the openshell $\pi \pi^{*}$ and overestimated energies for the closed-shell $\pi \pi$ state, which in summary results 
in a conical intersection at smaller pyramidalization angles. Nevertheless, the shapes of the both states are well-reproduced by $h h$-TDA.

\section{References}

(S1) Shao, Y.; Head-Gordon, M.; Krylov, A. I. The spin-flip approach within timedependent density functional theory: Theory and applications to diradicals. J. Chem. Phys. 2003, 118, 4807-4818.

(S2) Jacquemin, D.; Wathelet, V.; Perpète, E. A.; Adamo, C. Extensive TD-DFT Benchmark: Singlet-Excited States of Organic Molecules. J. Chem. Theory Comput. 2009, 5, 2420-2435.

(S3) Dreuw, A.; Head-Gordon, M. Single-Reference ab Initio Methods for the Calculation of Excited States of Large Molecules. Chem. Rev. 2005, 105, 4009-4037.

(S4) Risthaus, T.; Hansen, A.; Grimme, S. Excited States Using the Simplified TammDancoff-Approach for Range-Separated Hybrid Density Functionals: Development and Application. Phys. Chem. Chem. Phys. 2014, 28, 14408-14419.

(S5) Grimme, S.; Bannwarth, C. Ultra-fast computation of electronic spectra for large systems by tight-binding based simplified Tamm-Dancoff approximation (sTDA-xTB). J. Chem. Phys. 2016, 145, 054103.

(S6) Schreiber, M.; Silva-Junior, M. R.; Sauer, S. P. A.; Thiel, W. Benchmarks for electronically excited states: CASPT2, CC2, CCSD, and CC3. J. Chem. Phys. 2008, 128, 134110.

(S7) Ufimtsev, I. S.; Martínez, T. J. Quantum Chemistry on Graphical Processing Units. 2. Direct Self-Consistent-Field Implementation. J. Chem. Theory Comput. 2009, 5, $1004-1015$. 
(S8) Ufimtsev, I. S.; Martínez, T. J. Quantum Chemistry on Graphical Processing Units. 1. Strategies for Two-Electron Integral Evaluation. J. Chem. Theory Comput. 2008, 4, $222-231$.

(S9) Weigend, F.; Ahlrichs, R. Balanced basis sets of split valence, triple zeta valence and quadruple zeta valence quality for $\mathrm{H}$ to Rn: Design and assessment of accuracy. Phys. Chem. Chem. Phys. 2005, 7, 3297-3305.

(S10) Schäfer, A.; Horn, H.; Ahlrichs, R. Fully optimized contracted Gaussian basis sets for atoms Li to Kr. J. Chem. Phys. 1992, 97, 2571-2577.

(S11) Furche, F.; Ahlrichs, R.; Hättig, C.; Klopper, W.; Sierka, M.; Weigend, F. Turbomole. WIREs Comput. Mol. Sci. 2014, 4, 91-100.

(S12) Ahlrichs, R.; Bär, M.; Häser, M.; Horn, H.; Kölmel, C. Electronic Structure Calculations on Workstation Computers: The Program System Turbomole. Chem. Phys. Lett. 1989, 162, 165-169.

(S13) TURBOMOLE V6.4 2012, a development of University of Karlsruhe and Forschungszentrum Karlsruhe GmbH, 1989-2007, TURBOMOLE GmbH, since 2007; available from http: //www . turbomole.com.

(S14) Jurečka, P.; Šponer, J.; Cerny, J.; Hobza, P. Benchmark database of accurate (MP2 and $\operatorname{CCSD}(\mathrm{T})$ complete basis set limit) interaction energies of small model complexes, DNA base pairs, and amino acid pairs. Phys. Chem. Chem. Phys. 2006, 8, 1985-1993.

(S15) Schäfer, A.; Huber, C.; Ahlrichs, R. Fully optimized contracted Gaussian basis sets of triple zeta valence quality for atoms Li to Kr. J. Chem. Phys. 1994, 100, 5829-5835.

(S16) Yang, Y.; Peng, D.; Lu, J.; Yang, W. Excitation energies from particle-particle random 
phase approximation: Davidson algorithm and benchmark studies. J. Chem. Phys. 2014, 141, 124104.

(S17) Yang, Y.; van Aggelen, H.; Yang, W. Double, Rydberg and charge transfer excitations from pairing matrix fluctuation and particle-particle random phase approximation. $J$. Chem. Phys. 2013, 139, 224105.

(S18) Peng, D.; van Aggelen, H.; Yang, Y.; Yang, W. Linear-response time-dependent density-functional theory with pairing fields. J. Chem. Phys. 2014, 140, 18A522.

(S19) Hohenstein, E. G.; Parrish, R. M.; Sherrill, C. D.; Martínez, T. J. Communication: Tensor hypercontraction. III. Least-squares tensor hypercontraction for the determination of correlated wavefunctions. J. Chem. Phys. 2012, 137, 221101.

(S20) Weigend, F.; Köhn, A.; Hättig, C. Efficient use of the correlation consistent basis sets in resolution of the identity MP2 calculations. J. Chem. Phys. 2002, 116, 3175-3183.

(S21) Song, C.; Martínez, T. J. Reduced scaling CASPT2 using supporting subspaces and tensor hyper-contraction. J. Chem. Phys. 2018, 149, 044108. 